\title{
Effect of Radiation on Leukocytes Migration and Gene Expression of Rat Liver Chemokines: In-vivo and In-vitro Studies
}

\author{
Dissertation \\ zur Erlangung des Doktorgrades \\ der Mathematisch-Naturwissenschaftlichen Fakultäten \\ der Georg-August-Universität zu Göttingen
}

vorgelegt von

Ihtzaz Ahmed Malik

aus Lahore, Pakistan

Göttingen 2009

Wegen schwerwiegender Mängel bezüglich der Nutzung wissenschaftlicher Quellen zurückgezogen am 01.10.2015 Nach grundlegender Überarbeitung erneut vorgelegt am 23.02.2016 
D7

Referent: Prof. Dr. Michael Kessel

Korreferent: Prof. Dr. Uwe Groß

Tag der mündlichen Prüfung: 18.01.2010 


\section{Table of Contents}

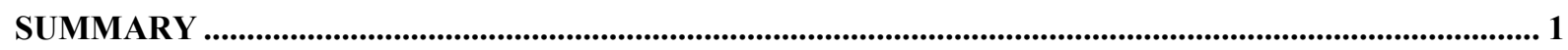

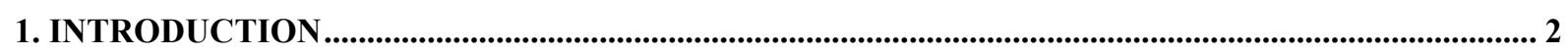

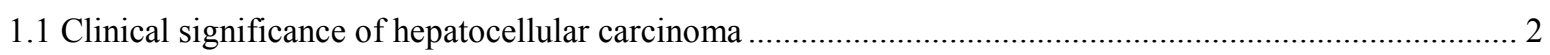

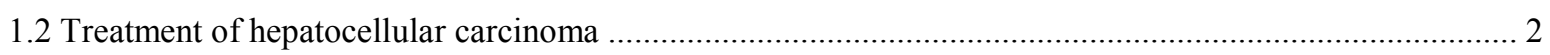

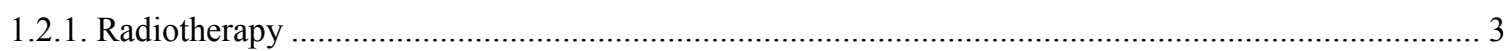

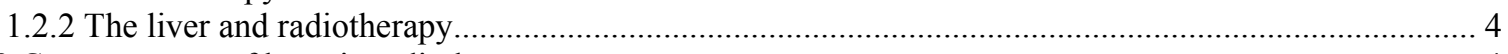

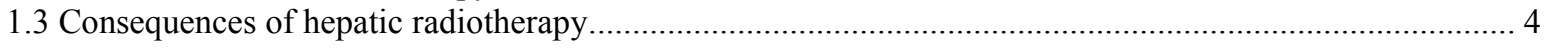

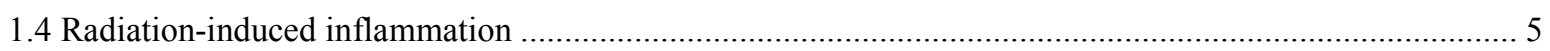

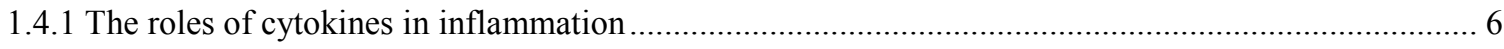

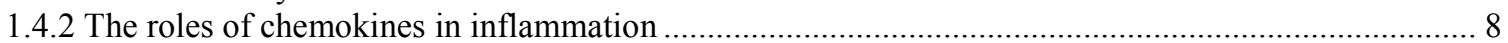

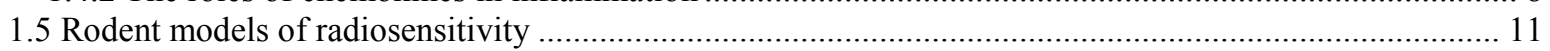

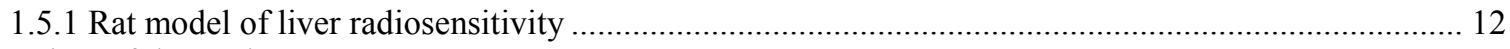

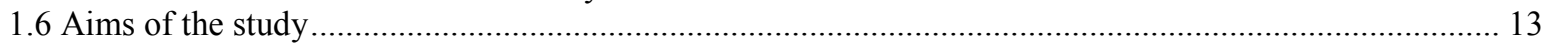

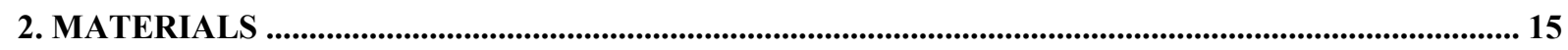

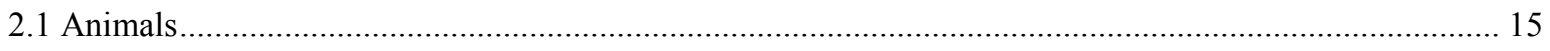

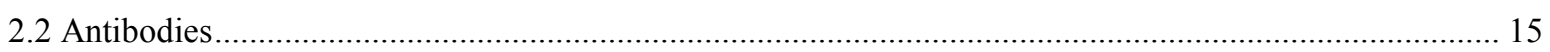

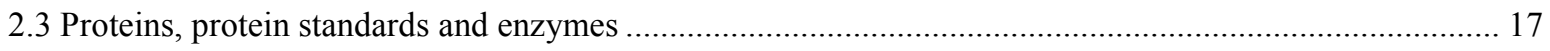

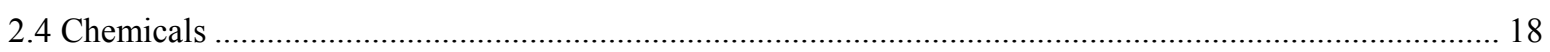

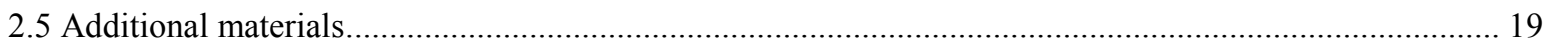

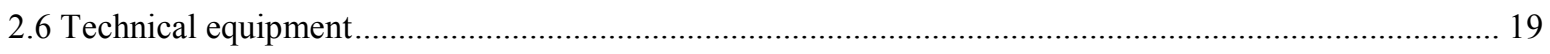

3. METHODS ………......................................................................................................................................... 19

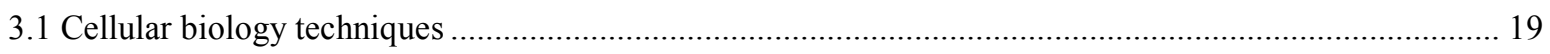

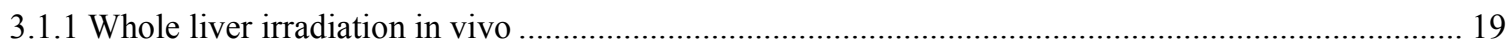

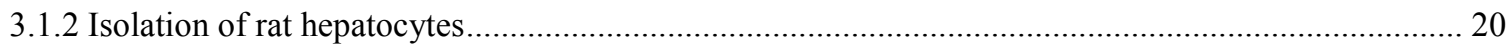

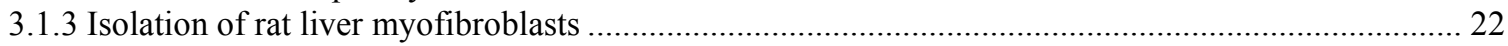

3.1.4 Primary culture of hepatic cells: treatment and harvesting ............................................................. 24

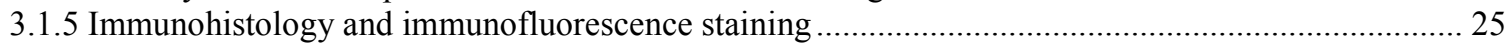

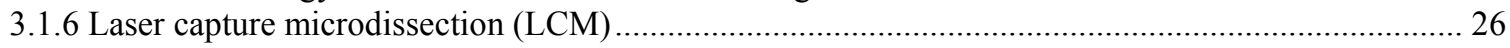

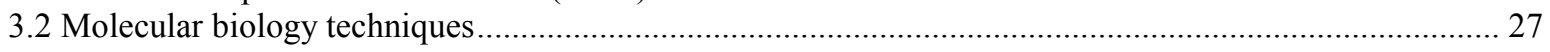

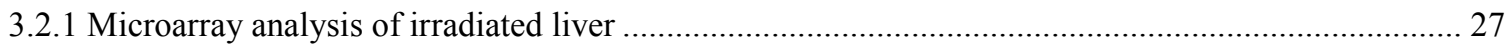

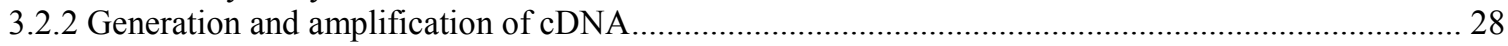

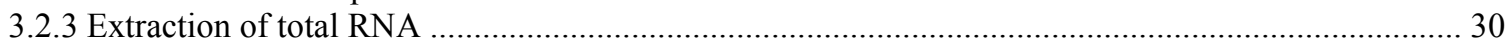

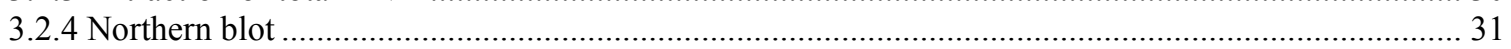

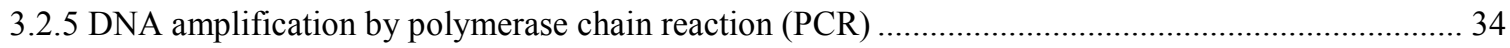

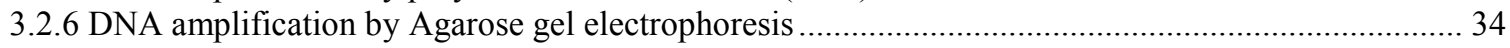

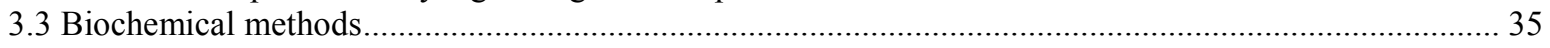

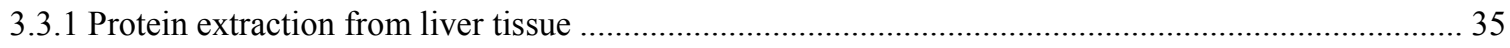

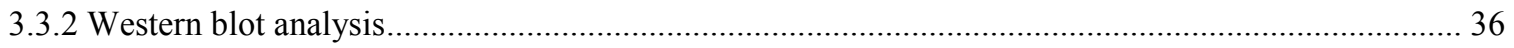

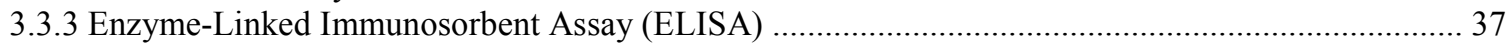

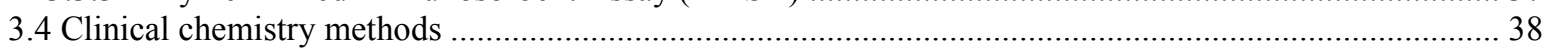

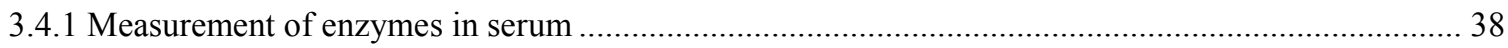

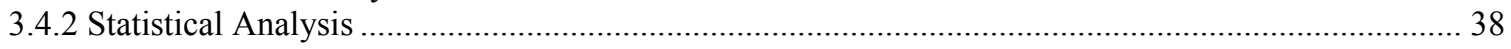

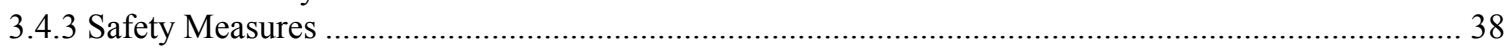

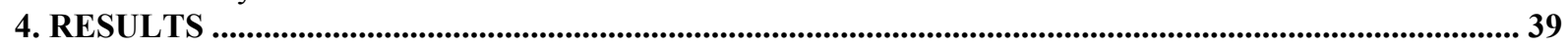

4.1 The effect of radiation on the serum levels of liver enzymes and macrophage inflammatory protein2/CXCL2 
4.2 Immunohistochemical detection of leukocytes in the irradiated rat liver ...........................................40

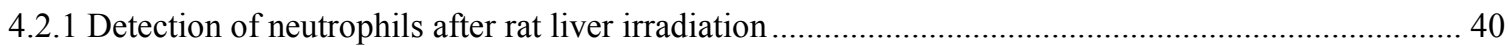

4.2.2 Identification of the recruited neutrophils and macrophages using an immunofluorescence double-

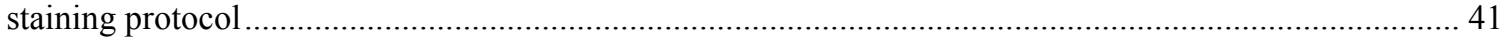

4.2.3 Relationship between neutrophils and liver myofibroblasts ................................................... 44

4.3 Immunohistochemical detection of monocyte chemoattractant protein-1/CCL2 in the irradiated rat liver 46

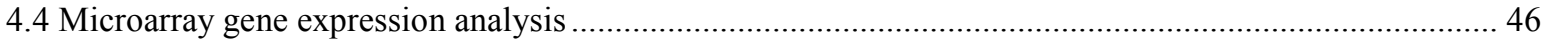

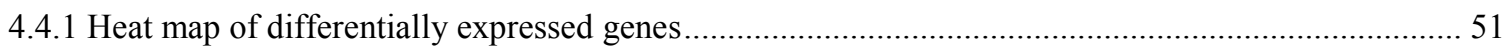

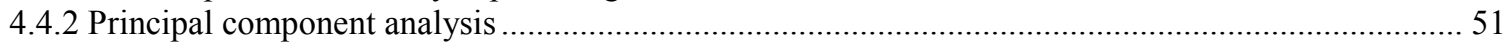

4.5 Chemokine and chemokine-receptor gene expression in irradiated rat livers.......................................5 53

4.6 Changes in chemokine gene expression in microdissected irradiated rat liver sections ........................... 55

4.7 Influence of an antibody against monocyte chemoattractant protein-1/CCL2 on hepatic gene expression

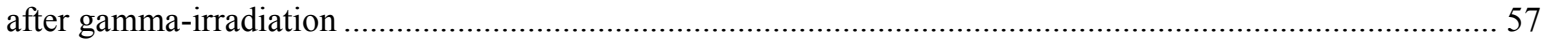

4.8 Changes in chemokine and cytokine gene expression in the parenchymal and non-parenchymal cells of

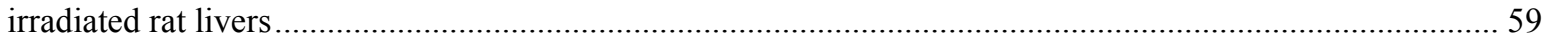

4.8.1 Alterations in chemokine gene-expression in the hepatocytes post-gamma irradiation ....................59

4.8.2 Changes in chemokine and cytokine gene expression in the liver myofibroblasts after gamma-

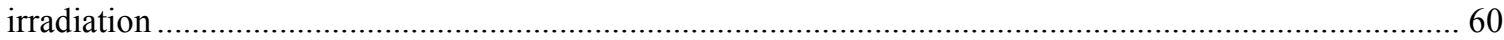

4.9 Comparisons of the chemokines' and cytokines' cycle threshold values in the liver and in the isolated cells

5. DISCUSSION

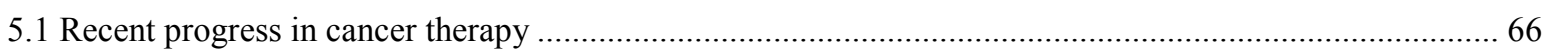

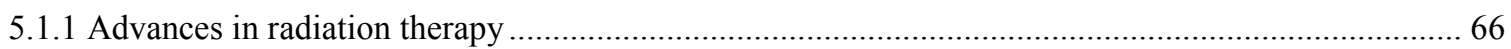

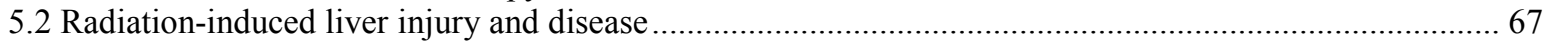

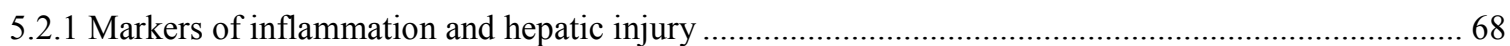

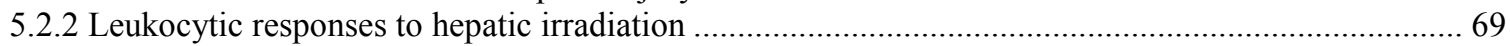

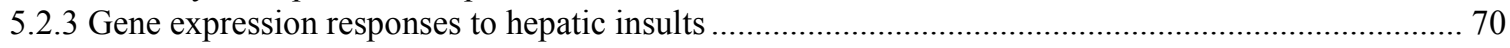

5.3 Roles of inflammatory mediators and of endothelial cells in inflammatory cell transmigration ............... 71

5.4 Roles of the chemokines and the liver myofibroblasts in inflammatory cell recruitment........................ 71

5.5 Post-irradiation reactive oxygen species production and hypoxia ................................................... 73

5.6 The tumour microenvironment and the impact of radiotherapy …............................................... 75

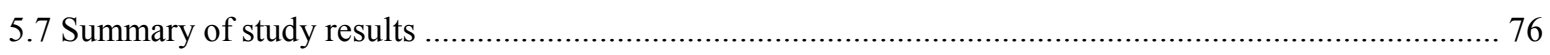

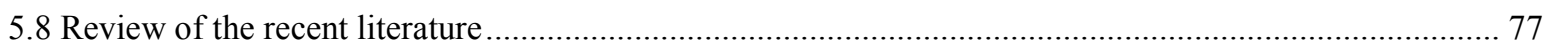

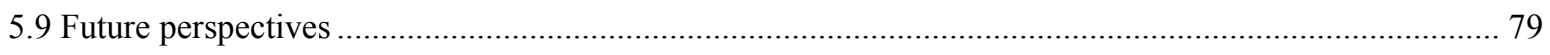

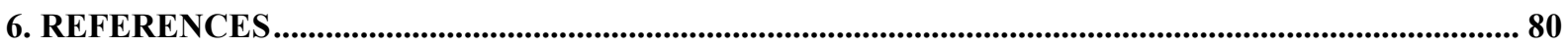

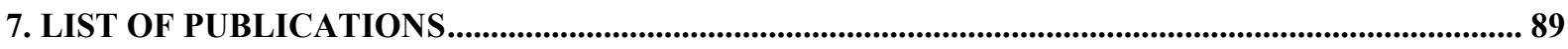

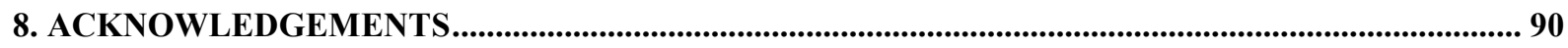

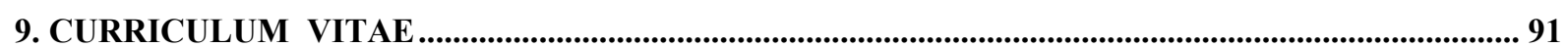




\section{LIST OF FIGURES}

Figure 1: Deoxyribonucleic acid damage caused by ionising radiation (based on suggestion by Morgan and Sowa 2005)

Figure 2: Anatomical and histological images of a normal and a fibrotic liver. (a) A normal liver, (b) a histological section of a normal liver, (c) a fibrotic liver and (d) a histological section of a fibrotic liver. ........... 5 Figure 3: The sequence of the cellular events in the transmigration process (according to Petri et al. 2008, modified and adapted to liver tissue).

Figure 4: Classification of the main $\mathrm{CXC}$ and $\mathrm{CC}$-chemokines and chemokines receptors (modified from Proudfoot 2002).

Figure 5: Serum concentrations of liver enzymes and of macrophage inflammatory protein-2 (MIP-2)/CXCL2 in rats following liver irradiation. (a) The serum alkaline phosphatase level rose 1 hour after irradiation and it declined thereafter. (b) The serum alanine aminotransferase level was slightly elevated for up to 24 hours after irradiation, while the serum aspartate aminotransferase (AST) level increased continuously for up to 24 hours after irradiation. The serum AST level increased significantly to its maximum level 24 hours after irradiation in comparison with control rats. (c) Serum levels of the MIP-2/CXCL2 protein in the irradiated and control rats. The serum concentration of MIP-2/CXCL2 was measured using an enzyme-linked immunosorbent assay. The increase in the serum concentration of MIP-2/CXCL2 between 1 hour and 3 hours after irradiation was statistically significant. Results are representative of 3 -experiments. A value of $P<0.05$ was considered statistically significant. The data presented are the means \pm standard errors of the means...... 39

Figure 6: Indirect immunodetection of neutrophil elastase (NE) in sections of (a) sham-irradiated control rat liver and (b) rat liver 6 hours after irradiation (the arrows indicate the $\mathrm{NE}^{+}$granulocytes in the portal field) (original magnifications $\times 200$; the scale bars represent $100 \mu \mathrm{m}$ ). (c) $\mathrm{NE}^{+}$cells were counted in the portal fields of view $(\mathrm{N}=10)$ at different time points following rat liver irradiation. (d) Changes in the levels of hepatic NE messenger ribonucleic acid (mRNA) expression at the different time points following rat liver irradiation, which were determined by utilising the reverse transcriptase-polymerase chain reaction. The upregulation of $\mathrm{NE}$ mRNA was detected at 1 hour after irradiation and it peaked at 6 hours after irradiation, after which it declined $(\mathrm{N}=3)$. Abbreviations: NE, neutrophil elastase; cont, control.

Figure 7: Double immunofluorescence staining of rat hepatic slices using monoclonal antibodies against ED1 (red) and rat cluster of differentiation (CD) $11 \mathrm{~b} / \mathrm{c}$ (green) after irradiation. The images on the left correspond to the livers from the sham-irradiated animals and the images on the right correspond to the livers from the irradiated animals at 6 hours after irradiation. The yellow arrow indicates the $\mathrm{ED} 1{ }^{+} \mathrm{CD} 11 \mathrm{~b} / \mathrm{c}^{+}$resident macrophages, the white arrow indicates the recruited $\mathrm{CD} 11 \mathrm{~b} / \mathrm{c}^{+} \mathrm{ED} 1^{-}$neutrophils. The images at the top of the panel show double immunofluorescence staining for CD11b/c and ED1, the images in the middle of the panel show CD11b/c staining and the images at the bottom of the panel show ED1 staining and the arrowheads indicate the portal area. The results represent the mean values from three rats and 6-slides for each time-point (original magnifications $\times 200$; scale bars represent $100 \mu \mathrm{m}$ ). Abbreviations: CD, cluster of differentiation; DAPI, 4',6-diamidino-2-phenylindole. ... 42

Figure 8: $\mathrm{ED}^{+}$and $\mathrm{ED} 2^{+}$cells in rat livers after irradiation. (a) $\mathrm{ED} 1^{+}$cells in a sham-irradiated liver, (b) $\mathrm{ED} 1^{+}$ cells in a liver 6 hours after irradiation, (c) ED2 ${ }^{+}$cells in a sham-irradiated liver and (d) ED2 ${ }^{+}$cells in a liver 6 hours after irradiation (original magnifications $\times 100$; the scale bars represent $100 \mu \mathrm{m}$ ). (e) ED $1^{+}$cells and (f) $\mathrm{ED}^{+}$cells were counted in the areas around the portal vessels at the different time points after rat liver irradiation. The results represent the mean values from three animals and six slides per time point. Abbreviation: Co, control.

Figure 9: Double immunofluorescence staining of rat hepatic slices with monoclonal antibodies against smooth muscle actin (SMA) (red) and cluster of differentiation (CD)11b/c (green) (original magnifications $\times 200$; the scale bars represent $100 \mu \mathrm{m}$ ). (a) The images on the left correspond to the livers from the sham-irradiated animals and the images on the right correspond to the livers from the irradiated animals at 6 hours after irradiation. The white arrows indicate the recruited neutrophils that were present around the portal vessels. The images at the top of the panel show double immunofluorescence staining for CD11b/c and SMA, the images in 
the middle of the panel show CD11b/c staining and the images at the bottom of the panel show SMA staining. (b) The $\mathrm{CD} 11 \mathrm{~b} / \mathrm{c}^{+}$cells were counted in the portal fields of view $(\mathrm{N}=10)$ at different time points after rat liver irradiation $(\mathrm{N}=3)$. Abbreviations: $\mathrm{CD}$, cluster of differentiation; DAPI, 4',6-diamidino-2-phenylindole; SMA, smooth muscle actin. 44

Figure 10: Double immunofluorescence staining of rat liver sections with monoclonal antibodies against Thy-1 (red) and cluster of differentiation (CD) $11 \mathrm{~b} / \mathrm{c}$ (green). The images on the left correspond to the livers from the sham-irradiated animals and the images on the right correspond to the livers from the irradiated animals at 6 hours after irradiation. The images at the top of the panel show double immunofluorescence staining for CD11b/c and Thy-1, the images in the middle of the panel show CD11b/c staining and the images at the bottom of the panel show Thy-1 staining. The upper right image shows the periportal location of the neutrophils between and around the liver myofibroblasts (LMFs) $\left(\right.$ Thy $\left.-1^{+}\right)$. The white arrow indicates the recruited neutrophils that were present around the portal vessels and the white arrow head indicates the LMFs that appear to be closely associated with the neutrophils (original magnifications $\times 200$; the scale bars represent $100 \mu \mathrm{m}$ ). The results represent the mean values from three animals and six slides per time point. Abbreviations: $C D$, cluster of differentiation; DAPI, 4',6-diamidino-2-phenylindole. 45

Figure 11: Immunodetection of monocyte chemoattractant protein (MCP)-1/CCL2 using a goat polyclonal antiserum against MCP-1/CCL2 followed by peroxidase staining in (a) sham-irradiated control rat liver sections and (b) rat liver sections 3 hours after irradiation. Compared with the livers from the sham-irradiated control animals, an increase intensity of MCP-1/CCL2 was observed within the walls of the portal vessels and in the cells accumulating around the vessels (black arrow) that peaked at 3 hours after irradiation (original magnifications $\times 200$; the scale bars represent $100 \mu \mathrm{m}$ ). (c) Negative control staining comprised the use of the secondary antibody and peroxidase staining (original magnification $\times 100$; the scale bar represents $100 \mu \mathrm{m}$ ). (d) Numbers of MCP-1/CCL2 ${ }^{+}$cells in and around the portal field $(\mathrm{N}=10)$. The results represent mean values from three animals and six slides per time point. Abbreviation: Co, control.

Figure 12: Heat map analysis of the microarray data. Gene-upregulation and -downregulation is shown in red and blue, respectively, in the corresponding sample columns. The liver samples from the control animals $(\mathrm{C} 1, \mathrm{C} 2$ and C3) were compared with those from the irradiated animals (Irr1, Irr2 and Irr3) 6 hours after irradiation. .... 52 Figure 13: Principal component analysis (PCA) of the microarray data. The PCA represents the variability among the irradiated and the control samples. The control samples are presented in blue and the irradiated samples are presented in red. The liver samples from the control animals (C1, C2 and C3) were compared with those from the irradiated animals (Irr1, Irr2 and Irr3) 6 hours after irradiation.

Figure 14: Alterations in messenger ribonucleic acid (mRNA) and protein-levels of the genes for CXC chemokines in rat livers. (a) Alterations in mRNA-level for the CXCL1, CXCL2 and CXCL5 chemokines and for CXCR2 in the irradiated livers at the different time points relative to the sham-irradiated control rats determined using the reverse transcriptase-polymerase chain reaction (RT-PCR). (b) Confirmation by agarose gel electrophoresis. Normalisation of the RT-PCR was achieved using the housekeeping genes, namely, betaactin and ubiquitin, and the control value was set at one. (c) Northern blot analysis and (d) Western blot analysis using an antibody against CXCL1. Results represent the means \pm standard errors of the means from 3experiments that were performed in duplicate and were compared with those from the sham-irradiated control rats at each time point $\left({ }^{*} P<0.05\right.$, based on a one-way analysis of variance). 54

Figure 15: Changes in the levels of messenger ribonucleic acid expression for $\mathrm{CC}$ chemokines in the irradiated rat liver. The reverse transcriptase-polymerase chain reaction was normalised using two housekeeping genes, namely, beta-actin and ubiquitin C. The control value was set at one. Results represent the means \pm standard errors of the means from 3-experiments that were performed in duplicate and compared with sham-irradiated control rats at each time point $(* P<0.05$, based on a one-way analysis of variance). 55 Figure 16: Laser-capture microdissection of rat liver sections after irradiation. A) Liver tissue sections were microdissected in the portal area (a) before cutting and (c) after cutting and in the parenchymal area (b) before cutting and (d) after cutting. B) Polymerase chain reaction (PCR) analysis of the total ribonucleic acid isolated from the microdissected liver tissues from the portal and parenchymal areas. Oligonucleotides specific for the different genes were used. The sizes of the PCR products were analysed using agarose (1\%) gel electrophoresis 
(ultraviolet light picture). Abbreviations: PECAM, platelet/endothelial cell adhesion molecule; NE, neutrophil elastase.

Figure 17: The effect of the intraperitoneal administration of a monoclonal antibody against monocyte chemoattractant protein-1/CCL2 on hepatic gene expression following gamma irradiation. (a) Changes in the levels of messenger ribonucleic acid (mRNA) expression for interferon (IFN)-gamma and for IFN-inducible protein CXCL10 in the rat hepatic tissue following irradiation compared to sham-irradiated control animals using the real-time polymerase chain reaction (RT-PCR). (b) The results obtained using RT-PCR analysis were reassured by running agarose-gel of the polymerase chain reaction products. The RT-PCR data is presented as fold changes. Normalisation of the RT-PCR was achieved using the housekeeping genes, namely, beta-actin and ubiquitin. The control value was set at one. The data represent the mean values from two experiments carried out in duplicate, and the means \pm the standard errors of the means were compared with those from sham-irradiated antibody-treated control animals at each time point $(* P<0.05$, based on a one-way analysis of variance) $\ldots . . . . .58$

Figure 18: Changes in the levels of messenger ribonucleic acid (mRNA) expression measured by real timepolymerase chain reaction for the (a) CXC chemokines and (b) $\mathrm{CC}$ chemokines at different time points in hepatocytes that were administered radiation at a dose of 8 Gy relative to the sham-irradiated control hepatocytes. The data are presented as fold changes. Normalisation of the RT-PCR was achieved using the housekeeping genes, namely, beta-actin and ubiquitin. The control value was set at one. The data represent the means \pm the standard errors of the means of three experiments in duplicate, and they were compared with those of non-irradiated cells served as control at each time point $(* P<0.05$, based on a one-way analysis of variance).

Figure 19: Changes in the levels of messenger ribonucleic acid (mRNA) expression for (a) the CXC chemokines, (b) the CC chemokines and (c) interleukin (IL)-1 beta and IL-6 in liver myofibroblasts (LMFs) at different time points following irradiation at a dose of 8 Gy compared with sham-irradiated control cells. The real time-polymerase chain reaction data that are presented as fold changes in the mRNA expression levels were normalised using two housekeeping genes, namely, beta-actin and ubiquitin $\mathrm{C}$, and the control value was set at one. The data are presented as means \pm standard errors of the mean from three separate experiments carried out in duplicate, and these were compared with those from sham-irradiated cells at each time point $(* P<0.05$, according to a one-way analysis of variance).

Figure 20: The declines in the cycle threshold $(\mathrm{Ct})$ values for CXCL1, CXCL2, CXCL5, CCL2, CCL7, CXCR2, CCR2, IL-1 beta and IL-6 in irradiated and sham-irradiated control rat livers, hepatocytes and liver myofibroblasts. The data were obtained from real-time polymerase chain reaction analysis of the total ribonucleic acid extracted from control and irradiated livers and from isolated liver cells after gamma irradiation. The data are representative of three experiments performed in duplicate, and the means \pm the standard errors of the means are shown for each time point.

Figure 21: The use of radiotherapy in combination with immunotherapy and anti-angiogenic agents (based on a suggestion by Kamrava et al. 2009).

\section{LIST OF TABLES}

Table 1: RT-PCR primers list and sequences

Table 2: Northern Blot sense and anti-sense primer sequences

Table 3: Upregulated ( $\uparrow$ genes in the irradiated rat livers compared with the control livers.

Table 4: Downregulated $(\downarrow)$ genes in the irradiated rat livers compared with the control livers. 


\section{Abbreviations}

$\mathrm{Ab}$

Antibody

ALT

Alanine aminotransferase

AMP Adenosine monophosphate

AP-1

Activating protein 1

APP

Acute phase proteins

APR

Acute phase response

APS

Ammonium persulfate

AST

Aspartate aminotransferase

ATP

Adenosine triphosphate

BCA

Bicinchoninic acid

bp

Base pair

BSA

Bovine serum albumin

cDNA Complementary deoxyribonucleic acid

CPM Counts per minute

$\mathrm{CsCl} \quad$ Cesium chloride

CT Threshold cycle

dd $\mathrm{H}_{2} \mathrm{O} \quad$ Double distilled water

DEPC Diethylpyrocarbonate

DMEM Dulbecco's modified Eagle's medium

DMSO Dimethylsulfoxide

dNTP Deoxyribonucleoside triphosphate

DTT Dithiothreitol

EC Endothelial cells

EDTA Ethylendiaminetetraacetic acid

EGTA Ethylenglycol-bis-(2-aminoethylether)-N, N'-tetraacetate

ELISA Enzyme-linked immunosorbent assay

Fl Fluorescence

FCS Fetal calf serum

FW Fresh weight

g Gravity

GAPDH Glyceraldehyde-3-phosphate dehydrogenase 
Glycoprotein

Gy $\quad$ Gray

$\mathrm{H} \quad$ Hour

HCC Hepatocellular carcinoma

HEPES 2-(4-2-hydroxyethyl)-piperazinyl-1-ethansulfonate

HIF Hypoxia induced factor

HRP Horseradish peroxidase

IFN- $\gamma \quad$ Interferon-gamma

IgG Immunoglobulin $\mathrm{G}$

IL Interleukin

IP Intraperitoneal

$\mathrm{Kb} \quad$ Kilobase

kDa Kilodalton

LCM Laser capture microscopy

LMFs Liver myofibroblasts

LPS Lipopolysaccharide

Min Minutes

MOPS 3-(N-Morpholino)-propanesulfonic acid

NE Neutrophil elastase

NF- $\kappa B \quad$ Nuclear factor $\kappa B$

NO Nitric oxide

OD Optical density

PBS Phosphate buffered saline

PCR Polymerase chain reaction

PECAM Platelet/endothelial cell adhesion molecule

PMSF Phenylmethyl sulfonylfluoride

RB Running Buffer

RILD Radiation-induced liver disease

RMA Robust Multichip Analysis

RNA Ribonucleic acid

RNase Ribonuclease

ROS Reactive oxygen species 
III

rpm Rounds per minute

RT Radiation therapy

r.t. Room temperature

RT-PCR Reverse transcriptase-polymerase chain reaction

SDS Sodium dodecylsulfate

SDS-PAGE SDS-polyacrylamide gel electrophoresis

S.E.M Standard error of the mean

SSC Standard saline citrate

STAT Signal transducer and activator of transcription

TAE Tris acetate EDTA buffer

TEMED N, N, N', N'-tetramethylethylenediamine

TNF- $\alpha \quad$ Tumor necrosis factor alpha

Tris Tris-(hydroxymethyl)-aminomethane

U Unit

UBC Ubiquitin C

UV Ultraviolet

WB Western blot 


\section{SUMMARY}

Radiotherapy is a prospective therapeutic option to treat primary hepatic tumours and liver metastasis, but hepatic radiosensitivity is considered to be a main limitation. To explore the factors underlying for hepatic radiosensitivity, this study investigated the effect of liver focused single-dose gamma-irradiation on healthy rat liver.

The measurement of the serum transaminases levels showed that compared with the control animals, mild hepatocellular damage occurred after irradiation. Specific immunostaining performed on frozen liver sections showed the presence of neutrophils around the portal vessel walls, and these appeared to be in direct contact with the portal smooth muscle actin- and Thy-1-positive liver myofibroblasts (LMFs) 3-6 hours after irradiation. The number of ED1-positive monocytes did not increase following irradiation. Monocyte chemoattractant protein-1 (MCP)-1/CCL2 was observed in the portal vessel walls. Microarray gene expression analysis showed that irradiation upregulated 31 and downregulated 24 genes. The fast and early induction of several leukocyte-attracting CCchemokine and $\mathrm{CXC}$-chemokine genes was observed in the liver tissue at the ribonucleic acid level. Laser capture microdissection pinpointed the gene(s) associated with the migration of the neutrophils to the rat liver after irradiation. A strong and early CCL2 induction was detected in the portal area of the irradiated liver, suggesting an important role for CCL2 within the periportal region after irradiation that induced the recruitment of neutrophil granulocytes into the liver.

Next, the cellular source of the hepatic chemokines, which were involved in neutrophil recruitment, was investigated by irradiating isolated primary liver cell populations. Compared with normal liver tissue, the isolated hepatocytes exhibited a high level of constitutive gene expression for several chemokines, but no major changes in chemokine gene expression were determined after irradiation. Isolated LMFs also showed a constitutive expression of the CCL2, CXCL1, CXCL2, CXCL5 and CXCL8 chemokine genes that was significantly amplified by irradiation. Administering an MCP-1/CCL2 antibody before irradiation significantly increased hepatic interferon-gamma and interferon-inducible protein CXCL10 gene expression, but it did not influence granulocyte recruitment into the portal area.

In contrast to liver tumour patients, healthy liver does not appear to be radiosensitive, which may be because only cirrhotic livers have been irradiated when primary tumours are present. 


\section{INTRODUCTION}

\subsection{Clinical significance of hepatocellular carcinoma}

Hepatocellular carcinoma (HCC) and cholangiocarcinoma comprise the two main types of primary liver cancer, and, of these, HCC is more common, and it is listed as the 6th most frequently diagnosed cancer worldwide. HCC is the $3^{\text {rd }}$ leading cause of malignancyassociated mortalities, and more than half million deaths per year are attributable to this cancer. HCC diagnoses are increasing every day, and every year HCC diagnoses rise in number (Hawkins and Dawson 2006; Parkin 2001). While most of the new cases occur in developing countries, $\mathrm{HCC}$ is also becoming a critical health issue in developed countries, particularly in North America. Furthermore, the incidence of HCC is predicted to increase over the next decade, because of the increasing prevalence of hepatitis $\mathrm{C}$ infection and also because patients with pre-existing liver cirrhosis carry a 1-4\% greater risk of developing HCC (Hawkins and Dawson 2006; Helton et al. 2003). Despite progress in the medical field, the 5year overall survival rate for HCC patients remains stubbornly low at between $3 \%$ and $5 \%$ (Hawkins and Dawson 2006; Parkin 2001). Shortfalls in early diagnoses and the complex nature of the disease are the major problems that affect the treatment of patients with HCC.

\subsection{Treatment of hepatocellular carcinoma}

The standard therapy for HCC comprises liver resection and transplantation (Cheng et al. 2004; Hawkins and Dawson 2006; Iwatsuki et al. 1991), but this is only available to a small number of patients. Given that less than $15 \%$ of the patients with HCC benefit from liver resection and transplantation, efforts are being made to improve the range of treatment options available for patients with HCC (Hawkins and Dawson 2006; Leung et al. 2002; Mathurin et al. 1998). Among the new research areas receiving attention from the National Cancer Institute is the long-term survival of cancer patients. Consequently, the investigators are focusing on the after-effects of therapy and cancer therapies (http://plan.cancer.gov/public/survivor.html; Robbins and Zhao 2007).

Systemic chemotherapy has been considered as an alternative treatment for patients with HCC, but it has been deemed largely ineffective (Hawkins and Dawson 2006; Leung et al. 2002; Mathurin et al. 1998). Another form of therapy for HCC comprises radiation therapy or radiotherapy. 


\subsubsection{Radiotherapy}

Radiotherapy treats cancer effectively, but the subsequent radiotoxicity limits the dose of radiotherapy that can be administered to maximise its efficacy compared with the other therapeutic options that are available. The success of radiotherapy has been limited by its side effects mainly, and applying radiotherapy to organs can affect the healthy tissues that are within the vicinities of the tumours, which can cause radiation-induced functional organ impairment (Emami et al. 1991; Hawkins and Dawson 2006; Leung et al. 2002; Sakata et al. 2007). The consequences of radiotherapy are determined based on its effects on cancerous and healthy tissues in patients, and they include several short- and long-term postradiotherapy effects, deoxyribonucleic acid (DNA) damage (Fig. 1) and chromosomal instability (Qesaraku et al. 2009; Sakata et al. 2007; Morgan and Sowa 2005). Accordingly,

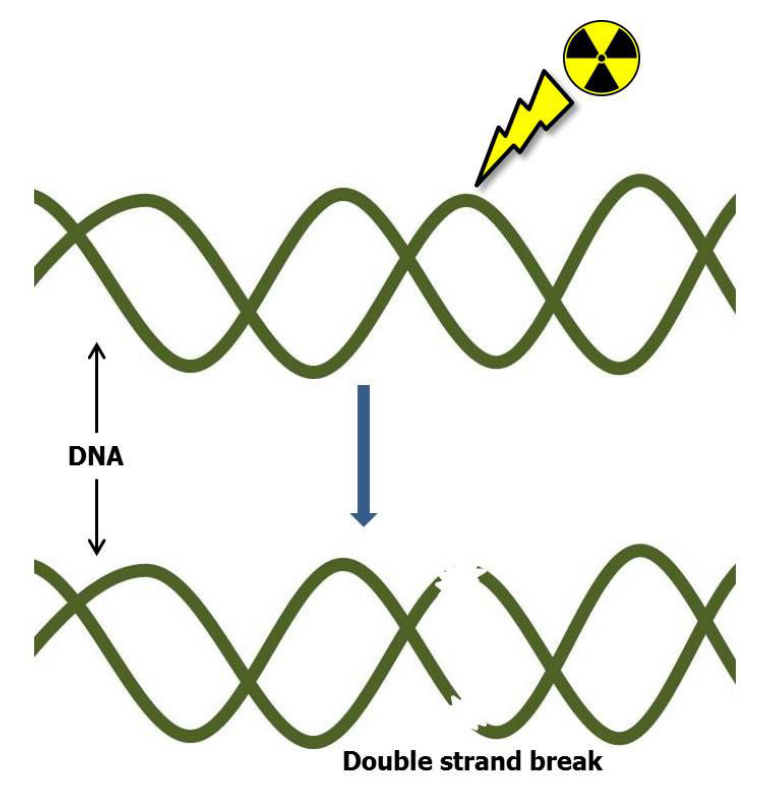

Figure 1: Deoxyribonucleic acid damage caused by ionising radiation (based on suggestion by Morgan and Sowa 2005)

radiation-induced cell death and tissue damage present challenges in the treatment of cancer, which are in addition to the many other consequences of radiotherapy (Hasegawa et al. 2002; Qesaraku et al. 2009). Therefore, if the side effects of radiotherapy can be controlled, it could be administered either alone or in combination with chemotherapy to patients with tumours who may benefit from this form of therapy, and apart from liver (Greco et al. 2004; Moriconi et al. 2008; Wang et al. 1995), its use could be extended to include the treatment of other cancers, for example, malignant lymphomas (Moriconi et al. 2008; Roxin et al. 1978) and biliopancreatic carcinomas (Moriconi et al. 2008; Sempoux et al. 1997). 
Although radiation therapy techniques have been refined and modern radiation techniques, for example, intensity-modulated radiation therapy, have lessened the side effects of radiation on healthy tissues, late radiation effects persist as a central issue in relation to radiotherapy (Robbins and Zhao 2007). This problem can negatively influence the long-term survival of patients who have cancer (Kiltie et al. 1997).

\subsubsection{The liver and radiotherapy}

Radiotherapy has been used for instance in patients with head and neck (Gregoire 2005) and prostate (Christiansen et al. 2007a) cancers, but it has not been applied routinely to patients with cancers of the liver. Many factors contribute to the minor success of radiotherapy in hepatic cancer. For example, the standard dose delivered to the whole liver is 28-35 Gy over a 3-week period, which is very low if tumour elimination is to be achieved, and even this dose harbours a greater than 5\% risk of radiation-induced liver injury (Emami et al. 1991; Hawkins and Dawson 2006; Leung et al. 2002). Therefore, the role of radiotherapy in treating liver cancer has been negligible because of supposed hepatic radiosensitivity (Hawkins and Dawson 2006).

\subsection{Consequences of hepatic radiotherapy}

Radiation-induced injury is the key pathological process underlying radiation-induced liver disease (RILD) (Reed and Cox 1966; Shim et al. 2007). While acute RILD is difficult to diagnose clinically because there are no obvious symptoms, sub-acute RILD is more easily recognised, and the signs become visible from 2 weeks to 4 months after therapy (Christiansen et al. 2006; Lawrence et al. 1995). The retention of hepatic function is crucial to the survival of patients with HCC (Chevret et al. 1999; Hawkins and Dawson 2006). Clinically, the symptoms that are commonly associated with RILD are anicteric hepatomegaly syndrome and ascites. Hence, higher transaminase levels and, especially, serum alkaline phosphatase (AP) levels, are another after effect of radiotherapy (Christiansen et al. 2006; Hawkins and Dawson 2006; Lawrence et al. 1995).

In North America, RILD was the most frequently observed outcome in patients who had undergone external beam radiotherapy (Greco et al. 2004; Hawkins and Dawson 2006; Moriconi et al. 2008; Wang et al. 1995). The consequences of radiotherapy in patients with HCC include the reactivation of viral hepatitis and worsening of a masked liver disease (Cheng et al. 2004; Hawkins and Dawson 2006). 
As yet, the mechanisms underlying the supposed radiosensitivity of the liver leading to RILD have not been explored. Therefore, there is a need to elucidate the poorly understood mechanisms that underlie the liver cell damage that is caused by radiotherapy and the factors that head towards the development of RILD (Cheng et al. 2004; Moriconi et al. 2008; Shim et al. 2007). An understanding of these mechanisms and of the factors involved in RILD development and the subsequent management of radiotoxicity will improve the appeal of radiotherapy as a treatment option for liver cancer (Greco et al. 2004).

\subsection{Radiation-induced inflammation}

The topic of radiation-induced inflammation is not yet well understood and careful investigations on this subject are scarce. An old study has provided some information on a rat ex vivo irradiation model in which ionising radiation caused liver inflammation and injury, after a substantial rise in the number of non-parenchymal liver cells (Geraci and Mariano 1993a). Radiation-induced inflammation is of clinical importance as alcoholic liver disease, viral hepatitis and nonalcoholic steatohepatitis are the most common hepatic disorders that harbour risks of fibrosis and the development of cirrhosis. These disorders are directly associated with persistent inflammation and the harmful substances that are produced as a consequence of oxidative stress, and these have negative influences on the development of these diseases (Parola and Robino 2001). Since radiation-induced tissue damage could likewise activate the inflammatory response, the development of fibrosis might be another hazard for patients who are undergoing radiotherapy (Fig. 2).

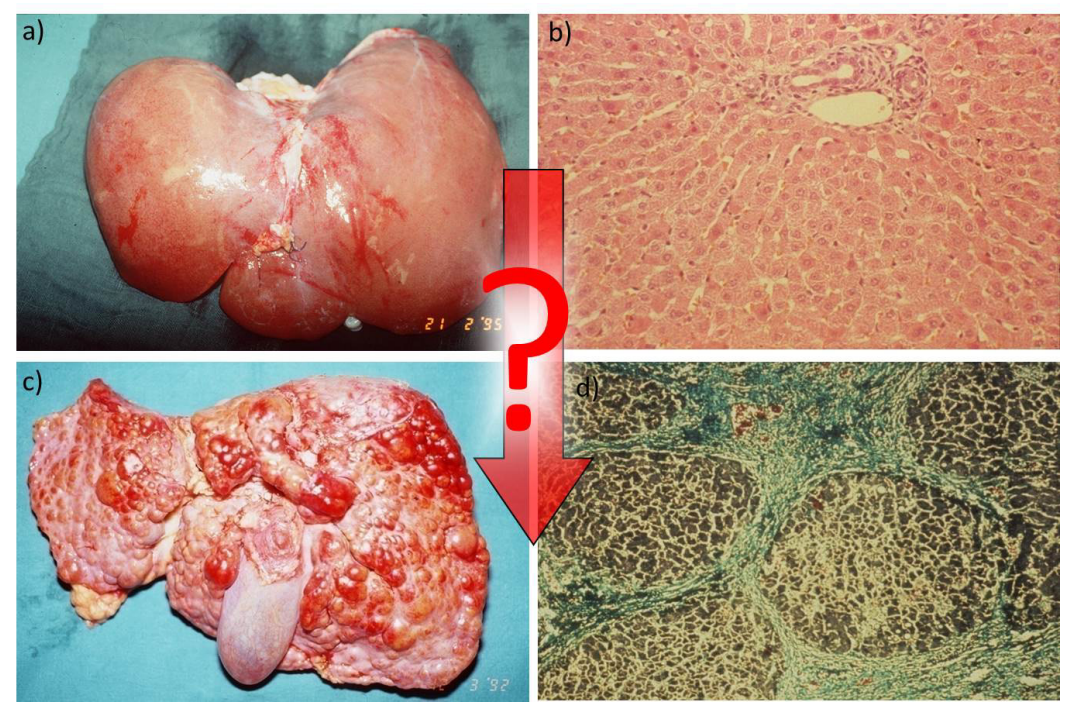

Figure 2: Anatomical and histological images of a normal and a fibrotic liver. (a) A normal liver, (b) a histological section of a normal liver, (c) a fibrotic liver and (d) a histological section of a fibrotic liver. 
Inflammation is the body's biological response to harmful stimuli or physical damage to the tissues or bones, and it is associated with different signs and symptoms. The classic signs of inflammation are heat, redness, pain and swelling (Butterfield et al. 2006; Rocha e Silva 1978). Inflammation reveals a multiple steps development that can be provoked by diverse stimuli that include physical, mechanical or thermal stresses, or the invasion of the body by microorganisms. Inflammation follows a defined order of events irrespective of the causative factor (Butterfield et al. 2006). It includes the flow of particular signalling sequences from specific cells that activate other groups of cells and that activate and recruit white blood cells (WBC) into the stressed or injured area, which is an important step in the inflammatory cascade. Among the WBC, the neutrophils represent the first line of defence (Butterfield et al. 2006; Cannon and St Pierre 1998).

Stressed or injured tissue was considered the only source of the cytokines that induced the mobilisation of neutrophils into the affected area. These neutrophils can generate reactive oxygen species (ROS), which can induce hepatic injury. The role of neutrophils in tissue construction or destruction in the liver has been a topic of discussion (Butterfield et al. 2006; Jaeschke and Hasegawa 2006).

Recent developments in the field of leukocyte biology have emphasised the processes underlying neutrophil recruitment and their roles in normal tissues. Neutrophils are recruited once they pass through the endothelial cell wall of a vessel. Whether this process supports tissue repair or damage during inflammation remains unclear, and answering this question could help to uncover therapeutic approaches (Butterfield et al. 2006; Ramaiah and Jaeschke 2007).

\subsubsection{The roles of cytokines in inflammation}

The mediators of inflammation are diverse and cytokines are the key proteins of this family. The main cytokines are interleukin-6, interleukin-1 beta and tumour necrosis factor (TNF)-alpha. Cells recruited from the circulation are the main sources of several proinflammatory cytokines during hepatic injury or trauma. Whereas interleukin-6 is released mainly from the endothelial cells (EC), macrophages and fibroblasts in an injured area (Ramadori and Christ 1999; Sheikh 2006), mature IL-1 beta can be produced by macrophages in the course or following apoptosis (Perregaux and Gabel 1998; Sheikh 2006). The resident tissues or the mononuclear phagocytes recruited to the damaged area are largely responsible for the production of TNF-alpha. These cytokines are critical for the propagation and 
cessation of the acute phase reaction (APR). The cytokines that influence the APR are diverse. For example, increased levels of the AP-cytokines, namely, IL6, IL-1 beta and TNF-alpha, elevate the APR, whereas other cytokines neutralise the APR proteins (Moshage 1997; Ramadori and Christ 1999; Sheikh 2006).

The major role played by the liver in the host defence mechanism is well understood (Streetz et al. 2001). The hepatocellular stress that is induced by radiation or other toxins may activate the resident macrophages that subsequently release pro-inflammatory mediators, including cytokines (Ramadori et al. 2008), and these cytokines play key roles in modulating the liver's response (Streetz et al. 2001). The induction of these cytokines leads to the consecutive production of further inflammatory mediators and adhesion molecules (Rogler and Andus 1998). The early induction of the pro-inflammatory cytokines and of adhesion molecules following gamma radiation has been reported (Christiansen et al. 2007b; Moriconi et al. 2009). Similarly, prompt increases in the tissue concentrations of these mediators after irradiation may facilitate the transmigration of the inflammatory cells towards the target. Leukocytes may enter the liver tissue through the portal tract where the inflammation has been initiated (Ramadori et al. 2008).

In addition to adhesion molecules and cytokines, the chemokines form another group of inflammatory mediators that mobilise neutrophils in the hepatic vessels (Jaeschke and Hasegawa 2006; Ramadori et al. 2008) and play important roles in the transmigration process (Fig. 3) (Petri et al. 2008).

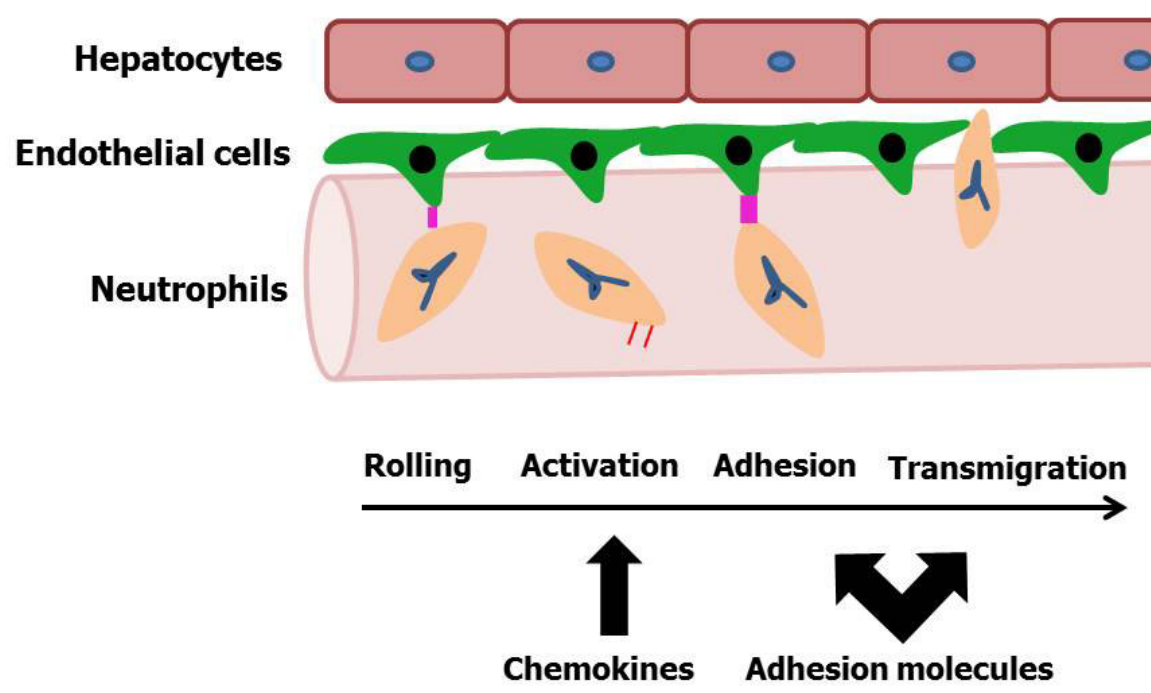

Figure 3: The sequence of the cellular events in the transmigration process (according to Petri et al. 2008, modified and adapted to liver tissue). 


\subsubsection{The roles of chemokines in inflammation}

The chemokines are categorised into several subclasses, of which the $\mathrm{C}, \mathrm{CC}, \mathrm{CXC}$ and CX3C chemokines are the main classes of chemotactic cytokines (Fig. 4). The chemokines

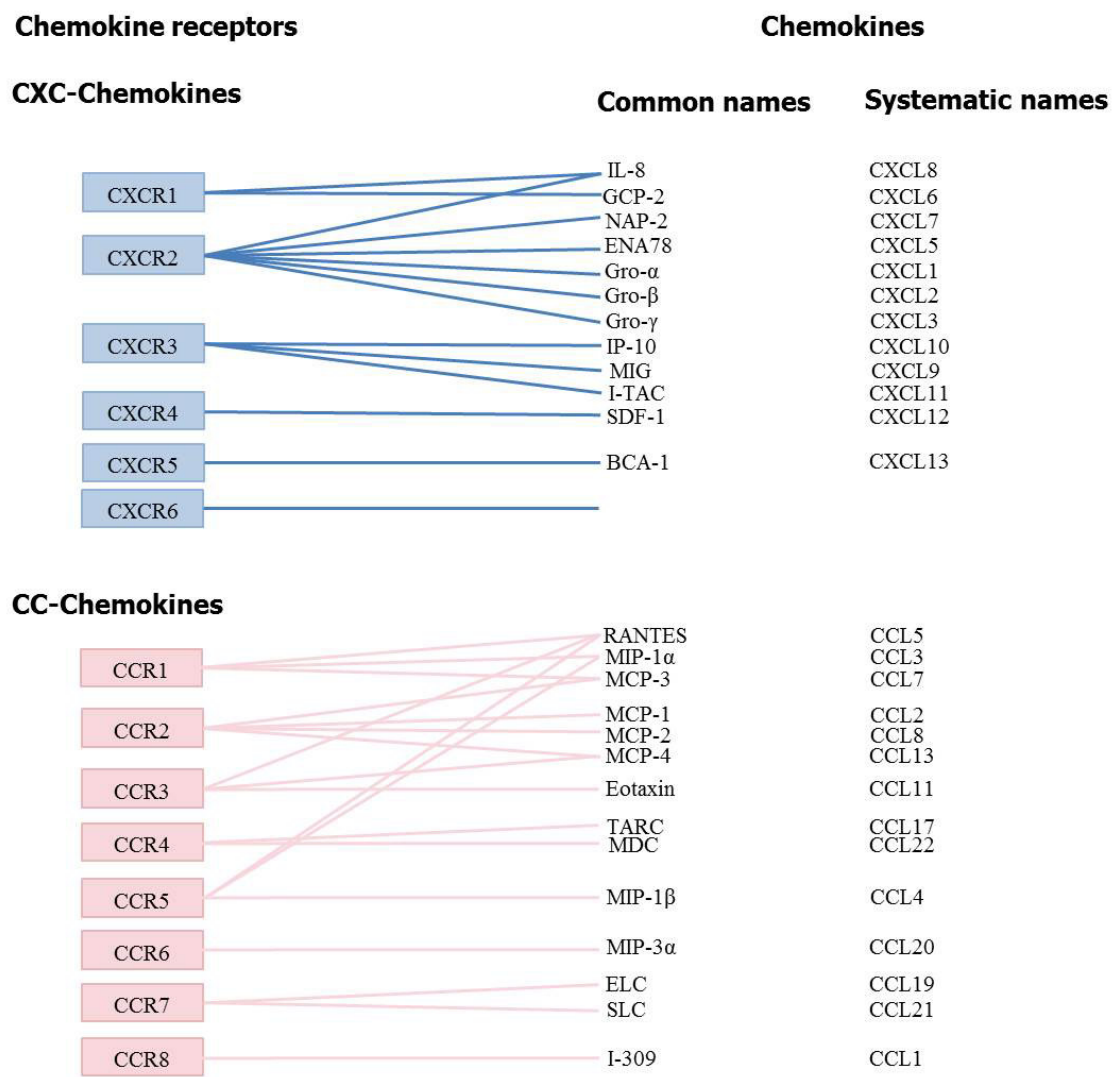

Figure 4: Classification of the main CXC and CC-chemokines and chemokines receptors (modified from Proudfoot 2002).

Abbreviations: IL, interleukin; GCP, granulocyte chemotactic protein; NAP, neutrophil-activating peptide; ENA, epithelial cell-derived neutrophil-activating protein; IP-10, interferon-inducible protein; MIG, monokine induced by gamma interferon; I-TAC, interferon-inducible T-cell alpha chemoattractant; SDF, stromal cell-derived factor; BCA, B cell attracting; RANTES, regulated upon activation normally T-cell expressed and secreted; MIP, macrophage inflammatory protein; MCP, monocyte chemoattractant protein; TARC, thymus and activation regulated chemokine; MDC, macrophage-derived chemokine; ELC, EBI1-ligand chemokine; SLC, secondary lymphoid-tissue chemokine.

are assumed to take part in the process of cell transmigration (Proudfoot 2002). Therefore, it is essential to explore the molecular mechanisms underlying leukocyte transmigration processes and the involvement of the chemokines to gain a deeper understanding of the inflammatory disorders of the liver. Moreover, the chemokines are critical participants in tissue injury and healing, and in the progression of fibrosis (Marra 2002; Le et al. 2004).

The chemokines attract specific populations of leukocytes, and, of these, the CXC chemokines are considered specific for neutrophils. The chemokines are classified according to their structural differences; hence, CXC chemokines can be differentiated from the other 
chemokine classes because of their glycine-leucine-arginine (ELR) motifs (Lin et al. 2007; Proudfoot 2002; Lin 2008). Furthermore, structural differences between human and murine chemokines have been discovered. The human genome contains seven ELR-positive CXC chemokines, but the murine genome contains four: keratinocyte $(\mathrm{KC})$-derived chemokines, namely, CXCL1, which is macrophage inflammatory protein (MIP)-2/CXCL2, which is a lipopolysaccharide (LPS)-induced CXC-chemokine (LIX)/CXCL5 and CXCL15 or lungkine (Bozic et al. 1995; Lin et al. 2007; Proudfoot 2002; Rossi et al. 1999; Wolpe et al. 1989).

IL-8/CXCL8, monokine induced by interferon (IFN)-gamma (MIG)/CXCL9, IFNinducible protein (IP-10)/CXCL10, IFN-inducible T-cell alpha chemoattractant (ITAC)/CXCL11 and stromal cell-derived factor 1 (SDF-1)/CXCL12 belong to the CXC chemokine group. They can mobilise and attract more than one cell population, for example, neutrophils and T- lymphocytes (Harris et al. 1996; Moriconi et al. 2008; Proudfoot 2002). In addition to attracting inflammatory cells such as basophils, monocytes, dendritic cells, $\mathrm{T}$ lymphocytes and natural killer cells to the injured area, the $\mathrm{CC}$ chemokines, for example, monocyte chemoattractant protein (MCP)-1/CCL2, MIP-1 alpha/CCL3, MIP-1 beta/CCL4, MIP-3 alpha/CCL20 and MIP-3 beta/CCL19, also attract a minor proportion of neutrophils (Ajuebor et al. 1998; Moriconi et al. 2008; Proudfoot 2002).

The process of neutrophil recruitment is multifactorial, and it is regulated by a complicated signalling cascade (Frangogiannis et al. 2002) that involves several groups of factors, including CXC chemokines. Among the CXC chemokines involved, IL-8/CXCL8, MIP-2/CXCL2, cytokine-induced neutrophil chemoattractant KC/CXCL1/Gro-alpha, LIX/CXCL5, and CXCR2, which is their main receptor, are specifically involved in neutrophil recruitment (Baggiolini 1998; Chandrasekar et al. 2001). Cell recruitment is governed at many levels, and it could depend on locally produced chemoattractant cytokines, for example, IFN-gamma and TNF-alpha, or on the chemokines that regulate the activities of the cell-surface adhesion receptors and direct the targeted cells to migrate to the affected site (Baggiolini 1998; Gerard and Rollins 2001). These chemokines mainly promote the chemotaxis of the inflammatory cells to the sites of inflammation. Previous studies have shown that MIP-2/CXCL2 and LIX/CXCL5 were induced in myocardial cells in an ischemiareperfusion rat model and after LPS treatment, and that this was followed by neutrophil recruitment (Chandrasekar et al. 2001). Tessier et al. (1997) reported similar results for MIP2/CXCL2. A previous study in a mouse model of ischemia and reperfusion described the need 
for locally expressed KC/CXCL1 and MIP-2/CXCL2 when the liver injury was neutrophil dependent (Lentsch et al. 1998).

Tumour cells express increased levels of chemokines after irradiation (Matsumura et al. 2008). Moreover, radiation alters the tumour microenvironment (TME). Therefore, the modification of these mediators by ionising radiation could be manipulated to improve the benefits of immunotherapy in cancer therapy (Demaria and Formenti 2007; Matsumura et al. 2008). Unfortunately, little information is available that describes radiation-induced inflammation, cell recruitment and the pathways that may be involved in this process. Previous studies have provided some information about the levels of radiation-induced chemokines in cancer cells in which the PI3K/Akt signalling cascade was predicted to be an important pathway; hence, radiation triggers this pathway in tumour cells and in endothelial cells (Matsumura et al. 2008; Zingg et al. 2004), indicating that this signalling cascade is activated in both cell types. Findings from some studies have demonstrated that this pathway is linked to amplification of murine CXCL16 expression and tumour cell survival in an animal model of breast cancer (Ju et al. 2007; Matsumura et al. 2008).

\subsubsection{The role of monocyte chemoattractant protein-1/CCL2 in liver inflammation}

The MCPs are the most studied proteins within the chemokine group of inflammatory mediators. MCP-1/CCL2 belongs to a group of pro-inflammatory chemokines that are released by numerous stressed or damaged tissues (Daly and Rollins 2003). The signalling for MCP-1/CCL2 is conducted through CCR2, which is its main receptor. However, MCP$1 /$ CCL2 can regulate the biological processes that depend on it in the absence of a receptor (Marra et al. 1999; Matsumura et al. 2008; Schecter et al. 2004; Zamara et al. 2007). Several different cell types express MCP-1/CCL2, including monocytes, neutrophils, endothelial cells, epithelial cells, fibroblasts and hepatocytes (Woo et al. 2008; Yoshimura and Takahashi 2007).

In humans, elevated hepatic MCP-1/CCL2 levels were observed during short- and long-term inflammatory disorders. Similar outcomes have been described in several animal models of inflammation (Marra 2002; Zamara et al. 2007). Blocking MCP-1/CCL2 or CCR2 was beneficial in inflammatory conditions and in experimental autoimmune disorders (Huang et al. 2001; Kitagawa et al. 2004). An absence of CCR2 significantly reduced monocyte recruitment to the tissues within mouse models of autoimmune encephalitis (Izikson et al. 2000) and tuberculosis (Peters et al. 2001). Parallel findings were evident in a mouse model of 
atherosclerosis that lacked the CCR2 (Boring et al. 1998). However, there have been conflicting reports about whether MCP-1/CCL2 or CCR2 inhibition induces more tissue injury or infection (Depaolo et al. 2005; Hogaboam et al. 2000). For example, greater hepatic damage was noticed in mice that were deficient in the CCR2 in comparison to wild-type mice. A greater degree of damage was associated with elevated levels of the TNF-alpha and IFNgamma (Hogaboam et al. 2000).

MCP-1/CCL2 and MCP-3/CCL7 are CCR2 agonists, and they have predominant roles in the recruitment of monocytes into injured tissues. The findings from mice models of inflammation showed that the release of monocytes from the bone marrow is strongly impaired in CCR2-deficient mice (Boring et al. 1997; Tsou et al. 2007).

\subsection{Rodent models of radiosensitivity}

Radiotherapy is a leading treatment modality for the elimination of tumours and to prevent their recurrence, but organ radiosensitivity limits the doses of radiotherapy that can be administered. Furthermore, the high doses of radiotherapy used to treat thoracic and abdominal cancers can cause significant injury to non-target organs within these areas. To lessen or prevent the tissue damage that is caused by radiation, it is critical to gather information about the tolerance of organs to radiation, the molecular mechanisms underlying radiation-induced damage and its subsequent repair, and the levels of radiation that reach different tissues. Several rodent models have expedited the research of the impact of radiation on different organs, which have included the effects of partial body irradiation on the lung (elKhatib et al. 1983; Lee et al. 2009; Machtay et al. 2006) and the effects of total body irradiation on the bone marrow (Cui et al. 2002) and lung (Sharplin and Franko 1989) Christiansen et al. (2006) developed a rat model to study the effects of selective irradiation on the liver, which is the model used in the current investigation and the subsequent section describes this model more fully. Using these models, investigators have begun to unravel the mechanisms underlying radiation-induced stress, and information is emerging about the factors that may protect tissues from or inhibit the effects of radiation, which may allow radiation doses to be increased, thereby enabling the elimination of tumour tissues while preserving the integrity of healthy tissues. 


\subsubsection{Rat model of liver radiosensitivity}

As previously mentioned, radiotherapy is associated with the risk of RILD development. Intercellular communication among the different cell systems within the liver could be critical in the development of RILD (Christiansen et al. 2007b). Despite the fact that the liver is sensitive to radiation, hepatocytes are less prone to the effects of radiation, compared with the other cells. Radioresistance of hepatocytes is associated with a varied and oxygen-dependent response to irradiation (Alati et al. 1989a,b; Moriconi et al. 2008). Although healthy hepatocytes are radioresistant, radiation can weaken their defence mechanisms in the presence of inflammatory mediators, which can lead to radiation-induced apoptosis. This programmed cell death in hepatocytes is largely controlled by TNF-alpha (Christiansen et al. 2004; Christiansen et al. 2007b). The threshold level for whole liver irradiation is relatively low, and the likelihood of hepatic damage limits the dose to 20-30 Gy (Anscher et al. 1990; Moriconi et al. 2008). This has been observed in animal studies in which no recovery was evident when the radiation dose exceeded the threshold level, the liver did not return to its normal condition following its exposure to radiation, and hepatic fibrosis and cirrhosis developed (Geraci et al. 1993b; Moriconi et al. 2008). The limitation of the ex vivo rat model used by Geraci et al. (1993b) was surgical extraction of the liver from the abdominal cavity prior to irradiation. Surgical procedures induce an acute phase response which leads to the secretion of cytokines and chemokines (Ueda et al. 2000). Under such conditions, these mediators could have weakened the defense mechanism of the liver cells against radiation and dramatically modified hepatic gene expression. Hence, this model is not suitable for reproducing the clinical situation, because irradiation is administered percutaneously in the clinical setting.

Since the molecular mechanisms underlying RILD are not completely understood (Christiansen et al. 2007b), and a logical research on humans is not feasible, the development of a valid and reproducible model to uncover these mechanisms is essential. Therefore, a rat model of selective percutaneous liver irradiation that does not involve surgical exposure has been established that has facilitated investigations into the consequences of the exposure of the healthy liver to radiation, and this model has demonstrated a release of pro-inflammatory mediators. The induction of these mediators (e.g. cytokines) is central to organism's reaction with respect to inflammatory processes (Butterfield et al. 2006; Christiansen et al. 2006; Christiansen et al. 2007b). To this end, reports from previous studies have indicated that chemokines and cytokines recruit inflammatory cells that may be responsible for cell damage 
and recovery, and eventually for the fibrosis that occurs in the liver following its repeated exposure to toxins (Christiansen et al. 2007b; Jaeschke et al. 1996; Ramadori and Armbrust 2001; Ramadori et al. 2008). Indeed, it is becoming evident that the liver damage provoked by different noxae seems to be induced by inflammatory cells that are found mainly in the different lobules of the liver (Ramadori et al. 2008), whereas the migration of these inflammatory cells is mediated by elevated levels of inflammatory mediators (e.g. chemokines and cytokines) (Jaeschke and Hasegawa 2006). This suggests an active contribution or even a crucial role of these mediators in the tissue damage and repair.

\subsection{Aims of the study}

The liver is affected by the development of primary tumours, including HCC and cholangiocellular carcinoma. The therapeutic options for both types of liver cancer are limited, and there is a need to find new therapeutic prospects. Radiotherapy is emerging as a possible therapeutic option. Unfortunately, the liver is considered to be sensitive to the effects of radiation, and RILD is a severe complication of radiation therapy that is characterised by signs of liver dysfunction and the appearance of ascites (Hawkins and Dawson 2006; Lawrence et al. 1995).

Therefore, it is necessary to study the effects of irradiation on healthy liver to gain insights into the factors responsible for the development of RILD and to protect the healthy tissue from radiation-induced consequences in the vicinities of tumours.

The results from earlier experiments in which rat liver was exposed to a single dose of gamma-irradiation, revealed a mild increase in the levels of the liver enzymes in the serum and changes were observed in the expression of genes in the liver (Christiansen et al. 2006; Christiansen et al. 2007b). Apart from alterations related to iron metabolism (Christiansen et al. 2007b), several genes associated with the pro-inflammatory chemokines were upregulated, such as, CINC-1/CXCL-8, IP-10/CXCL10, ITAC/CXCL11, MCP-1/CCL2, MIG/CXCL9, MIP-1 alpha/CCL3, MIP-1 beta/CCL4, MIP-3 alpha/CCL20, MIP-3 beta/CCL19 and SDF1/CXCL12, but a light microscopic analysis of liver sections from the gamma-irradiated rats did not show significant disruptions to the liver's architecture (Moriconi et al. 2008).

Since it is clear that radiation induces hepatic stress and the release of inflammatory mediators, experiments on primary liver cells, including the hepatocytes and fibroblasts can be expected to provide valuable insights into the mechanisms underlying radiation-induced consequences. These cells can synthesise chemokines and cytokines, moreover, they are 
sensitive to cytokines and exhibit receptors for growth factors, prostaglandins, chemokines, and cytokines. Given the wide range of inflammatory markers that are expressed by liver cells, they can be viewed as interesting targets for counteracting radiation toxicity (Christiansen et al. 2007; Ramadori et al. 2008; Sheikh et al. 2006a).

Based on these previous studies, it was hypothesised that liver cells respond to radiation by releasing certain pro-inflammatory cytokines and chemokines likewise modifying the expression of adhesion molecules. The released mediators, in turn, could attract leukocytes into the liver, thereby inducing inflammatory effects and subsequent tissue injury. This study aimed to

1) identify the localization of the inflammatory cell population(s) within the rat liver lobule following irradiation.

2) further analyse the effect of gamma-irradiation on the expression of chemokine(s) genes that might be involved in recruiting these inflammatory cells into the liver tissue.

3) gain an understanding of the role of MCP-1/CCL2 in the inflammation that is triggered by radiation by blocking its function in vivo.

4) investigate the source of the chemokines participating in the recruitment of inflammatory cells in single liver cell population after irradiation. 


\section{MATERIALS}

\subsection{Animals}

Male rats (Wistar) were bought (Harlan-Winkelmann, Borchen, Germany). The animals (200 g each) were kept in a 12-hour day/night cycle at room temperature and had unlimited access to food ("ssniff®" Spezialitäten $\mathrm{GmbH}$, Soest, Germany) and fresh water. Daily consume of fresh water (12-25 ml) and food pellets (12-15 g) was monitored. All the rats showed a 30-40 g weekly weight gain. After six days of acclimatisation, the animals were involved in the experimental study. The isolation of the hepatocytes was carried out within the first three hours of the day phase. The animals were kept as instructed by institute, NIH rules and the German convention for the animal protection guidelines.

\subsection{Antibodies}

\section{Anti-Neutrophil elastase Ab}

For detection of the neutrophils, immunohistochemical analysis was used with rabbit polyclonal antiserum raised against recombinant human neutrophil elastase (Calbiochem Merck, Darmstadt, Germany). The neutrophil elastase identifies the neutrophils from human and rat.

\section{Anti-CD11b/c Ab}

Neutrophils and macrophages were detected via immunohistochemical analysis, mouse monoclonal antibody raised against rat CD11b/c was used (BD Pharmingen, San Diego CA, USA).

\section{Anti-MCP-1 Ab}

MCP-1 was detected by immunohistological analysis, and goat polyclonal antiserum raised against human MCP-1/CCL2 was used (R\&D Systems, Wiesbaden, Germany). This antibody identified chemokine MCP-1 in rat tissues.

\section{Anti-alpha-SMA Ab}

Fibroblasts were found via immunohistological analysis, and rabbit polyclonal antiserum raised against recombinant human alpha-SMA (Abbiotec, San Diego, USA) was used. This antibody identified fibroblasts in rat tissues. 


\section{Anti-Thy-1 Ab}

Immunohistological analysis was employed to detect the fibroblasts by using mouse monoclonal anti-rat- Thy-1 (Pharmingen, San Diego, CA, USA). This antibody identified fibroblasts in rat tissues.

\section{Anti-rat CXCL1 Ab}

Rat tissue CXCL1 was detectable in immunohistochemical analysis, with rabbit polyclonal antiserum raised against rat CXCL1 (R\&D system, Wiesbaden, Germany). This antibody identified chemokine CXCL1 in rat tissues.

\section{Anti- $\beta$-actin Ab}

$\beta$-actin was recognised using mouse monoclonal antibody (AC-15 clone) raised against N-terminal peptide of $\beta$-actin (Sigma, Munich, Germany). This antibody detected $\beta$ isoform of actin in the tissues of humans, rabbit, bovine, mouse, sheep, rat, pig, dog, guinea pig, carp, chicken, and fruit fly. This antibody showed no cross reaction with the skeletal and cardiac isoforms ( $\alpha$-isoform) of actin.

\section{Anti-rat ED1 Ab}

The detection of rat tissue macrophages was performed via immunohistochemical analysis, in which mouse monoclonal antibody raised against rat spleen cells was used (Serotec, Düsseldorf, Germany). This antibody could identify the 90-100 kDa single chain glycoprotein expressed by most of the rat tissue macrophages.

\section{Anti-rat ED2 Ab}

The Kupffer cells in rat were found through immunohistochemical analysis, using mouse monoclonal antibody raised against the rat spleen cell homogenate (Serotec, Düsseldorf, Germany). This antibody identifies a membrane antigen on Kupffer cells in rat.

\section{Secondary Ab (conjugated horseradish peroxidase):}

Rabbit anti-mouse affinity purified Ig (Dako, Copenhagen, Denmark)

Rabbit anti-goat affinity purified Ig (Dako, Copenhagen, Denmark)

Swine anti-rabbit affinity purified Ig (Dako, Copenhagen, Denmark) 


\section{Secondary Ab (Fluorescent Dye Conjugates):}

Alexa Fluor 350 goat anti-mouse IgG (Invitrogen, Karlsruhe, Germany)

Alexa Fluor 568 donkey anti-rabbit IgG (Invitrogen, Karlsruhe, Germany)

Alexa Fluor 488 donkey anti-mouse IgG (Invitrogen, Karlsruhe, Germany)

\subsection{Proteins, protein standards and enzymes}

Bovine serum albumin (BSA) (PAA Laboratories, Linz, Austria)

Collagenase type I (Biochrom, Berlin, Germany)

Collagenase H (Roche, Mannheim, Germany)

DNase I (Roche, Mannheim, Germany)

Glucose oxidase (Sigma-Aldrich, Munich, Germany)

Insulin (porcine) (Sigma-Aldrich, Munich, Germany)

Pronase E (Merck, Darmstadt, Germany)

Taq DNA polymerase (Invitrogen, Karlsruhe, Germany)

\section{Protease inhibitors}

Antipain (Sigma-Aldrich, Munich, Germany)

Benzamidine (Sigma-Aldrich, Munich, Germany)

Chymostatin (Sigma-Aldrich, Munich, Germany)

Leupeptin (Sigma-Aldrich, Munich, Germany)

Pepstatin A (Sigma-Aldrich, Munich, Germany)

PMSF (Sigma-Aldrich, Munich, Germany)

\section{Stock solutions}

Double-distilled water or RNase-free water was used for stock solutions, which were freshly prepared according to standard lab protocol as described by Sheikh (2006) and Tron 2004), depending on the type of experiment. The $\mathrm{pH}$ of the stock solutions was adjusted at room temperature (r.t.). A $10 \%$ APS solution was prepared and deposited $\left(-20^{\circ} \mathrm{C}\right)$ in $0.1 \mathrm{ml}$ portions. A citric acid solution was prepared with a concentration of $0.25 \mathrm{M}$ and was kept at r.t. A $0.5 \mathrm{M}$ EDTA (disodium salt) solution was prepared, adjusting the $\mathrm{pH}$ at 8 with a $5 \mathrm{~N}$ $\mathrm{NaOH}$ solution using 761-Calimatic pH-Meter (Knick, Berlin, Germany), and was sterilefiltered prior to use. Sodium acetate was brought into solution to achieve a $2 \mathrm{M}$ concentration; the $\mathrm{pH}$ was set to 5.4 using acetic acid and was stored at $4^{\circ} \mathrm{C}$. A $10 \mathrm{x}$ PBS solution was 
prepared consisting of $1.4 \mathrm{M} \mathrm{NaCl}, 100 \mathrm{mM} \mathrm{Na}_{2} \mathrm{HPO}_{4}, 27 \mathrm{mM} \mathrm{KCl}$, and $18 \mathrm{mM} \mathrm{KH}_{2} \mathrm{PO}_{4}$. To adjust the $\mathrm{pH}$ to $7.3, \mathrm{HCl}$ was used. The solution was stored at r.t. after being sterile-filtered. A $20 \%$ solution of SDS was achieved by stirring and warming until SDS was dissolved using magnetic-mixer model type M21/1 (Framo-Gerätetechnik, Eisenbach, Germany) until the liquid became clear. Sodium citrate was used to prepare a $0.25 \mathrm{M}$ solution which was kept prior to use. The $\mathrm{pH}$ was adjusted to 7.0 with a $0.25 \mathrm{M}$ citric acid solution. A $2 \mathrm{M}$ Tris- $\mathrm{HCl}$ solution was prepared with a $\mathrm{pH}$ of 7.4 , stored $\left(4^{\circ} \mathrm{C}\right)$ after sterile-filtering prior to use.

\subsection{Chemicals}

All analytical grade chemicals employed were purchased from companies as listed below:

Alpha ${ }^{32}$ phosphate labeled deoxycytidine-triphosphate and Ficoll ${ }^{\circledR} 400$ were purchased from Amersham Pharmacia Biotech (Freiberg, Germany), dNTP master mix from Bioline (Luckenwalde, Germany), fetal calf serum (FCS), medium 199 and trypan blue from Biochrom (Berlin, Germany). Ampuwa ${ }^{\circledR}$ water was bought from Fresenius (Bad Homburg, Germany). The $A G \AA 501$ mixed-bed resins and guanidine-isothiocyanate for RNA-isolation were obtained from Bio-Rad (Munich, Germany) and Invitrogen (Karlsruhe, Germany), respectively. GeneRuler ${ }^{\mathrm{TM}} 100 \mathrm{bp}$ DNA ladder plus and 6x loading dye solution were purchased from MBI Fermentas (Vilnius, Lithuania).

All the usual laboratory chemicals bought from Merck (Darmstadt, Germany) are as follow: acetone, acetic acid, beta-mercaptoethanol, bromophenol blue, ethyl alcohol, formaldehyde (37\%), formamide, glucose, glycerol, glycerol-gelatin, hematoxylin solution Mayer's, methanol, penicillin G and TEMED.

L-glutamine from PAA (Linz, Austria), cesium chloride from Paesel and Lorei (Frankfurt, Germany), and glycine sodium dodecyl sulfate (SDS) were obtained from Roth (Karlsruhe, Germany). A Tris-HCl was purchased from Serva (Heidelberg, Germany). Antifoam A, ammonium persulfate, citric acid, dexamethasone, 3,3-diaminobenzidine, DTT, DMSO, ethidium bromide, EDTA, MOPS, HEPES, sodium acetate, sodium azide, Nlauroylsarcosine, Triton ${ }^{\mathrm{TM}} \mathrm{X}-100$, sodium citrate were purchased from Sigma-Aldrich Chemie (Munich, Germany). QuikHyb® hybridisation solution for Northern blot was purchased from Stratagene (Heidelberg, Germany) 


\subsection{Additional materials}

For the animals experiments, Braunules $2 \mathrm{G} 14$ were purchased from Braun (Melsungen, Germany). Nick column, Sephadex G-50 M DNA grade and Hybond N nylon membrane were bought from GE Healthcare, (Freiburg im Breisgau, Germany). Culture dishes $(0.6 \mathrm{~cm})$ and falcon tubes of $15 \mathrm{ml}$ and $50 \mathrm{ml}$ and syringes of different size of 2, 5 and $20 \mathrm{ml}$ were obtained from Becton, (Dickinson, NJ, USA). Hybridisation glass tubes from Biometra (Munich, Germany) and for the centrifugation, polyallomer-tubes (Thinwall, $5 \mathrm{ml}$ ) from Beckman (Munich, Germany), which were commercially available and purchased. Safelock tubes in different sizes (0.2- $2 \mathrm{ml}$ ) from Eppendorf (Hamburg, Germany) and disposable pipettes for cell culturing ranging from 2 to $50 \mathrm{ml}$ were obtained Sarstedt (Nümbrecht, Germany). The scintillation tubes of $5 \mathrm{ml}$ size were bought from Zinsser Analytic (Frankfurt, Germany). Sterile filter tips from Biozym (Oldendorf, Germany) and sterile filter Nalgene of $0.2 \mu \mathrm{m}$ size were purchased from Sartorius (Göttingen, Germany). The X-ray films were bought from Amersham Biosciences, (Freiburg, Germany) to develop Northern and Western blot membranes.

\subsection{Technical equipment}

The technical instruments that were utilised in this thesis are mentioned on appropriate position where they have been used.

\section{METHODS}

\subsection{Cellular biology techniques}

\subsubsection{Whole liver irradiation in vivo}

The rats were irradiated based on the established lab protocol described earlier by Christiansen et al. (2006, 2007).

In short, the SOMATOM ${ }^{\circledR}$ Spirit Balance scanner (Siemens Medical Solutions, Erlangen, Germany) was used for planned computed tomography (CT), in which the livers of the rats were defined prior to irradiation. The rats were subsequently subjected to anesthesia intraperitoneally (IP) with ketamine (at $90 \mathrm{mg} / \mathrm{kg}$ per dose) (Intervet, Unterschleissheim, Germany) and 2\% xylazine (7.5 mg/ kg per dose) (Serumwerk Bernburg, Bernburg Saale, Germany) based on body weight. 
The distribution of dose was calculated after marking the boundaries on the skin of rat. A $6 \mathrm{MeV}$ photons dose was administered to the livers selectively at a rate of $2.4 \mathrm{~Gy} / \mathrm{min}$ utilising Clinac $600 \mathrm{C}$ accelerator (Varian, Palo Alto, Calif).

Following the anteroposterior and posteroanterior treatment technique, the liver was irradiated with $25 \mathrm{~Gy}$, in a single dose. For the specific control sham-irradiated animals were used. The controls and treated animals were anesthetised and transported at the same time. Under careful supervision the rats were sacrificed humanely, at specific time points, post irradiation $(1,3,6,12,24,48 \mathrm{~h}$ ). For each point in time (number of animals $=5$ ), the serum and livers were taken and frozen.

Another experiment was performed where the single dose $(250 \mu \mathrm{g} / \mathrm{kg})$ of monoclonal anti-rat MCP-1/CCL2 antibody was injected intraperitoneally (IP) to the rats before 30 minutes to irradiation. The antibody was dissolved in $0.5 \mathrm{ml}$ sterile sodium chloride $(0.9 \%)$. The animals were divided into 4 groups $(\mathrm{G})$. G1: only received irradiation (25 Gy); G2: received MCP-1/CCL2 antibody; G3: received both antibody MCP/CCL2 and irradiation; G4: received only saline injection and sham-irradiated, served as controls. The animals of all four groups ( $\mathrm{n}=2$ for each group) were sacrificed after 3 and 6 hours of irradiation. All animals were anesthetised and irradiated according to the protocol described above.

\subsubsection{Isolation of rat hepatocytes}

The male Wistar rats were used to isolate the hepatocytes. The hepatocytes were isolated by circulating the perfusion with collagenase basically implementing the method described by Katz et al. (1979) and Seglen (1972) but incorporating some modifications in keeping our standard laboratory protocol as described by Sheikh (2006) and Tron (2004) including preparation of solutions and medium.

\subsubsection{Liver perfusion}

The vena portae were cannulated post laparotomy. Then ligation of the inferior vena cava was done to block the perfusion media from flowing into the whole body circulation. Next, a cut was made in the inferior vena cava just below the liver and cannulated. Using the non-recirculating mode through the portal vein of the liver, it was perfused using Peristaltic pump P-1 (Amersham, Freiburg, Germany) with pre-perfusing medium supplemented by $\mathrm{CO}_{2}$ with a $30 \mathrm{ml} / \mathrm{min}$ flow rate until the liver showed no traces of blood. Next, the break-down of the extracellular matrix components was done by perfusing the liver in the recirculating mode 
using the collagenase perfusion medium. This was continued for about 7-11 minutes till the liver began to feel soft.

\subsubsection{Hepatocytes suspension}

After the liver was perfused, the excised hepatic tissue was placed in a glass beaker which was sterilized. The beaker contained the medium 199 with supplements. The collagen tissue surrounding the liver, Glisson's capsule, was removed and discarded. The cell suspension was prepared by disrupting the tissue mechanically utilising sterile-forceps and the suspension was filtered through a $79 \mu \mathrm{m}$ nylon mesh, in order to remove remaining cell aggregates, connective tissue and hepatic capsule. After addition of wash medium, the filtrate was centrifuged at $20 \times \mathrm{g}\left(4^{\circ} \mathrm{C}, 2 \mathrm{~min}\right)$ using a HettichRotina 3850 (Hettich, Tuttlingen, Germany) to discard non-parenchymal cells and fragments.

Once the final centrifugation was completed, the pre-warmed $\left(37^{\circ} \mathrm{C}\right.$; water bath 1083 , GFL, Burgwedel, Germany) supplemented medium 199 was used to resuspend the hepatocytes. After a further centrifugation (conditions as above), $50 \mathrm{ml}$ of medium/g pellet (FW) were added to attain a cells density of $10^{6} / 2.5 \mathrm{ml}$ (as previously used by Sheikh 2006 and Tron 2004).

\subsubsection{Media and solutions for hepatocytes preparation and culture}

Double-distilled water was used to prepare all the media and solutions for cell culture. It was stored at $4{ }^{\circ} \mathrm{C}$ after undergoing further purification via sterile filtration. Before isolation, the preparation of all solutions was carried out within the preceding $24 \mathrm{~h}$.

A Krebs-Ringer stock solution $\left(120 \mathrm{mM} \mathrm{NaCl}, 1.2 \mathrm{mM} \mathrm{MgSO}{ }_{4} \times 7 \mathrm{H}_{2} \mathrm{O}, 4.8 \mathrm{mM} \mathrm{KCl}\right.$, $24.4 \mathrm{mM} \mathrm{NaHCO} 3,1.2 \mathrm{mM} \mathrm{KH}_{2} \mathrm{PO}_{4}$ ) was equilibrated to $\mathrm{pH} 7.35$ using carbogen. A medium for pre-perfusion was prepared in 1x Krebs-Ringer solution containing $0.25 \mathrm{mM}$ EGTA. A collagenase perfusion medium in $1 \mathrm{x}$ Krebs-Ringer solution $\left(4 \mathrm{mM} \mathrm{CaCl} \cdot 2 \mathrm{H}_{2} \mathrm{O}, 15 \mathrm{mM}\right.$ HEPES, $50 \mathrm{mg}$ collagenase) was prepared immediately before isolation. It was equilibrated by using carbogen $30 \mathrm{~min}$ before sterile filtration. A wash medium $(120 \mathrm{mM} \mathrm{NaCl}, 20 \mathrm{mM}$ HEPES/NaOH pH 7.4, $4.8 \mathrm{mM} \mathrm{KCl,} 1.2 \mathrm{mM} \mathrm{KH}_{2} \mathrm{PO}_{4}, 1.2 \mathrm{mM} \mathrm{MgSO}{ }_{4} \cdot 7 \mathrm{H}_{2} \mathrm{O}, 0.4 \%$ Bovine serum albumin) was prepared. Furthermore, a medium 199 containing Earle's salts devoid of $\mathrm{NaHCO}_{3}$ (5.5 mM Glucose $\cdot \mathrm{H}_{2} \mathrm{O}, 18 \mathrm{mM} \mathrm{NaHCO}$, $15 \mathrm{mM}$ HEPES, 0.4\% Bovine serum albumin) was prepared. Immediately before isolation, carbogen was used for equilibrating the medium to $\mathrm{pH} 7.35$, followed by sterile filtration. 


\subsubsection{Isolation of rat liver myofibroblasts}

The main hepatic non-parenchymal cells are myofibroblasts, EC and Kupffer cells. Isolation of liver non-parenchymal cells was performed by removing the hepatocytes after perfusion with collagenase. This step was done by low-speed centrifugation. Liver nonparenchymal cells were isolated by Nycodenz gradient and separated into Kupffer cells and myofibroblasts fractions thereafter using centrifugal elutriation. For this purpose, J21 centrifuge equipped with JE-6 elutriator rotor (Beckmann Instruments, Palo Alto, Calif., USA) was used. The detailed method of myofibroblasts isolation including the solutions used was adapted from the methods part of Kupffer cells isolation from Tron (2004). This protocol was modified in our lab from method described by Knook and Sleyster (1976). After isolation of the myofibroblasts, the culturing of cells was performed to suit our lab protocol as described by Dudas et al. (2007) incorporating some changes.

\subsubsection{Liver perfusion and preparation of cell suspension}

The laparotomy and cannulation were performed in line with the protocol described above (see 3.1.2). Perfusion of the liver was performed using the pre-perfusion medium containing Gey's Balanced Salt Solution (GBSS) and sodium hydrocarbonate. Enzyme solution 1 containing pronase with subsequent changes to enzyme solution 2 which was composed of pronase and collagenase was used for further perfusion.

After subsequent excision of hepatic tissue, this was transferred to an enzyme solution 3 composed of pronase, collagenase and DNase I, in sterile Petri dish. Next, it was mechanically disrupted using sterile forceps. The cell suspension was then stirred for 30 minutes in the same perfusion solution, simultaneously maintaining the $\mathrm{pH}$ at 7.5. After filtering it through a sterile sieve, the cell suspension was collected in $50 \mathrm{ml}$ polypropylene tubes. The main components including the hepatocytes and other large cell aggregates of the liver were removed by centrifuging the cell suspension $\left(35 \mathrm{~g}, 4^{\circ} \mathrm{C}, 4 \mathrm{~min}\right)$. Thereafter, the centrifugation $\left(640 \mathrm{~g}, 5 \mathrm{~min}, 4^{\circ} \mathrm{C}\right)$ of supernatant was done one more time. The resuspension of the pellet was carried out in $50 \mathrm{ml}$ of GBSS containing $100 \mu \mathrm{l}$ DNase I.

\subsubsection{1a. Separation of the non-parenchymal liver cells}

Employing the procedure according to Tron (2004) the non-parenchymal liver cells were separated using $\mathrm{Nycodenz}^{\circledR}$ density gradient. The cell suspension was distributed to four sterile $50 \mathrm{ml}$ polypropylene tubes and centrifuged $\left(640 \mathrm{~g}, 5 \mathrm{~min}, 4^{\circ} \mathrm{C}\right)$. After disposing the 
supernatant, the resuspension of the pellet was carried out in 5-6 ml of GBSS with $100 \mu 1$ DNase I and combined together in one sterile $50 \mathrm{ml}$ polypropylene tube. The volume was then adjusted to $24 \mathrm{ml}$ by adding $14 \mathrm{ml}$ of $30 \%$ Nycodenz. This mixture was transferred into four sterile $15 \mathrm{ml}$ polypropylene tubes. Then the contents of the tubes were carefully layered over using GBSS (1.5 $\mathrm{ml}$ per tube) and the centrifugation $\left(1,800 \mathrm{~g}, 15 \mathrm{~min}, 4^{\circ} \mathrm{C}\right)$ of gradient was carried out. Next, the brown interphase layer seen between the Nycodenz and the GBSS containing the non-parenchymal liver cells was carefully transferred into a polypropylene tube $(50 \mathrm{ml})$. Afterwards, it was centrifuged $\left(640 \mathrm{~g}, 4^{\circ} \mathrm{C}, 5 \mathrm{~min}\right)$.

\subsubsection{1b. Purification of myofibroblast by counterflow elutriation}

The pure culture of the myofibroblasts was drawn by centrifugal counterflow from the non-parenchymal liver cells (myofibroblasts, Kupffer cells and endothelial cells) following the method of Knook and Sleyster (1976) incorporating some modifications to suit our laboratory protocol as given by Tron (2004). As stated above, a similar protocol was employed for the Kupffer cells and myofibroblasts. This procedure too was adapted from Tron (2004).

The pellet thus obtained was resuspended in 5-6 $\mathrm{ml}$ of $0.4 \% \mathrm{BSA} / \mathrm{GBSS}$. Next, the collection was injected using a sterile $10 \mathrm{ml}$ syringe in the elutriation system. Following the manufacturer's instructions the JE-6B elutriation rotor was assembled. It was then spun at 2,500 rpm in a J2-21 centrifuge (Beckman, Munich, Germany). The fractions enriched with sinusoidal endothelial cells, myofibroblasts and Kupffer cells were gathered at $19 \mathrm{ml} / \mathrm{min}, 23$ $\mathrm{ml} / \mathrm{min}$ and $55 \mathrm{ml} / \mathrm{min}$ of the flow rates, respectively. The myofibroblasts fraction of the sedimentation was achieved by centrifugation $\left(640 \mathrm{~g}, 4^{\circ} \mathrm{C}, 5 \mathrm{~min}\right)$. Using a Neubauer chamber, the number of living cells was observed by Trypan-blue staining.

\subsubsection{1c. Media and solutions for myofibroblasts}

The preparation of entire range of media and solutions was carried out in $\mathrm{ddH}_{2} \mathrm{O}$. Thereafter, they were sterile-filtered and kept at $4^{\circ} \mathrm{C}$, unless mentioned otherwise. The solutions and method of myofibroblasts isolation were followed from the section of Kupffer cells preparation and culture by Tron (2004). A 10x Gey's Balanced Salt Solution (GBSS) (10 g Glucose, 3.7g KCl, 0.7g $\mathrm{MgSO}_{4} \times 7 \mathrm{H}_{2} \mathrm{O}, 80 \mathrm{~g} \mathrm{NaCl}, 1.7 \mathrm{~g} \mathrm{NaH}_{2} \mathrm{PO}_{4} \times \mathrm{H}_{2} \mathrm{O}, 2.2 \mathrm{~g} \mathrm{CaCl}_{2} \times 2 \mathrm{H}_{2} \mathrm{O}$,

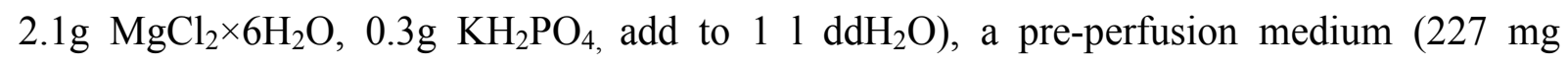
$\mathrm{NaHCO}_{3}, 100 \mathrm{mll0x}$ GBSS, add to $11 \mathrm{ddH}_{2} \mathrm{O}$ ) that was prepared immediately before isolation; adjustment of the $\mathrm{pH}$ was performed to 7.4., and a 1x GBSS without $\mathrm{NaCl}$ (370 mg 
$\mathrm{KCl}, 170 \mathrm{mg} \mathrm{NaH}{ }_{2} \mathrm{PO}_{4} \times \mathrm{H}_{2} \mathrm{O}, 70 \mathrm{mg} \mathrm{MgSO}_{4} \times 7 \mathrm{H}_{2} \mathrm{O}, 220 \mathrm{mg} \mathrm{CaCl} \times 2 \mathrm{H}_{2} \mathrm{O}, 30 \mathrm{mg} \mathrm{KH}_{2} \mathrm{PO}_{4}$, $227 \mathrm{mg} \mathrm{NaHCO}, 210 \mathrm{mg} \mathrm{MgCl}_{2} \times 6 \mathrm{H}_{2} \mathrm{O}, 1 \mathrm{~g}$ Glucose, add to $11 \mathrm{ddH}_{2} \mathrm{O}$ of which the $\mathrm{pH}$ was adjusted to 7.4, were prepared. Furthermore, a 30\% Nycodenz ${ }^{\circledR}\left(30\right.$ g Nycodenz ${ }^{\circledR}$, add to 100 $\mathrm{ml} 1 \mathrm{x}$ GBSS without $\mathrm{NaCl}$ ) was produced, the solution was distributed in $14 \mathrm{ml}$ aliquots and stored at $-20^{\circ} \mathrm{C}$. A $0.4 \% \mathrm{BSA} / \mathrm{GBSS}$ was achieved by dissolving $2 \mathrm{~g}$ BSA in $500 \mathrm{ml} 1 \mathrm{x}$ GBSS.

Enzyme solutions: All the enzymes were dissolved in GBSS (30 min, r.t.), with occasional agitation. The enzyme solution 1 was prepared by mixing $120 \mathrm{mg}$ Pronase E in 60 $\mathrm{ml}$ 1x GBSS, enzyme solution 2 was prepared by adding $75 \mathrm{mg}$ Pronase E and $80 \mathrm{mg}$ collagenase $\mathrm{H}$ into $150 \mathrm{ml}$ 1x GBSS. The enzyme solution 3 was produced by dissolving 20 mg Pronase E, and $60 \mathrm{mg}$ collagenase $\mathrm{H}$ in $100 \mathrm{ml}$ 1x GBSS. $300 \mu \mathrm{l}$ of DNase I stock (100

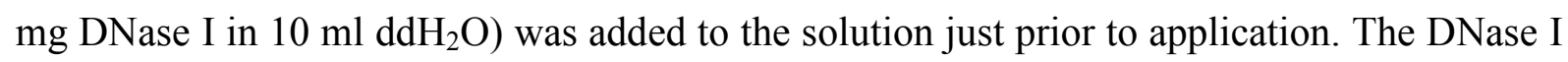
stock was stored at $-20^{\circ} \mathrm{C}$ in $1000 \mu 1$ portions.

\subsubsection{Primary culture of hepatic cells: treatment and harvesting}

Hepatocytes and myofibroblasts were cultured in a sterile environment (TL-2472, Laminar-Air flow cabinet, Heraeus, Hanau, Germany) in a carbogen athmosphere, at $100 \%$ humidity and $37^{\circ} \mathrm{C}$ as described by Sheikh (2006) and Tron (2004) in the incubator (HeraeusElectronic, Hannover, Germany)

\subsubsection{Rat hepatocytes culture}

For promoting cell adhesion on polysterol dishes, FCS was added $(4 \mathrm{ml} / 100 \mathrm{ml}$ cell suspension). Thereafter, antibiotics (1 $\mathrm{ml}$ penicillin/streptomycin stock solution) and permissive hormones $\left(10^{-7} \mathrm{M}\right.$ dexamethasone, $10^{-9} \mathrm{M}$ insulin) were added, to achieve a volume of $100 \mathrm{ml}$. Plating of hepatocytes was carried out on plastic dishes $\left(60 \mathrm{~mm} ; 2 \times 10^{6}\right.$ cells/dish). Subsequently, the early adhesion stage, which extended for 4 hours, the hepatocytes were incubated after changing the medium 199 at conditions described above, but in the absence of FCS. Each $60 \mathrm{~mm}$ culture dish received a volume of $2.5 \mathrm{ml}$ medium.

Twenty-four hours after the hepatocytes were plated, irradiation was done. Care was taken to replace the culture medium immediately prior to the irradiation: On day one following the isolation, the hepatocytes underwent irradiation ( $8 \mathrm{~Gy}$ ) by following the conditions described above (see 3.1.1). The experimental control (sham-irradiated) dishes contained the same quantity of media and cells. The sham-irradiated controls and irradiated cells were harvested simultaneously at the time points mentioned (i.e. 1, 3, 6, 12, 24 h). Next, 
PBS ( $\mathrm{pH}$ 7.4) was used for washing the cells which were thereafter stored at $-80^{\circ} \mathrm{C}$ for further RNA extraction.

\subsubsection{Antibiotic and hormone stock solutions}

The solution was stored at $-20^{\circ} \mathrm{C}$ in portions after sterile filtration. The recipe of the solutions is similar to that given in Tron (2004): The pen/strep stock solution was prepared by dissolving $0.64 \mathrm{~g}$ penicillin $\mathrm{G}$ (sodium salt), and $1.17 \mathrm{~g}$ streptomycin sulfate into $100 \mathrm{ml} 0.9 \%$ $\mathrm{NaCl}$. Dexamethasone (3.92 g) was dissolved in $0.3 \mathrm{ml}$ of ethanol. Using $0.9 \% \mathrm{NaCl}$ the solution was brought to $100 \mathrm{ml}$. Insulin (6 mg) was dissolved in $100 \mathrm{ml} 0.9 \% \mathrm{NaCl}$ set to $\mathrm{pH}$ 2.5 and neutralized, followed by addition of BSA (100 mg).

\subsubsection{Culturing and irradiation of liver myofibroblasts (LMF)}

In accordance with our laboratory established protocol as given by Dudas et al. (2007) the rat liver myofibroblasts were cultured in DMEM (Biochrom, Berlin, Germany) containing FCS (15\%), penicillin $(100 \mathrm{U} / \mathrm{ml})$, streptomycin $(100 \mu \mathrm{g} / \mathrm{ml})$ and L-glutamine (1\%). Two days after plating, the culture medium was replaced and thereafter every second day. Keeping the temperature at $37^{\circ} \mathrm{C}$ with $5 \% \mathrm{CO}_{2}$ atmosphere and $100 \%$ humidity, the cells were kept in culture. When they reached confluence, passage was performed. The cells were then used four days post the first passage. Utilising $8 \mathrm{~Gy}$, as mentioned above (see 3.1.1), LMF thus isolated were irradiated as the irradiation protocol described by Christiansen et al. (2007). The dishes with the experimental controls (sham-irradiated) contained the same quantity of media and cells. The sham-irradiated controls and irradiated cells were simultaneously harvested as mentioned at 1, 3, 6, 12, 24 hours points in time. The cells were later washed by PBS ( $\mathrm{pH} 7.4$ ), stored for RNA extraction at $-80^{\circ} \mathrm{C}$.

\subsubsection{Immunohistology and immunofluorescence staining}

Immunohistology and immunofluorescence staining was carried out as described before (Dudas et al. 2007) incorporating slight changes. Immunohistology was performed according to peroxidase staining method. Utilising Frigcout 2800E Cryostat (Leica Bensheim, Germany), cryosections $(5 \mu \mathrm{m})$ of the liver were cut and fixed $\left(10 \mathrm{~min},-20^{\circ} \mathrm{C}\right.$ cold acetone). Afterwards the sections were incubated for 60 minutes with endogenous peroxidase solution consisting of glucose/ glucose oxidase/ sodium azide for blocking. This step was followed by incubation of sections with FCS for 30 minutes to control the non-specific staining. Then slides were rinsed using cold PBS for 3 times followed by incubation with primary antibodies 
directed against NE, ED1, ED2, CD11b/c, and MCP1/CCL2 diluted 1: 100 in PBS (1 h, r.t.), respectively. Later on, the slides were rinsed thrice in cold PBS and incubated with secondary antibodies for 60 minutes with peroxidase-conjugated anti-rabbit/ anti-mouse/ anti-goat immunoglobulins pre-absorbed with normal rat serum. Cold PBS was used to rinse the sections and incubated with solution containing 3,3-diaminobenzidine $(0.5 \mathrm{mg} / \mathrm{ml})$ and $\mathrm{H}_{2} \mathrm{O}_{2}$ (0.01\%), dissolved in PBS (10 $\mathrm{min})$ to observe the immune complexes. Thereafter, nuclei was counterstained by Hematoxylin solution Mayer's. Finally the slides were covered with coverslips using pre-warmed $\left(65^{\circ} \mathrm{C}\right)$ glycerin-gelatin.

For double-immunofluorescence staining, sections were rinsed with PBS and thereafter incubated $(60 \mathrm{~min}$.) in blocking solution at r.t. as mentioned above. The primary antibodies dilutions were applied for 60 minutes at r.t. Different combinations were applied in double-immunofluorescence staining: mouse monoclonal antibody directed against rat CD11b/c together with ED1, anti-CD11b/c with anti-SMA, anti-CD11b/c with anti-rat Thy-1. All primary antibodies were dissolved in PBS before incubation. The sections were rinsed thrice (each 5 minutes) using cold PBS and incubated for 60 minutes.

Corresponding Alexa-fluor-conjugated secondary antibodies Alexa- 350 \& 488 (1: 200) and Alexa-568 (1: 400) were used to detect primary antibodies.

Non-immune serum was taken as negative control. Afterwards slides were rinsed again with PBS thrice (5 minutes each) and sections were incubated with 4',6-diamidino-2phenylindole (DAPI) (Invitrogen, Darmstadt, Germany) solution. The sections were washed with PBS and with $\mathrm{ddH}_{2} \mathrm{O}$. Subsequently the sections were covered with Fluoromount-G $\mathrm{G}^{\mathrm{TM}}$ (SouthernBiotech, Birmingham, USA) and were analysed by fluorescent microscopy MC 100 Spot (Carl Zeiss, Jena, Germany).

\subsubsection{Laser capture microdissection (LCM)}

After irradiation the rat liver sections were dissected from the frozen tissues (three for each time point and three regions per section). The portal region and liver parenchyma were micro-dissected by LCM (Carl Zeiss, Jena, Germany) following the protocol described earlier Espina et al. (2006) with slight modifications. The liver cryostat sections were cut into $10 \mu \mathrm{m}-$ thick slices and placed on RNAse free slides. The liver sections were fixed in varying concentrations of ethanol and RNAse hematoxylin was used to briefly stain them. The fixed sections were quickly used for laser capture micro-dissection with the UV laser-based PALM MicroBeam system (P.A.L.M. Microlaser Technologies AG, Bernried, Germany). Once the 
cell areas were chosen microscopically, the PALM RoboSoftware controlling a pulsed 337 $\mathrm{nm}$ UV-laser automatically cut around the selected area and then transferred it to a collection tube. A $500 \mu 1$ microcentrifuge tube cap was filled with $20 \mu \mathrm{l}$ of mineral oil for sample collection for the RNA analysis. The collected material was deposited at $-80^{\circ} \mathrm{C}$ for RNA isolation.

\subsection{Molecular biology techniques}

\subsubsection{Microarray analysis of irradiated liver}

\subsubsection{1 cDNA generation and performance of microarray}

Utilising the facility of Georg August-University (Transkriptom analysis lab., Goettingen, Germany), microarray analysis was performed. After 6 hours of irradiation to rat and sham-irradiated controls, the cDNA was generated from the isolated liver RNA $(1 \mu \mathrm{g})$.

The cDNA preparation was performed by using Super-Script Choice System (Invitrogen, Karlsruhe, Germany) as instructed. Using the BIOARRAY HIGHYIELD® RNA transcript labeling kit (Affymetrix, Santa Clara, CA, USA) biotin-labeled cRNA was prepared according to company instruction and incorporated nucleotides were separated from unincorporated nucleotides with the help of RNeasy columns (Qiagen, Hilden, Germany) according to Harnacke et al. (2005). A Test 2 Array (Affymetrix, Santa Clara, CA, USA) was used to test the RNA as instructed.

Then it was loaded onto the Affymetrix GeneChip Rat (Affymetrix, Santa Clara, CA, USA) following the manufacturer's instructions to accomplish hybridisation with the probes. Thereafter, the chip was washed and stained in the Affymetrix Fluidics Station. The washing steps were done using 6x SSPE-T $\left(10\right.$ times, $\left.25^{\circ} \mathrm{C}\right)$ and $0.5 \mathrm{x}$ SSPE-T (4 times, $50^{\circ} \mathrm{C}$ ). A $2 \mathrm{~g} / 1$ streptavidin-phycoerythrin conjugate (Molecular Probes, Eugene, Oregon, USA) was used to stain the biotinylated cRNA $\left(30 \mathrm{~min} ., 25^{\circ} \mathrm{C}\right)$, followed by washing with $6 \mathrm{x}$ SSPE-T at $25^{\circ} \mathrm{C}$. Additionally, the signal was amplified by employing a $0.1 \mathrm{mg} / \mathrm{ml}$ normal goat IgG (Sigma, Munich, Germany) solution and a $3 \mathrm{mg} / \mathrm{l}$ biotinylated anti-streptavidin goat antibody (Vector Laboratories, Burlingame, California, USA) solution, followed by a 30 minutes staining step at $25^{\circ} \mathrm{C}(2 \mathrm{~g} / 1$ streptavidin-phycoerythrin conjugate in $6 \mathrm{x}$ SSPE-T $)$ and washing with 6 x SSPE-T (10 times, $\left.25^{\circ} \mathrm{C}\right)$.

Scanning of the array was performed utilising a confocal-laser scanning microscope with a wavelength at $560 \mathrm{~nm}$ by G2500A Gene-array Scanner from Hewlett-Packard (Bad 
Homburg vor der Höhe, Germany). Affymetrix Suite version 4.0 Software was used for analysing quantitative scanning readings as described by Ebbesen et al. (2006).

\subsubsection{Data analysis}

Utilising the Bioconductor (https://www.bioconductor.org) and its respective packages (Affy and Limma), microarray data was analysed as described by Gentleman et al. (2004) and Smyth (2004). The following steps were involved in the microarray data analysis: a. Robust Multichip Analysis (RMA) preprocessing b. global clustering and principal component analysis c. adaption of data to a linear model d. determination of differential gene expression. The background correction and probe summary was included in the normalisation process which was done by using the RMA approach according to Irizarry et al. (2003). The calculated linear relation of variables was expressed as 1-Pearson's Correlation Coefficient. Utilising princomp-function in the R software, principal component analysis was performed.

The data was adapted to simple linear model in order to evaluate the average values for every gene and quantification of differences in the gene expression level. Using this for every gene, the group's median and standard deviations were calculated. The data was further evaluated by the empirical Bayesian statistics to identify those genes which underwent significant changes in expression between the groups.

This was done by controlling the standard errors of the calculated values as described before by Ortiz-Pineda et al. (2009) and Smyth (2004). The p-values from t-test were corrected for multiple testing by the Benjamini-Hochberg method Benjamini et al. (2001). Adjustment of p-value made sure to minimise false-positives through adjusting the false discovery rate (FDR). In case FDR of a gene was less than 0.05 , the null hypothesis was rejected. That would mean that no difference in gene expression between degradation levels was considered.

\subsubsection{Generation and amplification of $c D N A$}

\subsubsection{Real time-PCR}

RT-PCR was done based on the laboratory protocol described by Sheikh (2006). First cDNA was produced, the cDNA-generating system consisted of $1 \mu \mathrm{g}$ mRNA, dNTPs (100 $\mathrm{nM})$, oligo(dT)15 primer (50 pM), reverse transcriptase from murine moloney leukemia virus (M-MLV RT; $200 \mathrm{U}$ ), RNase inhibitor (16 U), 1x RT buffer, $2.5 \mu \mathrm{l}$ DTT (100 mM). The samples were vortexed (Schütt Labortechnik, Göttingen, Germany) and incubation was 
carried out at $40^{\circ} \mathrm{C}$ for 60 minutes. The expressions of CXCL1, CXCL2, CXCL5, CXCL8, CXCL10, CCL2, CCL7, IL-1beta, IL-6, IFN-gamma, CXCR-2, CCR2, platelet/endothelial cell adhesion molecule (PECAM-1) and neutrophil elastase genes were analysed employing qPCR SuperMix-UDG (Platinum ${ }^{\circledR}$ SYBR ${ }^{\circledR}-$ Green) which was purchased from Invitrogen, (Karlsruhe, Germany). The UBC and beta-actin were utilised as housekeeping genes. Table 1 shows the primer sequences used.

Analysis of the cDNA samples was done and RT-PCR reaction was performed by using the following recipe:

Volume for one reaction

SYBR Green Master Mix

$13.0 \mu 1$

Primer-sense $(5 \mathrm{mM})$ " $\mathrm{X}$ "

$1.5 \mu 1$

Primer-anti-sense $(5 \mathrm{mM})$ " $\mathrm{X}$ ”

$1.5 \mu 1$

$\mathrm{H}_{2} \mathrm{O}$ (Ampuwa $\left.{ }^{\circledR}\right)$

$6.5 \mu 1$

Amount of templete used per reaction was $2.5 \mu \mathrm{l}$ cDNA and the same amount of water was provided in negative control.

Table 1: RT-PCR primers list and sequences

\begin{tabular}{|c|c|c|}
\hline Primer & $\begin{array}{c}5 \rightarrow \mathbf{3} \\
\text { Sense }\end{array}$ & $\begin{array}{c}5 \rightarrow \mathbf{3} \\
\text { Anti-sense }\end{array}$ \\
\hline MIP-2/CXCL2 & ATCCAGAGCTTGACGGTGAC & AGGTACGATCCAGGCTTCCT \\
\hline CXCR2 & CCAAGCTGATCAAGGAGACC & GGGGTTAAGACAGCTGTGGA \\
\hline LIX/CXCL5 & CTCAAGCTGCTCCTTTCTCG & GCGATCATTTTGGGGTTAAT \\
\hline KC/CXCL1 & GGCAGGGATTCACTTCAAGA & GCCATCGGTGCAATCTATCT \\
\hline MCP-1/CCL2 & AGGCAGATGCAGTTAATGCCC & ACACCTGCTGCTGGTGATTCTC \\
\hline MCP-3/CCL7 & GCATGGAAGTCTGTGCTGAA & CCGTTCCTACCCCTTAGGAC \\
\hline CCR2 & CTTGTGGCCCTTATTTTCCA & AGATGAGCCTCACAGCCCTA \\
\hline NE & CTTTGAGAACGGCTTTGACC & CACATTGAGCTCTTGGAGCA \\
\hline PECAM-1 & TCAGCTGCCAGTCAGTAAATGG & TCTGGAAGTTGCTCTTTGCTCTT \\
\hline IL-8/CXCL8 & CCCCCATGGTTCAGAAGATTG & TTGTCAGAAGCCAGCGTTCAC \\
\hline IFN-gamma & GAACTGGCAAAAGGACGGTA & CTGATGGCCTGGTTGTCTTT \\
\hline IP-10/CXCL10 & CTGTCGTTCTCT GCCTCGTG & GGATCCCTTGAGTCCCACTCA \\
\hline
\end{tabular}




\begin{tabular}{|c|c|c|}
\hline IL-1beta & TACCTATGTCTTGCCCGTGGAG & ATCATCCCACGAGTCACAGAGG \\
\hline IL-6 & GTCAACTCCATCTGCCCTTCAG & GGCAGTGGCTGTCAACAACAT \\
\hline$\beta$-actin & ACCACCATGTACCCAGGCATT & CCACACAGAGTACTTGCGCTCA \\
\hline UBC & CACCAAGAAGGTCAAACAGGAA & AAGACACCTCCCCATCAAACC \\
\hline
\end{tabular}

\subsubsection{Thermal cycler amplification program}

With the help of the ABI prism 7000 sequence detection system by Applied Biosystems (Foster City, California, USA), the PCR was performed by using following program with 45 cylces: 120 seconds at $50^{\circ} \mathrm{C}, 120$ seconds at $95^{\circ} \mathrm{C}, 15$ seconds at $95^{\circ} \mathrm{C}, 30$ seconds at $60^{\circ} \mathrm{C}$. Assay of all the samples was done in duplicate. Using the Platinum SYBR Green qPCR mix UDG, the expressions of the different genes were analysed. PCR-product specificity was controlled using the dissociation curve of the PCR amplification program and the $\mathrm{Ct}$ values were obtained by evaluating the amplification curves. Primer express software was used to determine the specific dissociation temperature. The normalisation of the data was achieved comparing with housekeeping genes by applying the Graphpad Prism 4 software (Graphpad, San Diego, USA). Using the Ct values the fold change expression was calculated.

\subsubsection{Primer designing}

Primer designing was performed by utilising the data bank of genes (http://www.ncbi.nlm.nih.gov) and program "Primer express", primers were designed for different genes.

\subsubsection{Extraction of total RNA}

\subsubsection{RNA extraction procedure}

The mRNA was isolated by utilising NucleoSpin ${ }^{\circledR}$ RNAII kit (Macherey-Nagel, Düren, Germany) from cultured hepatocytes, myofibroblasts and laser-microdissected liver samples of rat by employing the instruction provided by company.

\subsubsection{RNA extraction by density-gradient ultracentrifugation}

Total hepatic RNA was recovered according to Chirgwin et al. (1979): extraction by guanidine isothiocyanate, ultracentrifugation by using Centricon T-2070 ultracentrifuge, 
(Kontron Instruments, Neufahrn, Germany) in a $\mathrm{CsCl}$ density gradient, precipitation by ethanol.

The precipitated RNA was centrifuged $\left(12,000 \mathrm{rpm} 4^{\circ} \mathrm{C}, 0.5 \mathrm{~h}\right.$; Eppendorf, MiniSpin 5415C, Hamburg, Germany) as described by Sheikh (2006) and Tron (2004) to collect a pellet, which was washed, after discarding the supernate, in $0.2 \mathrm{ml}$ ice-cold $70 \%$ ethanol, to remove residual Na-acetate. RNase-free water $(100 \mu \mathrm{l})$ was used to reconstitute the pellets. Utilising a GeneQuant II photometer (Pharmacia Biotech, Freiburg, Germany), RNA concentration and purity was assessed at two different wavelengths (260 and $280 \mathrm{~nm})$ after diluting (1:100) one aliquot of the RNA sample in RNase-free water.

Solutions used for ultracentrifugation: After having been sterile filtered, the guanidine isothiocyanate (GITC) buffer (4 M guanidine isothiocyanate, $25 \mathrm{mM} 0.25 \mathrm{M}$ sodium citrate, $0.5 \%$ N-lauroylsarcosyl) was stored in the dark at $4^{\circ} \mathrm{C}$. Just before usage the $\beta$ mercaptoethanol was added in the ratio of 1 to $100 \mu \mathrm{l}$ of the GITC buffer. The $\mathrm{pH}$ of the cesium chloride $(\mathrm{CsCl})$ buffer $(5.7 \mathrm{M}$ cesium chloride, $25 \mathrm{mM} 250 \mathrm{mM}$ sodium citrate, 100 $\mathrm{mM} 500 \mathrm{mM}$ EDTA) was adjusted to 7.5 by adding $250 \mathrm{mM}$ citric acid; and then dissolved in RNase-free $\mathrm{H}_{2} \mathrm{O}$, after which it was sterile-filtered and kept prior to use as described by Sheikh (2006) and Tron (2004).

\subsubsection{Northern blot}

The experimental apparatus was made free of RNase by placing it 10-20 minutes in $0.1 \%$ DEPC solution. RNA processing and Northern-blot analysis was performed as described previously by Sheikh (2006) and Tron (2004).

\subsubsection{Processing of RNA samples for further analyses}

5-10 ug of RNA was dissolved in RNase-free $\mathrm{H}_{2} \mathrm{O}(5 \mu \mathrm{l})$. Afterwards, $7.5 \mu 1$ sample buffer was added and volume was reduced to $5 \mu$ by using savant ${ }^{\mathrm{TM}}$ SpeedVac vacuum centrifuge (Thermo Life Sciences, Egelsbach, Germany). Next, the samples were denatured by heating $\left(10 \mathrm{~min}\right.$ at $\left.65^{\circ} \mathrm{C}\right)$ and shortly cooling them by ice (ice-machine, Ziegra, Isernhagen, Germany) followed by centrifugation (Eppendorf minispin centrifuge; 10,000 rpm, 60 seconds). Every sample obtained loading buffer $(3 \mu \mathrm{l})$ and then was centrifuged using above mentioned conditions as described by Sheikh (2006) and Tron (2004). 


\subsubsection{Electrophoresis}

A $1 \%$ agarose gel was prepared using agarose dissolved in $1 \mathrm{x}$ TAE buffer by warming (Microwave oven, Siemens, Munich, Germany). Electrophoresis was executed by using MiniProtean ${ }^{\circledR}$ III electrophoresis system (Bio-Rad, Munich, Germany) for 60-90 minutes at a constant voltage $(80 \mathrm{~V})$ provided by Power Pac 300 power supply from Bio-Rad, (Munich, Germany) and thus, the quality of RNA was assessed by using the built-in UV transilluminator of the Eagle Eye ${ }^{\mathrm{TM}}$ system (Stratagene Europe, Amsterdam, Netherlands); after photographing the gel, it was rapidly subjected to blotting.

\subsubsection{RNA transfer on membrane}

RNA was transferred to a membrane (nylon) after it was separated on the gel by utilising capillary transfer method. 0.51 of $20 \mathrm{x}$ SSC was poured into the tray (plastic). Whatman paper $(3 \mathrm{~mm})$ filter was slightly dipped in this solution and swatch-over-glass plate which was positioned in a way over tray that both ends remained soaked in buffer to work as wick. The bubbles were removed from the filter and gel was positioned towards upside down on Whatman paper and the bubbles were smoothed out. Next, 2x SSC soaked nylon membrane was put on the upper side of gel. Afterwards, the membrane was covered with $2 \mathrm{x}$ SSC-wetted filter paper ( 2 pieces) and made bubbles-free. Then, towels were shielded with second glass plate and $1000 \mathrm{~g}$ weight was put on top and left overnight for RNA transfer. Then, UV crosslinking for 2 minutes from both sides was performed, the RNA was fixed on the membrane using the Stratalinker ${ }^{\mathrm{TM}} 180$ (Stratagene, Heidelberg, Germany) set in "autocross link" mode.

Prior to hybridisation, the membrane was transferred to a hybridisation tube, thereby carefully avoiding bubbles. Pre-hybridisation was performed using the hybridisation solution $\left(10 \mathrm{ml}\right.$; QuikHyb ${ }^{\circledR}$ Stratagene, TX, USA, $\left.68^{\circ} \mathrm{C}\right)$ for 120 minutes in hybridisation oven (Biometra, Göttingen, Germany).

After labelling the DNA probe by Nick-translation method, it was cleaned by passing through Sephadex G-50 columns and utilising liquid scintillation counter (Wallac 1409, Turku, Finland), the radioactivity was calculated as described previously by Sheikh (2006). For obtaining a suitable volume of cDNA (radiolabeled), the respective amount of QuikHyb® solution was determined:

Vol. of ${ }^{32}$ phosphate $\mathrm{cDNA}=$ vol. of quikhyb $\mathrm{x} 1 * 10^{6} /$ beta-radioactivity of cDNA $(1 \mu 1)$ 
Vol. of DNA (salmon sperm) $=2 \times$ Vol. of ${ }^{32}$ phosphate cDNA

Time-dependent changes of radioactivity were calculated according to following formula:

beta-radioactivity of cDNA $(1 \mu \mathrm{l}) \times \mathrm{Y}=\mathrm{Z}$ counts per minute $/ \mu 1$

$$
\mathrm{Y}=\text { decay correction value of }{ }^{32} \mathrm{P}
$$

\subsubsection{4. $c D N A / R N A$ hybridisation}

Following the crosslinking, 1x TE buffer was used to wash the membrane (nylon) to remove all traces of agarose. The radiolabeled probe was combined with the double amount of DNA from salmon sperm. Nucleic acids were denatured $\left(95^{\circ} \mathrm{C}\right)$ for 5 minutes, and thereafter chilled-down for 3-5 minutes on ice. After briefly centrifuging the samples, they were transferred into the hybridisation tube containing QuikHyb ${ }^{\circledR}$ solution and then kept in hybridisation oven $\left(68^{\circ} \mathrm{C}, 120 \mathrm{~min}\right)$.

Washing: When the hybridisation was complete, the membrane was rinsed with $2 \mathrm{x}$ $\mathrm{SSC} / 0.1 \% \mathrm{SDS}(30 \mathrm{ml})$ in the tube. Using same solution, the membrane was rinsed again for 10 minutes at r.t. Afterwards, the membrane was washed at $55^{\circ} \mathrm{C}$ in the hybridisation oven by using $1 \mathrm{x}$ SSC/0.1\% SDS and $2 \mathrm{x}$ SSC/0.1\% SDS two times each, respectively. After radioactivity measurements revealed values not higher than $100 \mathrm{cpm}$, the washing steps were halted. Thereafter, an X-ray film cassette $(10 \times 18)$ from Siemens (Erlangen, Germany) was used to place the cling film packed membrane. The membrane was exposed to X-ray film and developed by using Konica (Hohenbrunn, Germany) SRX-101A developing device.

\subsubsection{Solutions}

The running buffer was prepared in a 10x stock (200 mM MOPS, $50 \mathrm{mM} 2 \mathrm{M}$ sodium acetate, $10 \mathrm{mM} 0.5 \mathrm{M}$ EDTA). The $\mathrm{pH}$ was set to 7.0 using $5 \mathrm{~N} \mathrm{NaOH}$, and afterwards the solution was deposited at $4{ }^{\circ} \mathrm{C}$ in the dark after sterile filtration. The sample buffer solution containing deionised formamide $(0.5 \mathrm{ml}), 37 \%$ formaldehyde $(0.169 \mathrm{ml})$, and $10 \mathrm{x}$ running buffer $(0.1 \mathrm{ml})$ was divided and kept $\left(-20^{\circ} \mathrm{C}\right)$ after portioning it into $1000 \mu \mathrm{l}$ volume for two to three months. Deionised formamide was prepared by adding $10 \mathrm{~g}$ mixed bed resin AG 501$\mathrm{X} 8$ (D) to 0.11 formamide. The solution was kept at r.t. and stirred for $0.5 \mathrm{~h}$ in the dark followed by sterile filtering, distributed in $50 \mathrm{ml}$ portions, deposited at $-20^{\circ} \mathrm{C}$ in dark. The loading buffer $\left(5 \mathrm{ml}\right.$ of 10x RB, $3 \mathrm{ml}$ RNase-free Ampuwa ${ }^{\circledR}$ water, $1.5 \mathrm{~g}$ of Ficoll 400, 10 $\mathrm{mg}$ of bromophenol blue) was distributed into $500 \mu \mathrm{lml}$ portions and deposited $\left(-20^{\circ} \mathrm{C}\right)$ for 
two to three months. A 20x SSC solution $\left(350.6 \mathrm{~g} \mathrm{NaCl}, 176.4 \mathrm{~g}\right.$ sodium citrate $\times 2 \mathrm{H}_{2} \mathrm{O}$ in 11 ) was autoclaved and stored prior to use.

\subsubsection{DNA amplification by polymerase chain reaction (PCR)}

Procedure of the PCR and agarose gel electrophoresis of the cDNA including all the solutions was done incorporating the standard laboratory protocol recommended by Sheikh (2006). As a result of the polymerase chain reaction using Thermocycler, (Mastercycler ${ }^{\circledR}$ gradient, Eppendorf, Hamburg, Germany), amplification of the fragments was possible due to the repeated cycles of cDNA synthesis. Amplification of rat CXCL1 and $\beta$-actin gene was done by utilising specific sense and anti-sense primers and Go Taq Green Master mix. Below is the recipe for each reaction:

Go Taq ${ }^{\circledR}$ Green Master mix composition

$\begin{array}{ll}\text { dCTP } & 400 \mu \mathrm{M} \\ \text { dATP } & 400 \mu \mathrm{M} \\ \text { dTTP } & 400 \mu \mathrm{M} \\ \text { dGTP } & 400 \mu \mathrm{M} \\ \mathrm{MgCl}_{2} & 3 \mathrm{mM} \\ \text { dATP } & 400 \mu \mathrm{M}\end{array}$

\begin{tabular}{ll}
\multicolumn{2}{c}{ PCR mix } \\
Master mix & $12.5 \mu \mathrm{l}$ \\
Sense primer (5 pmol) & $1.5 \mu 1$ \\
Anti-sense primer (5 pmol) & $1.5 \mu 1$ \\
$\mathrm{H}_{2} \mathrm{O}$ add to final amount & $7.5 \mu 1$ \\
cDNA & $2.5 \mu 1$
\end{tabular}

Next, the product size has been assessed through electrophoresis.

Table 2: Northern Blot sense and anti-sense primer sequences

\begin{tabular}{|l|l|l|}
\multicolumn{2}{c}{ Sense $\quad 5^{\prime} \longrightarrow 3^{\prime}$} & Anti-sense 5' $\longrightarrow$ 3' \\
\hline KC/CXCL1 & GGCAGGGATTCACTTCAAGA & GCCATCGGTGCAATCTATCT \\
& & \\
\hline$\beta$-actin & ACCACCATGTACCCAGGCATT & CCACACAGAGTACTTGCGCTCA \\
\hline
\end{tabular}

\subsubsection{DNA amplification by Agarose gel electrophoresis}

After amplification of cDNA, an electrophoresis was done in 1\% agarose to detect the specific probe as described by Sheikh (2006). This was performed by pipetting (Type Reference, Eppendorf, Hamburg, Germany) the $3 \mu 1$ of loading buffer (6x) together with $10 \mu 1$ of PCR product along GeneRuler TM 100bp DNA ladder plus, and electrophoresis was 
performed for 60 minutes $(80 \mathrm{~V})$. Next, DNA strands with inserted ethidium bromide were recognised by ultraviolet light at $312 \mathrm{~nm}$ (Ultravioletemitter, Bachofer, Reutlingen, Germany). The 20x Tris/acetate/EDTA (TAE) buffer was prepared by combining $1 \mathrm{M}$ Tris base, $0.4 \mathrm{M}$ sodium acetate, and $40 \mathrm{mM}$ EDTA. Using acetic acid $7.4 \mathrm{pH}$ was maintained, once the EDTA was fully suspended; before storing at r.t. the buffer was autoclaved.

\subsection{Biochemical methods}

\subsubsection{Protein extraction from liver tissue}

Preparation of tissue lysates: The procedures for the protein isolation and Western blot analysis have been executed in accordance with standard laboratory protocol as described by Tron (2004) and Zibrova (2004) incorporating slight modifications.

To avoid the proteolytic degradation of the proteins, the whole experiment was conducted at $4^{\circ} \mathrm{C}$. The tissue sample $(100 \mathrm{mg}$ each) was homogenised by using Ultra-Turrax TP-18 10S1 which was purchased from Janke and Kunkel (Staufen, Germany). It was repeated thrice ( $10 \mathrm{sec}$. every time), in 10 volumes of $50 \mathrm{mM}$ Tris-HCl buffer at $\mathrm{pH} 7.4$. The buffer contained $150 \mathrm{mM} \mathrm{NaCl}, 1 \mathrm{mM}$ EDTA, 1\% Triton X-100, 1mM PMSF, 1mM benzamidine, $1 \mu \mathrm{g} / 1000 \mu \mathrm{l}$ antipain, $1 \mu \mathrm{g} / 1000 \mu \mathrm{l}$ leupeptin, $1 \mu \mathrm{g} / 1000 \mu \mathrm{l}$ pepstatin A and $10 \mu \mathrm{M}$ chymostatin. The homogenates were processed 5 times by using a $22 \mathrm{G}$ injection cannula which was attached to a syringe. By centrifugation, the supernatant containing the proteins was separated from cell debris $\left(5 \mathrm{~min}, 10,000 \mathrm{~g}, 4^{\circ} \mathrm{C}\right)$ thereafter. Utilising a BCA protein assay reagent kit (Pierce, Bonn, Germany), the protein concentration of the supernatant have been measured using the method of Smith et al. (1985). The stock was portioned and stored until usage $\left(-20^{\circ} \mathrm{C}\right)$.

The solutions were prepared according to the standard lab recipes described by Tron (2004). A 10x homogenisation buffer ( $\mathrm{pH} 7.4$ ) consisting of Tris- $\mathrm{HCl}$ (50 mM of $2 \mathrm{M}$ stock), EDTA (1 mM of $0.5 \mathrm{M}$ stock) and $\mathrm{NaCl}(150 \mathrm{mM})$ for tissue processing and a $1 \mathrm{x}$ homogenisation buffer with additives (10x homogenisation buffer, 1\% Triton X-100) was prepared. Just before use the protease inhibitors (500 mM PMSF, $10 \mathrm{mg} / \mathrm{ml}$ leupeptin, $1 \mathrm{M}$ benzamidine, $8.25 \mathrm{mM}$ chymostatin, $1 \mathrm{mg} / \mathrm{ml}$ pepstatin, $10 \mathrm{mg} / \mathrm{ml}$ antipain) were added. 


\subsubsection{Western blot analysis}

\subsubsection{Sample preparation}

The aliquots containing the prepared tissue homogenates were denatured in samplebuffer via boiling $\left(95^{\circ} \mathrm{C}, 10 \mathrm{~min}\right)$. The buffer comprised $2 \%$ SDS, $10 \%$ glycerol, $50 \mu \mathrm{g} / \mathrm{ml}$ bromophenol blue, $2 \%$ beta-mercaptoethanol and $50 \mathrm{mM}$ Tris- $\mathrm{HCl}$ at $\mathrm{pH}$ 6.8. Next, SDSpolyacrylamide gel electrophoresis (SDS-PAGE) was performed by using $15 \mu \mathrm{g}$ of the total protein.

\subsubsection{SDS-polyacrylamide gel}

For all applications mentioned above, a $12.5 \%$ separating Tris/ glycine SDS polyacrylamide ready-made gel (SDS-PAGE) from (Invitrogen, Darmstadt, Germany) was used following the company's guidelines. The Western blot test based on Laemmli's method (Laemmli, 1970) was done.

\subsubsection{SDS-polyacrylamide gel electrophoresis (SDS-PAGE) and electrophoretic transfer}

The samples were transferred to the bottom of the wells and electrophoresis was run at a constant $20 \mathrm{~mA}$ per gel. The Rainbow ${ }^{\mathrm{TM}}$ colored protein markers (Amersham Pharmacia Biotech, Freiburg, Germany) were employed as molecular weight standards. The electrophoretic transfers were performed using the procedure described by Towbin et al. (1979). Fiber pads, filter paper and nitrocellulose transfer membrane $(0.45 \mu \mathrm{M}$ pore size $)$ were soaked in the transfer buffer, just before halting the gel running. The gel was removed from the plates after completion of the electrophoresis and immersed in the transfer buffer. Utilising a Mini-Trans-Blot ${ }^{\circledR}$ Cell (Bio-Rad, Munich, Germany), proteins were transferred onto the membrane from gel. According to guidelines from Bio-Rad the transblot sandwich was assembled. The transblot sandwich thus assembled was inserted into the transblot cell filled with the transfer buffer. Just behind the cathode side of the transblot cell, the ice-cooling unit was set up. The transfer was run for 2 hours at $350 \mathrm{~mA}$ with only one change in the icecooling unit after the first hour.

\subsubsection{Immunovisualisation}

Post transfer, the membrane was incubated overnight $\left(4^{\circ} \mathrm{C}\right)$ in the blocking solution and thereafter the incubation of the membrane was carried out with diluted primary antibody for 120 minutes at r.t. After six washes, (each five minutes), the membrane was then 
incubated with the HRP-conjugated secondary antibody diluted in the antibody dilution buffer for 60 minutes at r.t. Afterwards, the membrane was rinsed, as done earlier. SuperSignal ${ }^{\circledR}$ West Pico Chemiluminescent Substrate (Pierce, Bonn, Germany) was used to detect the chemiluminescence. Equal volumes of both the substrate components were mixed to prepare the substrate working solution. The membrane was then incubated with the substrate working solution $(5 \mathrm{~min})$ at r.t., and fitted between two sheets of transparent plastic protector. The membrane was then exposed to X-ray film and developed thereafter.

Samples and loading buffer were used per instructions by Invitrogen.

Blocking reagent

$$
\text { For } 50 \mathrm{ml} \quad \text { Final concentration }
$$

Nonfat dry milk

$2.5 \mathrm{~g} \quad 5 \%$

The solution must be freshly prepared before storing at $4{ }^{\circ} \mathrm{C}$.

Antibody incubation buffer

$\begin{array}{lll} & \text { For } 50 \mathrm{ml} & \text { Final concentration } \\ 5 \% \text { nonfat milk } & 5 \mathrm{ml} & 0.5 \%\end{array}$

Primary antibodies were utilised in the dilutions given below:

Antibody

Used dilution

anti-CXCL1 rabbit polyclonal antiserum

1:1000

anti- $\beta$-actin mouse monoclonal antibody

$1: 5000$

Secondary HRP-conjugated antibodies were used in the dilutions given below:

Antibody Used dilution

Donkey anti-rabbit whole Ig $1: 2000$

Rabbit anti-mouse Ig 1:2000

\subsubsection{Enzyme-Linked Immunosorbent Assay (ELISA)}

The ELISA procedure including all the solutions was done based on manufacturer's instructions. In order to measure the MIP-2/CXCL2 concentration in rat serum, the Quantikine ${ }^{\circledR}$ M rat CXCL2 immunoassay kit (R\&D Systems, Wiesbaden, Germany), was used, following company's instruction. All the reagents were prepared short before the experiment was started. The kit contained all the reagents including experimental control.

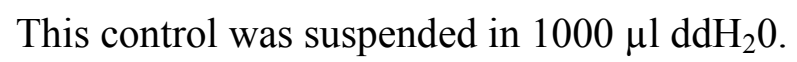




\subsubsection{Substrate reagents}

The Quantikine ${ }^{\circledR}$ M kit contained color reagents named as A and B which were put together in equal volumes prior to use.

\subsubsection{Probes, standards and measurements}

Standards and probes were prepared and measured according to the company's instruction. The serum probes were diluted 2 times before use and $50 \mu \mathrm{l}$ from this dilution was pipetted to each well. All the standards and probes were determined in duplicate including control.

\subsection{Clinical chemistry methods}

\subsubsection{Measurement of enzymes in serum}

Aminotransferases ("transaminases") are commonly used in clinic to detect liver damage. Mostly, alanine and aspartate aminotransferases ("ALT" and "AST") are measured for this purpose. Additional determinations of alkaline phosphatase (AP) are recommendable. These enzymes were measured by employing the DiaSys kit (Diagnostic Systems International, Holzheim Germany) in the facility of the clinic.

\subsubsection{Statistical Analysis}

Utilising Graphpad Prism 4 software (Graphpad, San Diego, USA) the data was analysed to determine S.E.M values, Student's t-test, One-way ANOVA and Dunnett post hoc test. $P<0.05$ value was considered significant.

\subsubsection{Safety Measures}

All the procedures that dealt with plasmid DNA and genetically modified organisms were performed by following the instructions mentioned in "Gentechnikgesetz" and "Gentechnik Sicherheitordnung" both issued in 1990. Environmentally harmful chemicals such as DEPC, formaldehayde and ethidium bromide employed in course of this study, were meticulously managed and carefully disposed maintaining the rules of the institute. Experiments using radionuclides were carried out in radioactivity level II laboratories. The use of radiochemicals and discarding of radioactive waste were carried out according to the guidelines of institution. 


\section{RESULTS}

\subsection{The effect of radiation on the serum levels of liver enzymes and macrophage inflammatory protein-2/CXCL2}

To investigate liver damage following irradiation, levels of aminotransferases and the pro-inflammatory chemokine MIP/CXCL2 were determined in serum samples from irradiated and sham-irradiated animals. Measurements of alkaline phosphatase (AP) and hepatic alanine aminotransferase (ALT) and aspartate aminotransferase (AST) are part of the standard clinical test panel. The two aminotransferases, in particular, are the most reliable markers of hepatocellular injury or necrosis.

A slight elevation in the serum AP level was observed 1 hour after irradiation (Fig. 5a). This was followed by a return to the baseline level 6 hours after irradiation, and no

a)
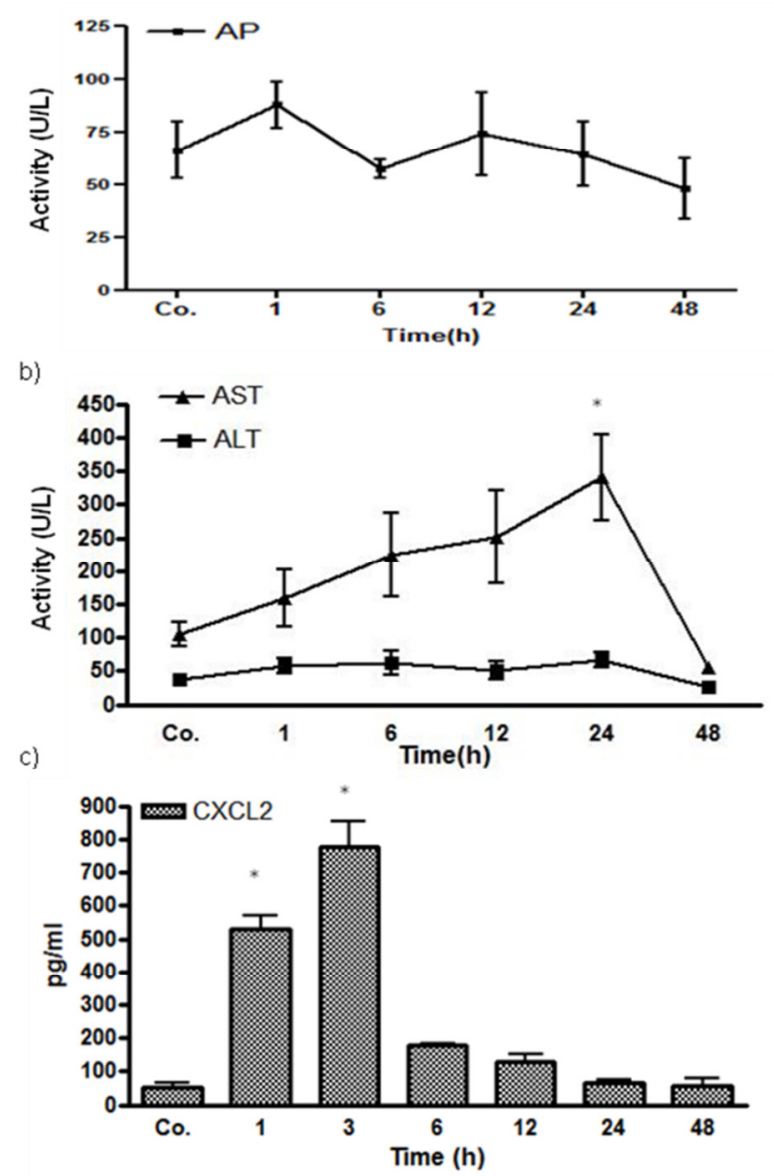

Figure 5: Serum concentrations of liver enzymes and of macrophage inflammatory protein-2 (MIP-2)/CXCL2 in rats following liver irradiation. (a) The serum alkaline phosphatase level rose 1 hour after irradiation and it declined thereafter. (b) The serum alanine aminotransferase level was slightly elevated for up to 24 hours after 
irradiation, while the serum aspartate aminotransferase (AST) level increased continuously for up to 24 hours after irradiation. The serum AST level increased significantly to its maximum level 24 hours after irradiation in comparison with control rats. (c) Serum levels of the MIP-2/CXCL2 protein in the irradiated and control rats. The serum concentration of MIP-2/CXCL2 was measured using an enzyme-linked immunosorbent assay. The increase in the serum concentration of MIP-2/CXCL2 between 1 hour and 3 hours after irradiation was statistically significant. Results are representative of 3 -experiments. A value of $P<0.05$ was considered statistically significant. The data presented are the means \pm standard errors of the means.

Abbreviations: AP, alkaline phosphatase; ALT, alanine aminotransferase; AST, aspartate aminotransferase; Co, control.

changes were observed subsequently. The serum AST level rose 1 hour after irradiation and it peaked at 24 hours after irradiation. The analysis of the data using Student's t-test determined that the increase in the serum AST level at 24 hours after irradiation was statistically significant. The serum ALT level did not change throughout the experiment (Fig. 5b).

Compared with the control rats, a significant early rise in the serum MIP-2/CXCL2 level was detected between 1 hour and 3 hours after irradiation. The maximum serum MIP2/CXCL2 level of up to $774 \pm 80 \mathrm{pg} / \mathrm{ml}$ was detected 3 hours after irradiation, and it declined rapidly thereafter $(P<0.05)$ (Fig. 5c). Hence, gamma irradiation revealed significant alteration in AST and pro-inflammatory marker MIP-2/CXCL2 levels in the serum.

\subsection{Immunohistochemical detection of leukocytes in the irradiated rat liver}

\subsubsection{Detection of neutrophils after rat liver irradiation}

Neutrophils secrete neutrophil elastase (NE) during inflammation (Takahashi et al. 1988). To investigate tissue inflammation following irradiation, the liver tissues from irradiated and sham-irradiated control rats were stained to detect the presence of NE and additional markers. Immunohistological staining for NE showed the presence of neutrophils in the portal vessels of the liver following irradiation compared with the sham-irradiated control animals (Fig. 6a \& b). Counting the $\mathrm{NE}^{+}$cells determined that there was an early accumulation of $\mathrm{NE}^{+}$neutrophils around the portal vessels of the hepatic liver 1 hour postirradiation, and that the mean number of cells peaked at 6 hours after irradiation $(60.2 \pm 16.8$ cells), an increase that was statistically significant. At 12 hours and 24 hours after irradiation the numbers of $\mathrm{NE}^{+}$cells had declined (Fig. 6c). The parenchymal and central areas of the irradiated and control livers contained few $\mathrm{NE}^{+}$cells. Reverse transcriptase-polymerase chain reaction (RT-PCR) analysis detected a $7.2 \pm 2.3$-fold increase in the expression of NE messenger ribonucleic acid (RNA) at 6 hours (Fig. 6d). 


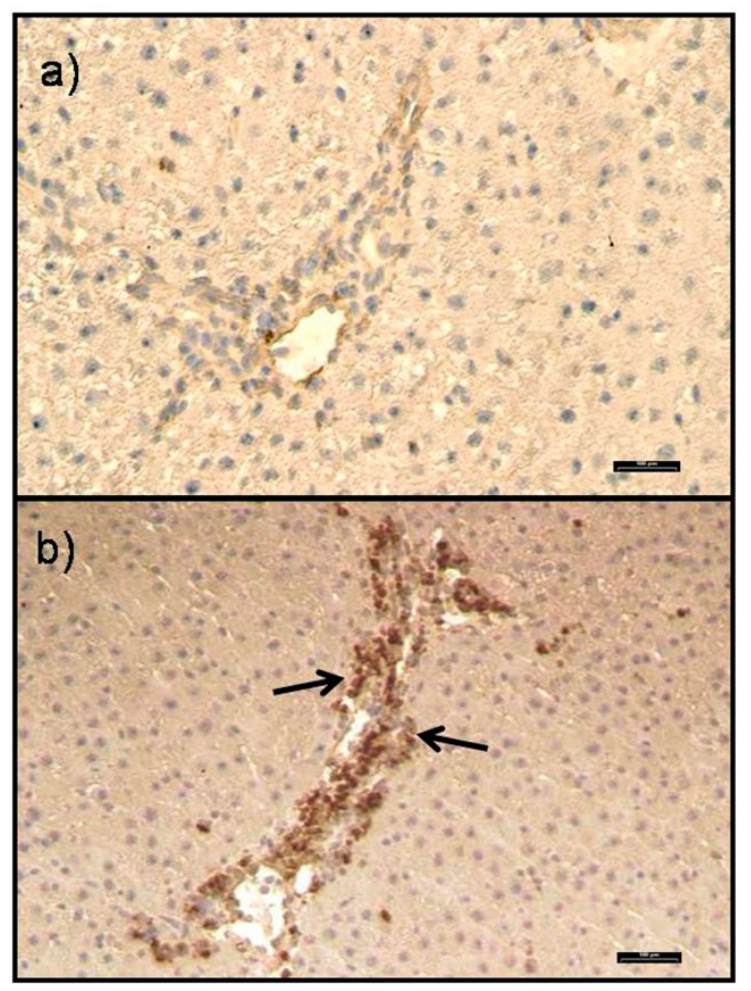

c)
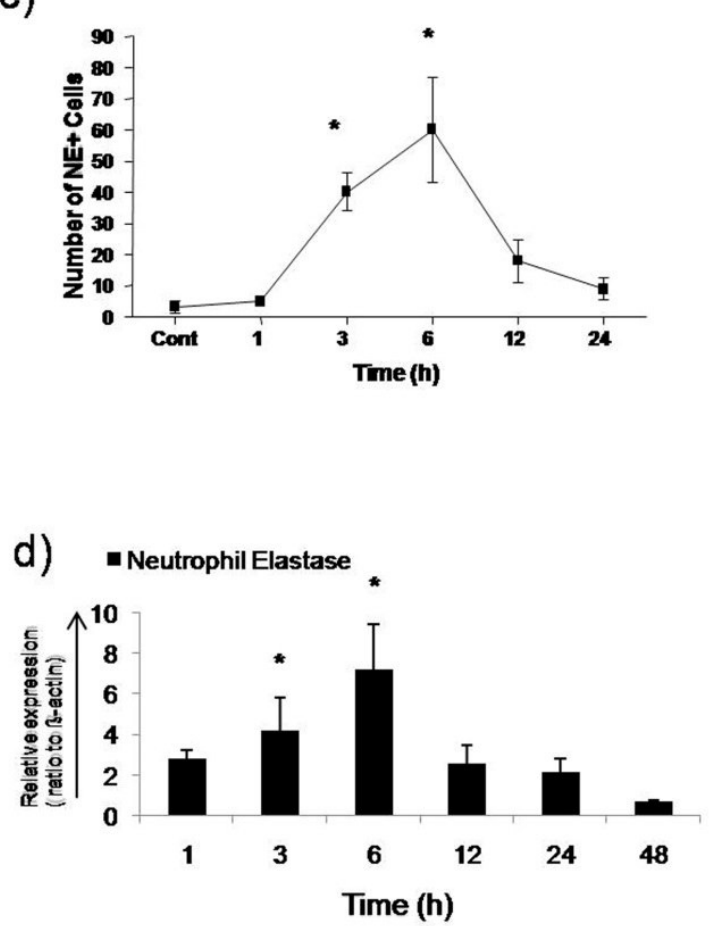

Figure 6: Indirect immunodetection of neutrophil elastase (NE) in sections of (a) sham-irradiated control rat liver and (b) rat liver 6 hours after irradiation (the arrows indicate the $\mathrm{NE}^{+}$granulocytes in the portal field) (original magnifications $\times 200$; the scale bars represent $100 \mu \mathrm{m}$ ). (c) $\mathrm{NE}^{+}$cells were counted in the portal fields of view $(\mathrm{N}=10)$ at different time points following rat liver irradiation. (d) Changes in the levels of hepatic NE messenger ribonucleic acid (mRNA) expression at the different time points following rat liver irradiation, which were determined by utilising the reverse transcriptase-polymerase chain reaction. The upregulation of NE mRNA was detected at 1 hour after irradiation and it peaked at 6 hours after irradiation, after which it declined $(\mathrm{N}=3)$. Abbreviations: NE, neutrophil elastase; cont, control.

\subsubsection{Identification of the recruited neutrophils and macrophages using an immunofluorescence double-staining protocol}

An immunofluorescence double-staining protocol was used to examine the inflammatory cell population that entered the liver after gamma-irradiation using cluster of differentiation (CD)11b/c and ED1 antibodies.

$\mathrm{CD} 11 \mathrm{~b} / \mathrm{c}$ is a marker for neutrophils and macrophages. Monocytes and inflammatory macrophages express the antigen ED1, while some resident macrophages are ED2 ${ }^{+}$, monocytes and dendritic cells are ED2 (Dijkstra et al. 1985). 
$\mathrm{CD} 11 \mathrm{~b} / \mathrm{c}^{+} \mathrm{ED} 1^{-}$cells showed a rapid and early accumulation around the portal vessels 3-6 hours after the irradiation in the rat liver. The CD11b/c ${ }^{+}$cells began to accumulate 1 hour after the irradiation of the rat liver and they only accumulated around the portal vessels (Fig. 7). The numbers of CD11b/c $\mathrm{c}^{+}$cells in the portal area had declined at 12 hours and 24 hours after the irradiation of the rat liver. $\mathrm{CD} 11 \mathrm{~b} / \mathrm{c}^{+} \mathrm{ED} 1^{+}$cells were present in the parenchyma and these corresponded to the Kupffer cells. In the parenchyma no increases in the number of $\mathrm{CD} 11 \mathrm{~b} / \mathrm{c}^{+} \mathrm{ED} 1^{+}$cells occurred after irradiation as well as in the central area compared to numbers of $\mathrm{CD} 1 \mathrm{~b} / \mathrm{c}^{+} \mathrm{ED} 1^{+}$in the livers of the control animals (Fig. 7).

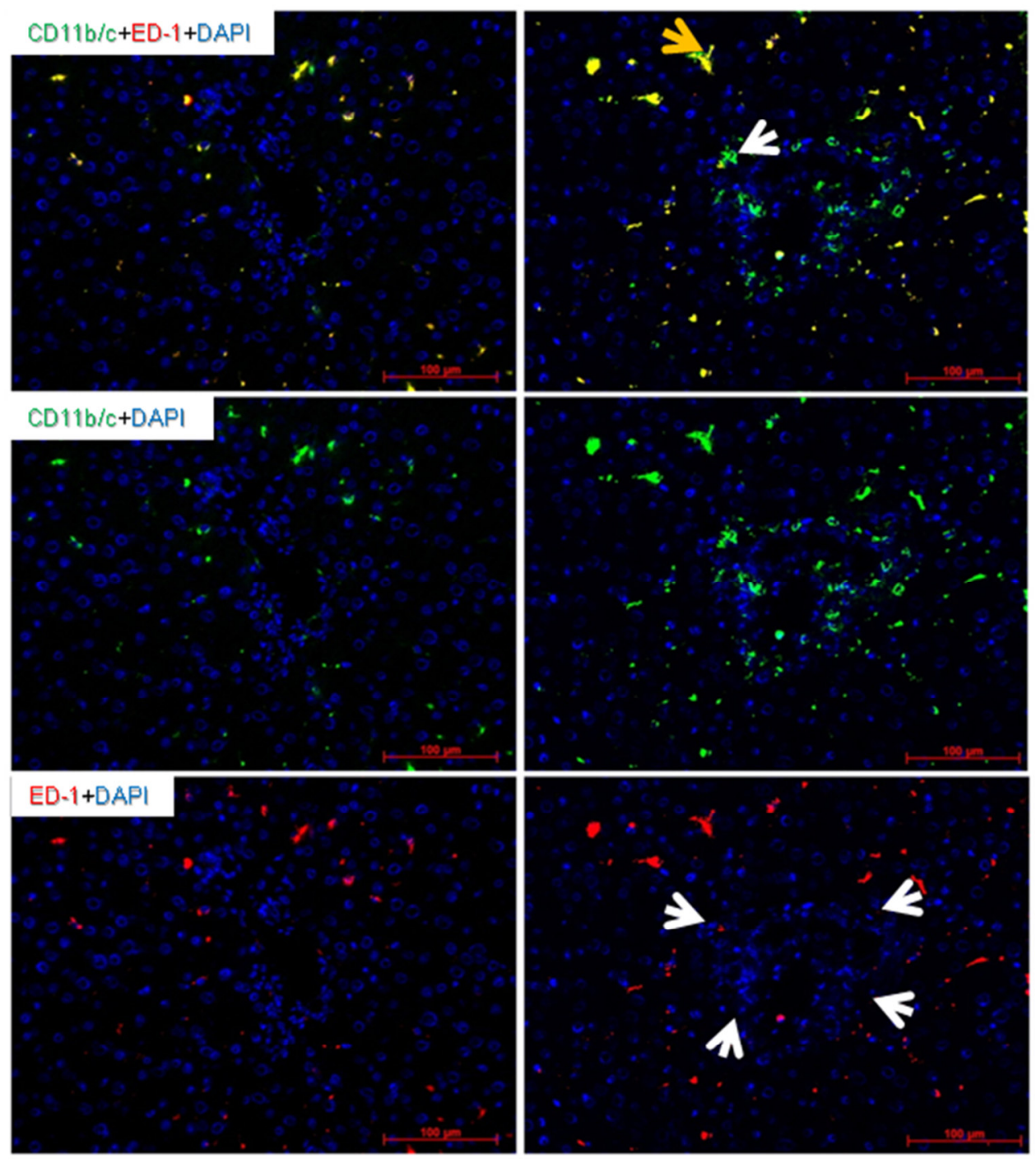

Figure 7: Double immunofluorescence staining of rat hepatic slices using monoclonal antibodies against ED1 (red) and rat cluster of differentiation (CD) $11 \mathrm{~b} / \mathrm{c}$ (green) after irradiation. The images on the left correspond to the livers from the sham-irradiated animals and the images on the right correspond to the livers from the irradiated animals at 6 hours after irradiation. The yellow arrow indicates the $\mathrm{ED} 1^{+} \mathrm{CD} 11 \mathrm{~b} / \mathrm{c}^{+}$resident macrophages, the white arrow indicates the recruited $\mathrm{CD} 11 \mathrm{~b} / \mathrm{c}^{+} \mathrm{ED} 1^{-}$neutrophils. The images at the top of the panel show double immunofluorescence staining for CD11b/c and ED1, the images in the middle of the panel 
show $\mathrm{CD} 11 \mathrm{~b} / \mathrm{c}$ staining and the images at the bottom of the panel show ED1 staining and the arrowheads indicate the portal area. The results represent the mean values from three rats and 6-slides for each time-point (original magnifications $\times 200$; scale bars represent $100 \mu \mathrm{m}$ ). Abbreviations: $\mathrm{CD}$, cluster of differentiation; DAPI, 4',6-diamidino-2-phenylindole.

No changes were evident with respect to the numbers of $\mathrm{ED}^{+}$and $\mathrm{ED}^{+}$tissue phagocytes (Fig. 8a-d). Confirmation of these results was achieved by counting the cells around the portal area (Fig. 8e \& 8f).
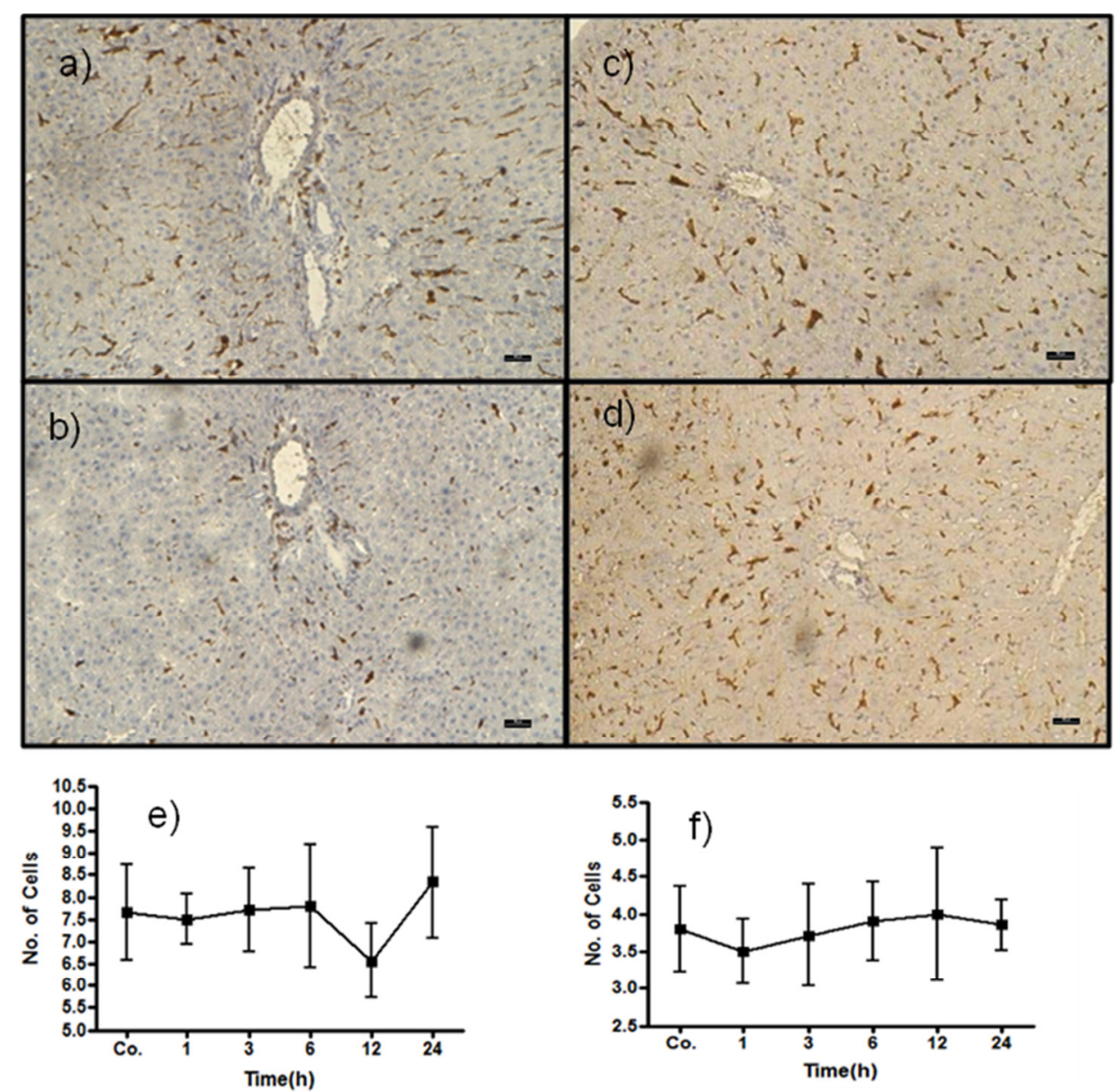

Figure 8: $\mathrm{ED} 1^{+}$and $\mathrm{ED} 2^{+}$cells in rat livers after irradiation. (a) $\mathrm{ED} 1^{+}$cells in a sham-irradiated liver, (b) $\mathrm{ED} 1^{+}$ cells in a liver 6 hours after irradiation, (c) ED2 ${ }^{+}$cells in a sham-irradiated liver and (d) ED2 ${ }^{+}$cells in a liver 6 hours after irradiation (original magnifications $\times 100$; the scale bars represent $100 \mu \mathrm{m}$ ). (e) ED1 ${ }^{+}$cells and (f) $\mathrm{ED} 2^{+}$cells were counted in the areas around the portal vessels at the different time points after rat liver irradiation. The results represent the mean values from three animals and six slides per time point. Abbreviation: Co, control.

Using antibodies to different surface markers enabled the detection of neutrophilic infiltrations that were only present around the portal vessel. Furthermore, no recruitment of activated or resident macrophages was detected after gamma-irradiation. 


\subsubsection{Relationship between neutrophils and liver myofibroblasts}

After observing that the macrophage numbers did not increase and that the neutrophils were located in the vicinities of the portal vessels only, an analysis of the liver myofibroblasts (LMFs) within the portal area was required. Double immunofluorescence staining using antibodies against $\mathrm{CD} 1 \mathrm{~b} / \mathrm{c}$ and smooth muscle actin (SMA), which is a myofibroblast marker, showed that the $\mathrm{SMA}^{+}$LMFs were surrounded by neutrophils (Fig. 9), thereby emphasising the strong association between the neutrophils and the vessels of the portal area.

a)
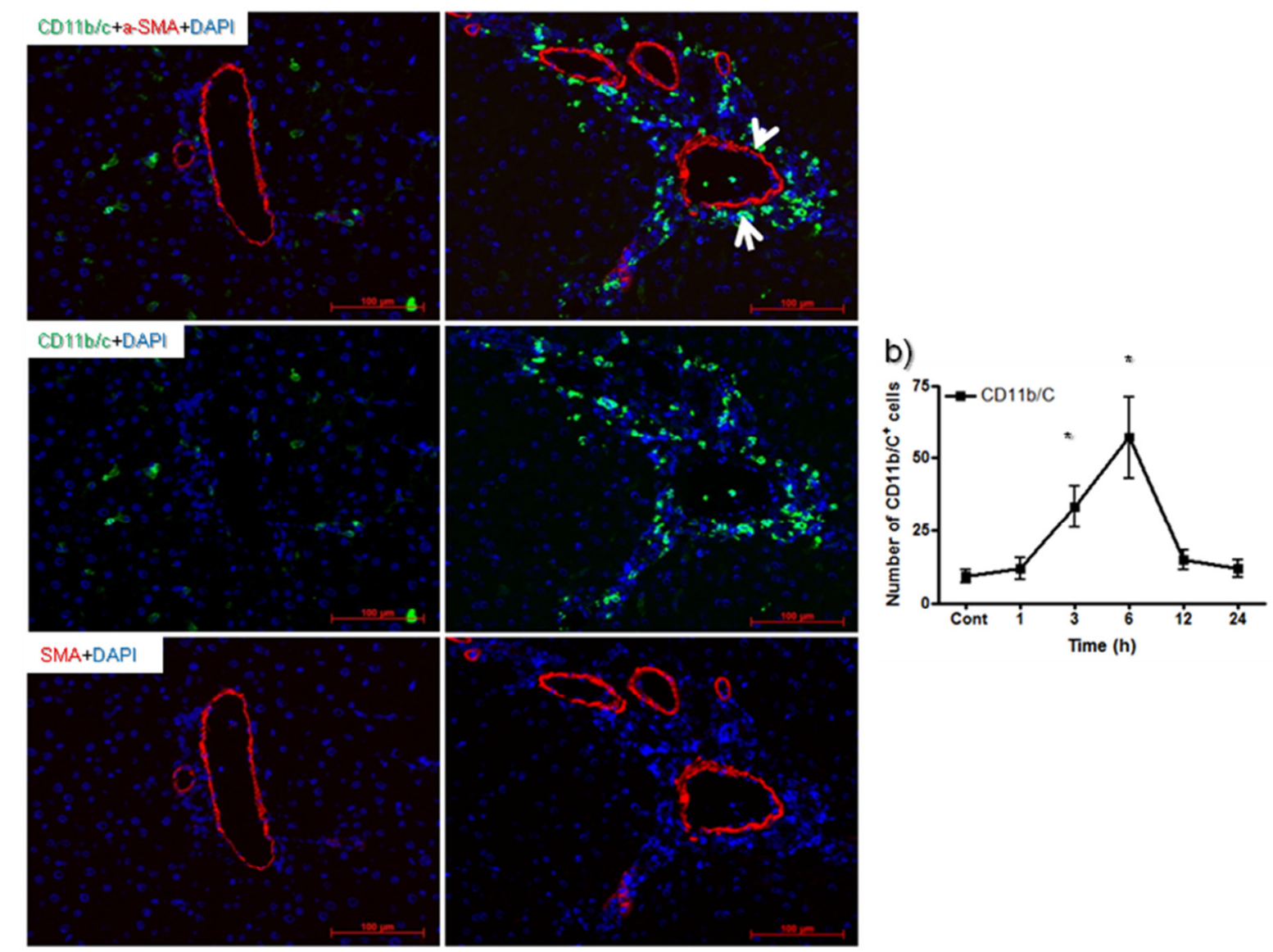

Figure 9: Double immunofluorescence staining of rat hepatic sections with monoclonal antibodies against smooth muscle actin (SMA) (red) and cluster of differentiation (CD)11b/c (green) (original magnifications $\times$ 200; the scale bars represent $100 \mu \mathrm{m}$ ). (a) The images on the left correspond to the livers from the shamirradiated animals and the images on the right correspond to the livers from the irradiated animals at 6 hours after irradiation. The white arrows indicate the recruited neutrophils that were present around the portal vessels. The images at the top of the panel show double immunofluorescence staining for CD11b/c and SMA, the images in the middle of the panel show CD11b/c staining and the images at the bottom of the panel show SMA staining. (b) The $\mathrm{CD} 11 \mathrm{~b} / \mathrm{c}^{+}$cells were counted in the portal fields of view $(\mathrm{N}=10)$ at different time points after rat liver irradiation $(\mathrm{N}=3)$. Abbreviations: $\mathrm{CD}$, cluster of differentiation; DAPI, 4',6-diamidino-2-phenylindole; SMA, smooth muscle actin. 
Thy-1 is another marker of periportal fibroblasts (Dudas et al. 2009), and double immunofluorescence staining using antibodies against CD11b/c and Thy-1 further supported the results obtained from the double immunofluorescence staining of the liver tissues for SMA and CD11b/c. Thy $-1^{+}$cells were present in the portal vessels' walls and within the portal tracts next to the walls of the portal vein and within those adjacent to the hepatic artery. The Thy $-1^{+}$cells were in close contact with the newly recruited CD $11 \mathrm{~b} / \mathrm{c}^{+}$neutrophils (Fig. $10)$.

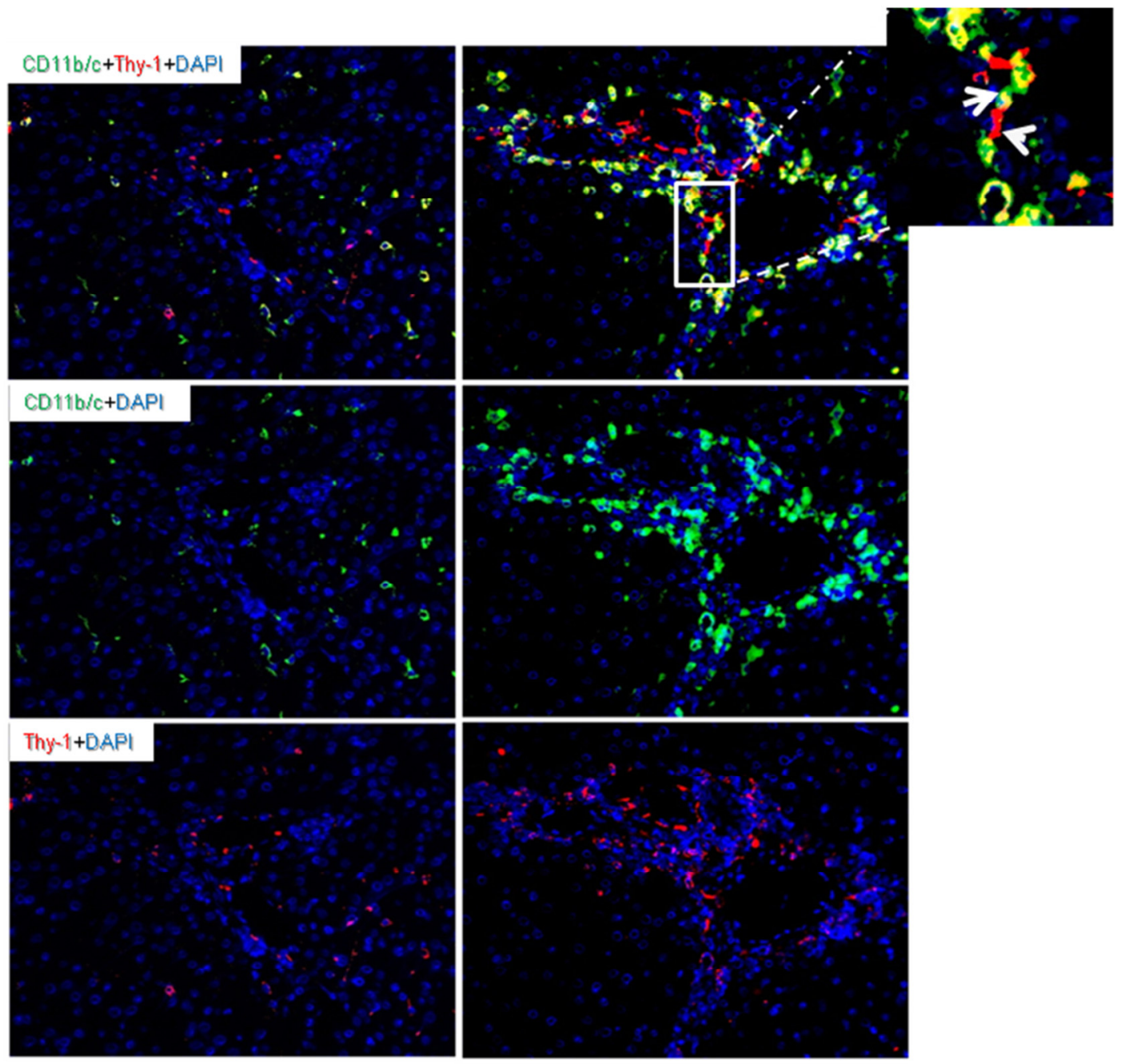

Figure 10: Double immunofluorescence staining of rat liver sections with monoclonal antibodies against Thy-1 (red) and cluster of differentiation (CD)11b/c (green). The images on the left correspond to the livers from the sham-irradiated animals and the images on the right correspond to the livers from the irradiated animals at 6 hours after irradiation. The images at the top of the panel show double immunofluorescence staining for CD11b/c and Thy-1, the images in the middle of the panel show CD11b/c staining and the images at the bottom of the panel show Thy-1 staining. The upper right image shows the periportal location of the neutrophils between and around the liver myofibroblasts (LMFs) $\left(\right.$ Thy $\left.-1^{+}\right)$. The white arrow indicates the recruited neutrophils that were present around the portal vessels and the white arrow head indicates the LMFs that appear to be closely 
associated with the neutrophils (original magnifications $\times 200$; the scale bars represent $100 \mu \mathrm{m}$ ). The results represent the mean values from three animals and six slides per time point. Abbreviations: $\mathrm{CD}$, cluster of differentiation; DAPI, 4',6-diamidino-2-phenylindole.

Taken together, these immunohistological data demonstrate that the neutrophils was the only type of leukocytes recruited into the portal area of the rat liver after a single dose of gamma-irradiation. Furthermore, the recruited neutrophils appeared to have direct contact with the LMFs in the portal area.

\subsection{Immunohistochemical detection of monocyte chemoattractant protein-1/CCL2 in the irradiated rat liver}

Using an antibody against MCP-1/CCL2, the presence of MCP-1/CCL2 was detected in and adjacent to vicinities of the portal vessel walls within the rat liver, and the level of expression peaked 3 hours after the liver had been irradiated (Fig. 11). The expression of MCP-1/CCL2 declined progressively thereafter, but it remained detectable for up to 24 hours after irradiation (Fig. 11a-c). The increase in the number of MCP-1/CCL2 ${ }^{+}$cells was visible in the rat liver at 1 hour and it peaked at 3 hours $(12.1 \pm 1.7$ cells) after irradiation (Fig. 11d). The positive staining for MCP-1/CCL2 was noticed in the periportal area where the recruited neutrophils were present (Fig. 11). Furthermore, MCP-1/CCL2 positivity was revealed mainly in the portal vessels, and few positive cells were observed around the portal vessels.

\subsection{Microarray gene expression analysis}

Microarray technology enables changes in the expression patterns of thousands of genes within cells or tissues to be monitored simultaneously. In the current study, rat liver tissues were investigated after 6 hours of gamma-irradiation (irradiated group), compared with sham-irradiated control (control group) to determine changes in the gene expression patterns using a gene array from Affymetrix, Inc. This time point was selected for the gene expression analysis because the highest number of neutrophils was observed at this time point following irradiation. Three animals were selected from each of the control and irradiated groups. The array was designed to profile the expression of 20,000 genes known to be associated with the liver system. The quality of the raw data from the microarray was 


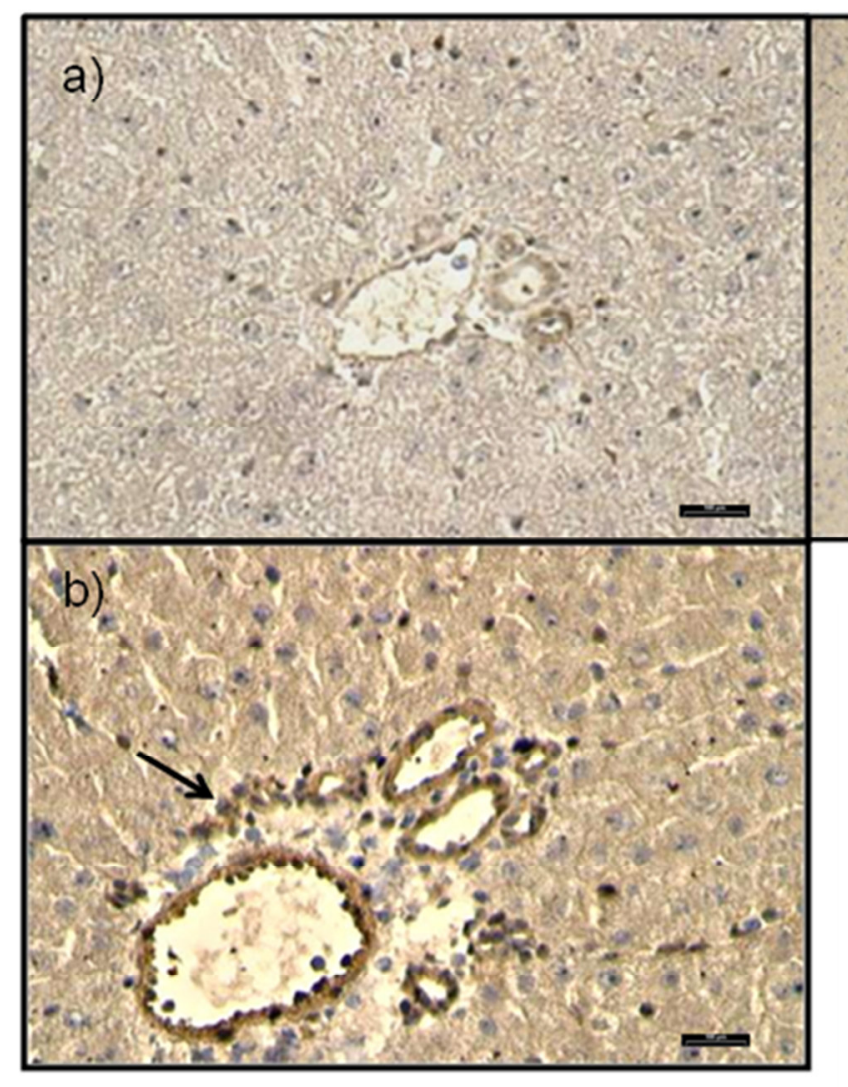

Portal area c)

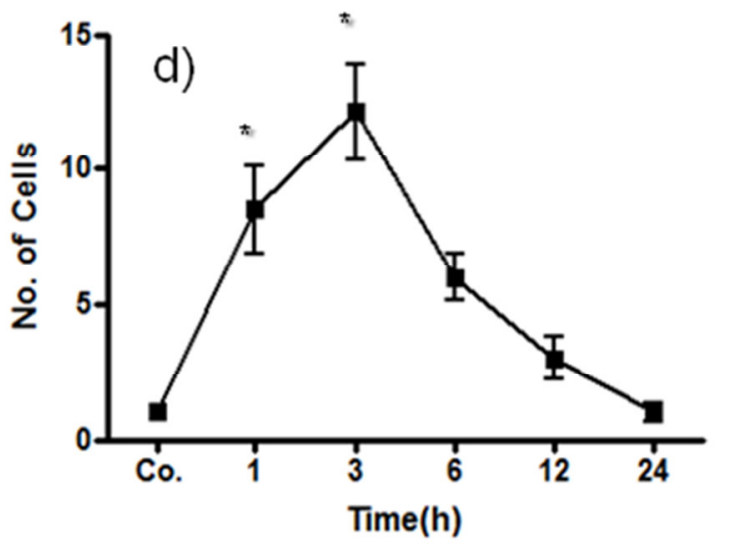

Figure 11: Immunodetection of monocyte chemoattractant protein (MCP)-1/CCL2 using a goat polyclonal antiserum against MCP-1/CCL2 followed by peroxidase staining in (a) sham-irradiated control rat liver sections and (b) rat liver sections 3 hours after irradiation. Compared with the livers from the sham-irradiated control animals, an increase intensity of MCP-1/CCL2 was observed within the walls of the portal vessels and in the cells accumulating around the vessels (black arrow) that peaked at 3 hours after irradiation (original magnifications $\times 200$; the scale bars represent $100 \mu \mathrm{m}$ ). (c) Negative control staining comprised the use of the secondary antibody and peroxidase staining (original magnification $\times 100$; the scale bar represents $100 \mu \mathrm{m}$ ). $(\mathrm{d}$ ) Numbers of MCP-1/CCL2 ${ }^{+}$cells in and around the portal field $(\mathrm{N}=10)$. The results represent mean values from three animals and six slides per time point. Abbreviation: Co, control.

controlled and the data were analysed using bioinformatics tools. Then, the data were further aligned and a spreadsheet was created. This spreadsheet comprised the gene symbols and the gene expression data were expressed as fold-changes. Based on the information obtained using the bioinformatics tools, hundreds of genes were found to be differentially regulated after irradiation compared with the sham-irradiated group.

The $P$ value was adjusted and the false discovery rate (FDR) was controlled. The FDR corrects for multiple comparisons and it is used to test multiple hypotheses while assuring few 
false-positive results. For each gene, the null hypothesis was set and the candidate genes were selected according to FDR criteria of fold changes of $<0.05$ and $\geq 1.7$. Using these criteria, the irradiated group had only 28 genes that were either upregulated or downregulated compared with the non-irradiated group, which are shown in a heat map. To obtain a higher number of differentially expressed genes, the criteria were re-evaluated and the FDR measures were changed to fold changes of $<0.065$ and $\geq 1.7$. Using these criteria, the rat livers in the irradiated group contained 55 genes that were differentially regulated in comparison to control group, the mRNA-level of 31 genes had increased and the expression of 24 of these genes had declined. The genes that were differentially regulated after rat liver irradiation are shown in tables 3 and 4 in which the arrows indicate the increases or decreases.

Compared with the control livers, Abcblb was the most upregulated gene (51.86-fold change), followed by Mybl1 (13.15-fold change) and CXCL1 (8.86-fold change). These genes are known to participate in regulating the immune system. Furthermore, the roles of Ccng1 and Cdknla in the cell cycle are well established.

Table 3: Upregulated ( $($ ) genes in the irradiated rat livers compared with the control livers.

\begin{tabular}{|c|c|c|c|c|}
\hline $\begin{array}{l}\text { Accession } \\
\text { number }\end{array}$ & $\begin{array}{l}\text { Increase in } \\
\text { expression } \\
\text { (fold change) }\end{array}$ & $\begin{array}{l}\text { Control vs } \\
\text { irradiated } \\
\text { liver }\end{array}$ & Gene symbol & Gene description \\
\hline NM_012623 & 51.86 & $\uparrow$ & Abcb1b & $\begin{array}{l}\text { ATP-binding cassette sub-family B } \\
\text { (MDR/TAP) member 1B }\end{array}$ \\
\hline NM_001106632 & 13.15 & $\uparrow$ & $\begin{array}{l}\text { Mybl1_predicte } \\
\text { d }\end{array}$ & $\begin{array}{l}\text { myeloblastosis oncogene-like } 1 \\
\text { (predicted) }\end{array}$ \\
\hline NM_030845 & 8.86 & 4 & Cxcl1 & chemokine (C-X-C motif) ligand 1 \\
\hline NM_012923 & 8.15 & 4 & Cong1 & cyclin G1 \\
\hline NM_080782 & 7.84 & $\uparrow$ & Cdkn1a & cyclin-dependent kinase inhibitor $1 \mathrm{~A}$ \\
\hline NM_170668 & 5.54 & $\uparrow$ & Slc13a5 & $\begin{array}{l}\text { solute carrier family } 13 \text { (sodium- } \\
\text { dependent citrate transporter) member } 5\end{array}$ \\
\hline NM_012551 & 5.39 & 4 & Egr1 & early growth response 1 \\
\hline NM_001106536 & 5.34 & 4 & $\begin{array}{l}\text { Mybl2_predicte } \\
\text { d }\end{array}$ & $\begin{array}{l}\text { myeloblastosis oncogene-like } 2 \\
\text { (predicted) }\end{array}$ \\
\hline NM_001109203 & 5.19 & $\mathbf{4}$ & Prrg4 & $\begin{array}{l}\text { proline rich Gla (G-carboxyglutamic acid) } 4 \\
\text { (transmembrane) }\end{array}$ \\
\hline NM_001012206 & 5.02 & 4 & Phlda3 & $\begin{array}{l}\text { pleckstrin homology-like domain family A } \\
\text { member } 3\end{array}$ \\
\hline
\end{tabular}




\begin{tabular}{|c|c|c|c|c|}
\hline NM_001108099 & 4.63 & 4 & $\begin{array}{l}\text { Mdm2_predicte } \\
\text { d }\end{array}$ & $\begin{array}{l}\text { transformed mouse } 3 T 3 \text { cell double } \\
\text { minute } 2 \text { homologue (mouse) (predicted) }\end{array}$ \\
\hline $\begin{array}{l}\text { ENSRNOT0000001 } \\
7429\end{array}$ & 3.79 & $\uparrow$ & $\begin{array}{l}\text { Eda2r_predicte } \\
\text { d }\end{array}$ & $\begin{array}{l}\text { ectodysplasin A2 isoform receptor } \\
\text { (predicted) }\end{array}$ \\
\hline NM_001077589 & 3.75 & $\uparrow$ & Rgs16 & regulator of G-protein signalling 16 \\
\hline NM_001010965 & 3.56 & 4 & Rage & renal tumour antigen \\
\hline NM_031784 & 3.49 & 4 & Pias3 & protein inhibitor of activated STAT 3 \\
\hline NM_022542 & 3.36 & 4 & Rhob & ras homologue gene family member $B$ \\
\hline NM_031588 & 3.30 & 4 & Nrg1 & neuregulin 1 \\
\hline $\begin{array}{l}\text { ENSRNOT0000000 } \\
3339\end{array}$ & 3.07 & 4 & Trim7 & tripartite motif-containing 7 \\
\hline $\begin{array}{l}\text { ENSRNOT0000002 } \\
4369\end{array}$ & 3.00 & $\uparrow$ & Adam8 & $\begin{array}{l}\text { a disintegrin and metallopeptidase } \\
\text { domain } 8\end{array}$ \\
\hline $\begin{array}{l}\text { ENSRNOT0000000 } \\
8981\end{array}$ & 2.81 & 4 & Lama5 & laminin alpha 5 \\
\hline $\begin{array}{l}\text { ENSRNOT0000003 } \\
0102\end{array}$ & 2.76 & 4 & Il17rd & interleukin 17 receptor $D$ \\
\hline NM_012674 & 2.61 & 4 & Spink1 & serine protease inhibitor Kazal type 1 \\
\hline NM_022242 & 2.33 & 4 & Niban & niban protein \\
\hline NM_001044304 & 2.25 & 4 & Eid3 & $\begin{array}{l}\text { EP300 interacting inhibitor of } \\
\text { differentiation } 3\end{array}$ \\
\hline $\begin{array}{l}\text { ENSRNOT0000002 } \\
9629\end{array}$ & 2.13 & $\uparrow$ & LOC683565 & hypothetical protein LOC683565 \\
\hline $\begin{array}{l}\text { ENSRNOT0000002 } \\
4858\end{array}$ & 2.1 & 4 & $\begin{array}{l}\text { Zfp688_predicte } \\
\text { d }\end{array}$ & zinc finger protein 688 (predicted) \\
\hline $\begin{array}{l}\text { ENSRNOT0000002 } \\
5716\end{array}$ & 2.08 & 4 & LOC690769 & similar to zinc ring finger protein 1 \\
\hline NM_012861 & 2 & $\uparrow$ & Mgmt & $\begin{array}{l}\text { O-6-methylguanine-DNA } \\
\text { methyltransferase }\end{array}$ \\
\hline NM_001024900 & 1.97 & 4 & Tmem55a & transmembrane protein 55A \\
\hline $\begin{array}{l}\text { ENSRNOT0000004 } \\
2973\end{array}$ & 1.94 & 4 & Tnfaip2 & $\begin{array}{l}\text { tumour necrosis factor alpha-induced } \\
\text { protein } 2\end{array}$ \\
\hline $\begin{array}{l}\text { ENSRNOT0000004 } \\
8849\end{array}$ & 1.84 & $\uparrow$ & --- & --- \\
\hline
\end{tabular}


Among the downregulated genes, Ccrn4lb was the most downregulated (10.73-fold change), followed by Scrn1 (6.36-fold change) and RGD1565709_predicted (similar to ovostatin-2) (5.31-fold change) in the irradiated group compared with the control group. Ccrn4lb plays a role in metabolic functions, and Scrn1 and ovostatin-2 are thought to be involved in carcinogenesis. The details of the downregulated genes are summarised in table 4 .

Table 4: Downregulated $(\downarrow)$ genes in the irradiated rat livers compared with the control livers.

\begin{tabular}{|c|c|c|c|c|}
\hline $\begin{array}{l}\text { Accession } \\
\text { number }\end{array}$ & $\begin{array}{l}\text { Decrease in } \\
\text { expression } \\
\text { (fold change) }\end{array}$ & $\begin{array}{l}\text { Control vs } \\
\text { irradiated }\end{array}$ & Gene symbol & Gene description \\
\hline NM_138526 & 10.73 & $\downarrow$ & Ccrn4lb & $\begin{array}{l}\text { CCR4 carbon catabolite repression 4-like B } \\
\text { (Saccharomyces cerevisiae) }\end{array}$ \\
\hline NM_001025063 & 6.36 & $\downarrow$ & Scrn1 & secernin 1 \\
\hline $\begin{array}{l}\text { ENSRNOTO000000 } \\
9557\end{array}$ & 5.31 & $\downarrow$ & $\begin{array}{l}\text { RGD1565709_p } \\
\text { redicted }\end{array}$ & similar to ovostatin-2 (predicted) \\
\hline NM_024391 & 3.89 & $\downarrow$ & Hsd17b2 & hydroxysteroid (17-beta) dehydrogenase 2 \\
\hline NM_199113 & 3.35 & $\downarrow$ & Popdc2 & popeye domain containing 2 \\
\hline NM_057107 & 3.26 & $\downarrow$ & Acsl3 & $\begin{array}{l}\text { acyl-CoA synthetase long-chain family } \\
\text { member } 3\end{array}$ \\
\hline $\begin{array}{l}\text { ENSRNOT0000001 } \\
4054\end{array}$ & 3.17 & $\downarrow$ & Atxn7|1 & ataxin 7-like 1 \\
\hline NM_022249 & 2.94 & $\downarrow$ & Khdrbs3 & $\begin{array}{l}\text { KH domain containing RNA binding signal } \\
\text { transduction associated } 3\end{array}$ \\
\hline $\begin{array}{l}\text { ENSRNOT0000004 } \\
0778\end{array}$ & 2.93 & $\downarrow$ & LOC301455 & $\begin{array}{l}\text { similar to amyotrophic lateral sclerosis } 2 \\
\text { (juvenile) chromosome region candidate } \\
19 \text { isoform b }\end{array}$ \\
\hline NM_001108492 & 2.89 & $\downarrow$ & Syt12_predicted & synaptotagmin-like 2 (predicted) \\
\hline NM_012689 & 2.84 & $\downarrow$ & Esr1 & oestrogen receptor 1 (alpha) \\
\hline $\begin{array}{l}\text { ENSRNOT0000002 } \\
7493\end{array}$ & 2.83 & $\downarrow$ & $\begin{array}{l}\text { RGD1310209_p } \\
\text { redicted }\end{array}$ & similar to KIAA1324 protein (predicted) \\
\hline NM_001007691 & 2.66 & $\downarrow$ & Prss23 & protease serine 23 \\
\hline NM_017127 & 2.60 & $\downarrow$ & Chka & choline kinase alpha \\
\hline NM_001105811 & 2.46 & $\downarrow$ & Rilp_predicted & $\begin{array}{l}\text { Rab interacting lysosomal protein } \\
\text { (predicted) }\end{array}$ \\
\hline NM_001108178 & 2.41 & $\downarrow$ & Pls1_predicted & plastin 1 (I isoform) (predicted) \\
\hline NM_001109606 & 2.38 & $\downarrow$ & Fbxo3 & F-box protein 3 \\
\hline NM_138912 & 2.29 & $\downarrow$ & Ppp1r3b & protein phosphatase 1 regulatory \\
\hline
\end{tabular}




\begin{tabular}{|l|l|c|l|l|}
\hline & & & & (inhibitor) subunit 3B \\
\hline NM_012868 & 2.17 & $\downarrow$ & Npr3 & natriuretic peptide receptor 3 \\
\hline $\begin{array}{l}\text { ENSRNOT0000001 } \\
8421\end{array}$ & 2.06 & $\downarrow$ & & $\begin{array}{l}\text { transducin-like enhancer of split 1 } \\
\text { homologue of Drosophila E(spl) } \\
\text { (predicted) }\end{array}$ \\
\hline NM_139258 & 2.01 & $\downarrow$ & Bmf & Bcl2 modifying factor \\
\hline NM_012842 & 1.97 & $\downarrow$ & Egf & epidermal growth factor \\
\hline ENSRNOT0000001 & & $\downarrow$ & RGD1560736_p & $\begin{array}{l}\text { similar to solute carrier family 9 } \\
\text { (sodium/hydrogen exchanger) isoform 9 } \\
\text { (predicted) }\end{array}$ \\
\hline 1358 & 1.82 & redicted & Insig1 & insulin induced gene 1 \\
\hline NM_022392 & 1.70 & $\downarrow$ & &
\end{tabular}

The microarray data were further analysed and heat map and principal component analysis (PCA) graphs were created.

\subsubsection{Heat map of differentially expressed genes}

Heat maps present data graphically as colours. The heat map presents the ztransformed $\log _{2}$ expression values for all of the significantly differentially expressed genes based on comparisons of the irradiated rats with the control rats using the FDR criteria of fold changes of $<0.05$ and $\geq 1$.7. A significantly differential expression of twenty-eight genes was detected post-irradiation in comparison to control group. Seventeen genes were induced and 11 genes were repressed in the livers of irradiated group compared with sham-irradiated control group. On the heat map, the upregulated and downregulated genes are red and blue, respectively (Fig. 12).

\subsubsection{Principal component analysis}

PCA provides the means by which high-dimensional data can be visualised within lower dimensional spaces. PCA is a multivariate data analysis method, and it was used to visualise the similarities and dissimilarities among the genome-wide expression profiles. Fig. 13 presents the PCA data for the normalised microarray dataset, which clearly shows that the samples from the irradiated group were more similar to each other than they were to the samples from the control group. Moreover, the differences between the irradiated and the control groups are evident. The first and second axes of the plot accounted for $21.27 \%$ and $20.84 \%$ of the data's overall variance, respectively (Fig. 13). 
Heat Map candidates IRR Vs Control

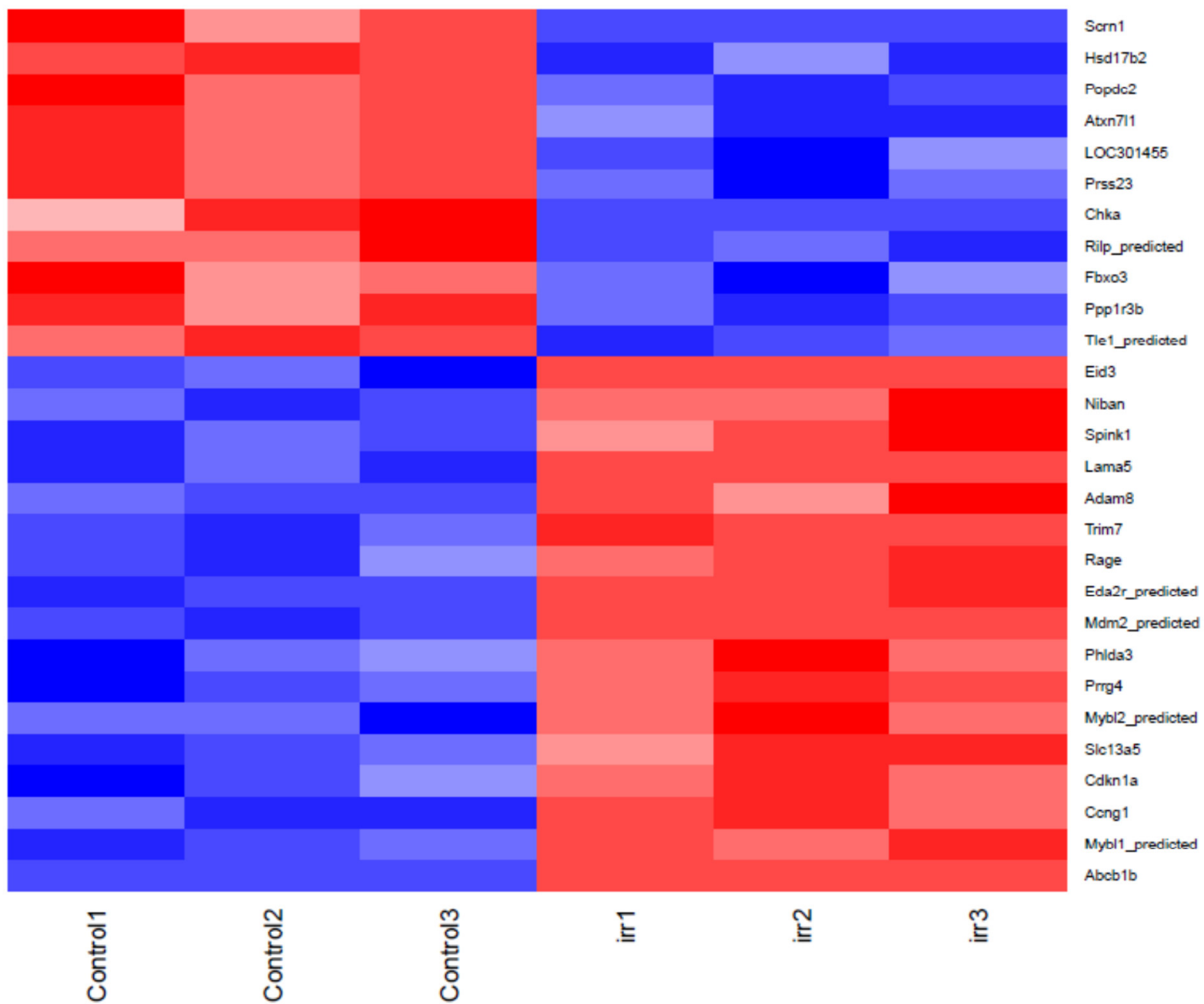

Figure 12: Heat map analysis of the microarray data. Gene-upregulation and -downregulation is shown in red and blue, respectively, in the corresponding sample columns. The liver samples from the control animals (C1, C2 and C3) were compared with those from the irradiated animals (Irr1, Irr2 and Irr3) 6 hours after irradiation. 


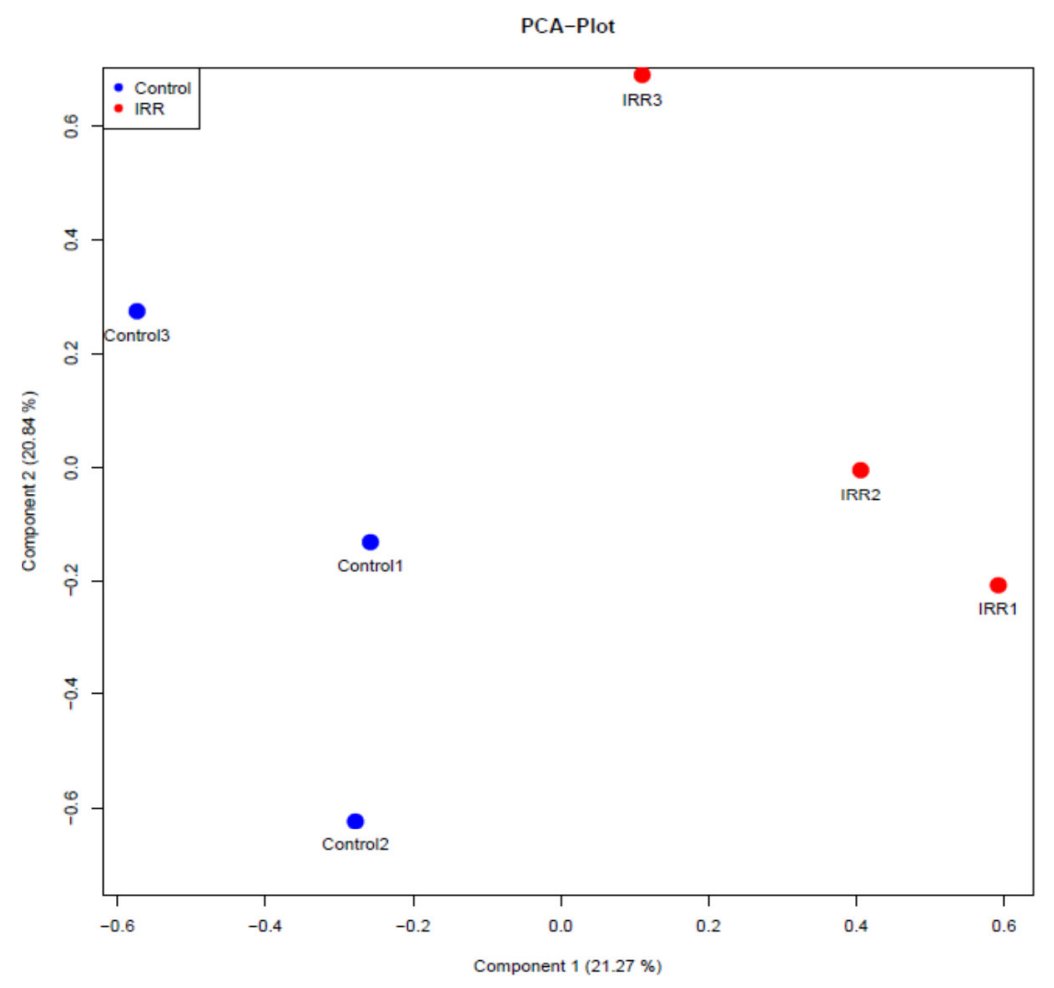

Figure 13: Principal component analysis (PCA) of the microarray data. The PCA represents the variability among the irradiated and the control samples. The control samples are presented in blue and the irradiated samples are presented in red. The liver samples from the control animals $(\mathrm{C} 1, \mathrm{C} 2$ and $\mathrm{C} 3)$ were compared with those from the irradiated animals (Irr1, Irr2 and Irr3) 6 hours after irradiation.

\subsection{Chemokine and chemokine-receptor gene expression in irradiated rat livers}

The microarray gene expression data demonstrated the detection of only a few differentially expressed genes in the irradiated livers compared with the control livers. Moreover, only one gene was detected that was associated with the cell transmigration process. Therefore, the RT-PCR was performed using gamma-irradiated and sham-irradiated control liver tissues to determine which genes might be associated with the higher neutrophil numbers in the periportal area. Compared with the control rat livers, the mRNA levels of CXCL1, CXCL2 and CXCL5 was upregulated 1 hour after irradiation and it peaked (CXCL1 [308.9 \pm 28.8-fold], CXCL2 [61.4 \pm 5.3 -fold], CXCL5 [52.5 \pm 11.47 -fold] at 3 hours after irradiation (Fig. 14a). The induction of the CXCL2 gene expression following liver irradiation was confirmed using the polymerase chain reaction (PCR), and a clear difference was observed compared with the sham-irradiated control rats (Fig. 14b). The peak in the level of expression of the CXCL1 gene at 3 hours after irradiation that was observed using the RTPCR was confirmed by Northern blot and Western blot analyses (Fig. 14c \& d). The levels of 
expression of the CXCL1, CXCL2 and CXCL5 genes declined progressively after 3 hours, but they remained higher than the levels of these genes within the livers from the control animals until 48 hours after rat liver irradiation. The number of granulocytes rose in the area around the portal vessel at the same time as the number of CXCR2 transcripts increased (24.4 \pm 5 .6-fold), which peaked at 3 hours after rat liver irradiation (Fig. 14a).

The upregulation of the expression of the MCP-1/CCL2 and MCP-3/CCL7 genes peaked 1 hour after rat liver irradiation and statistically significant increases in the levels of expression were apparent for up to 6 hours after irradiation. However, the CCR2 gene was not upregulated, and the expression of the CCR2 gene was downregulated at 12 hours and 48 hours after rat liver irradiation (Fig. 15). RNA analyses confirmed the induction of several of the chemokines, and, of these, the gene for MCP-1/CCL2 was the first to be induced after rat liver irradiation.

a) CXC-chemokines

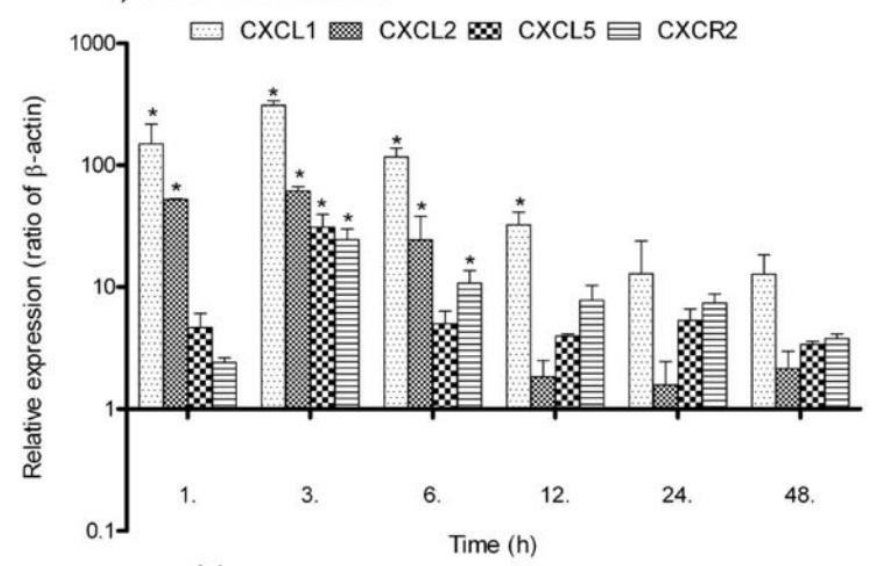

b)

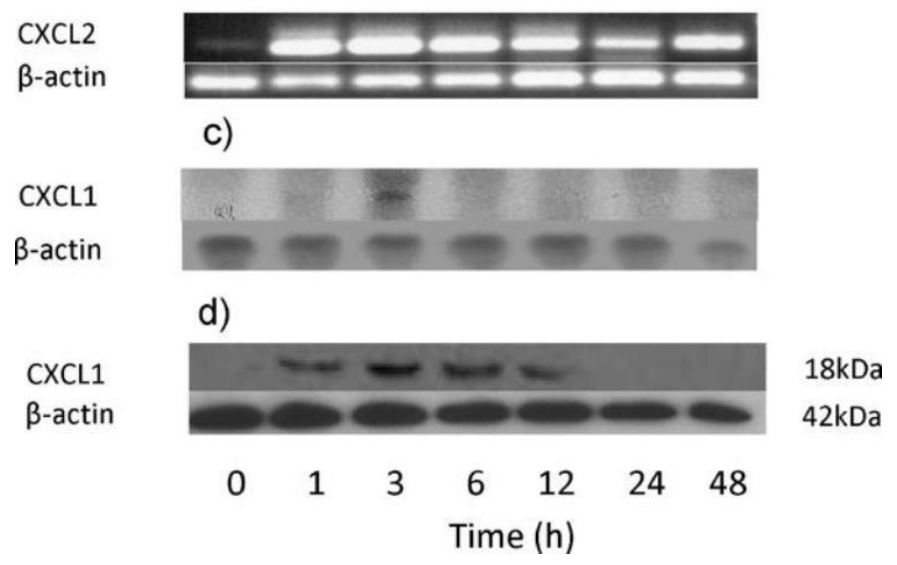

Figure 14: Alterations in messenger ribonucleic acid (mRNA) and protein-levels of the genes for CXC chemokines in rat livers. (a) Alterations in mRNA-level for the CXCL1, CXCL2 and CXCL5 chemokines and for CXCR2 in the irradiated livers at the different time points relative to the sham-irradiated control rats determined using the reverse transcriptase-polymerase chain reaction (RT-PCR). (b) Confirmation by agarose gel 
electrophoresis. Normalisation of the RT-PCR was achieved using the housekeeping genes, namely, beta-actin and ubiquitin, and the control value was set at one. (c) Northern blot analysis and (d) Western blot analysis using an antibody against CXCL1. Results represent the means \pm standard errors of the means from 3-experiments that were performed in duplicate and were compared with those from the sham-irradiated control rats at each time point ( $* P<0.05$, based on a one-way analysis of variance).

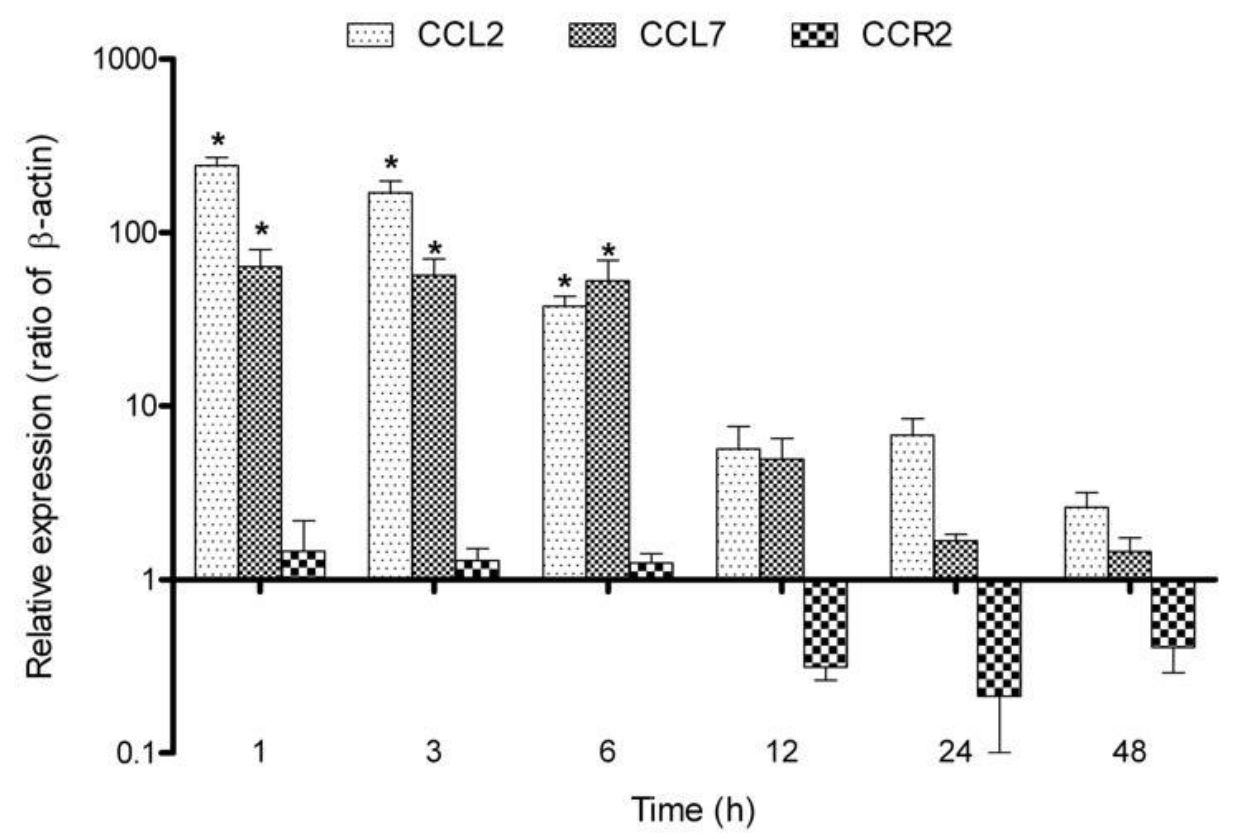

Figure 15: Changes in the levels of messenger ribonucleic acid expression for $\mathrm{CC}$ chemokines in the irradiated rat liver. The reverse transcriptase-polymerase chain reaction was normalised using two housekeeping genes, namely, beta-actin and ubiquitin $\mathrm{C}$. The control value was set at one. Results represent the means \pm standard errors of the means from 3-experiments that were performed in duplicate and compared with sham-irradiated control rats at each time point ( $* P<0.05$, based on a one-way analysis of variance).

\subsection{Changes in chemokine gene expression in microdissected irradiated rat liver sections}

To locate the periportal and parenchymal expression of the target chemokine genes, 10 $\mu \mathrm{m}$ liver sections from irradiated and sham-irradiated control animals were dissected using laser capture microdissection (LCM). Nine portal and nine parenchymal regions (three from each liver section) from each time point were microdissected and pooled. Then, following RNA extraction, the PCR was performed. Optimisation of the PCR protocol was achieved by varying the numbers of cycles and the annealing temperatures. Fifty cycles for the platelet/endothelial cell adhesion molecule (PECAM) and NE genes and 40 cycles for all of the other genes that were studied were standard for the PCR analysis. The expression of the 
A)

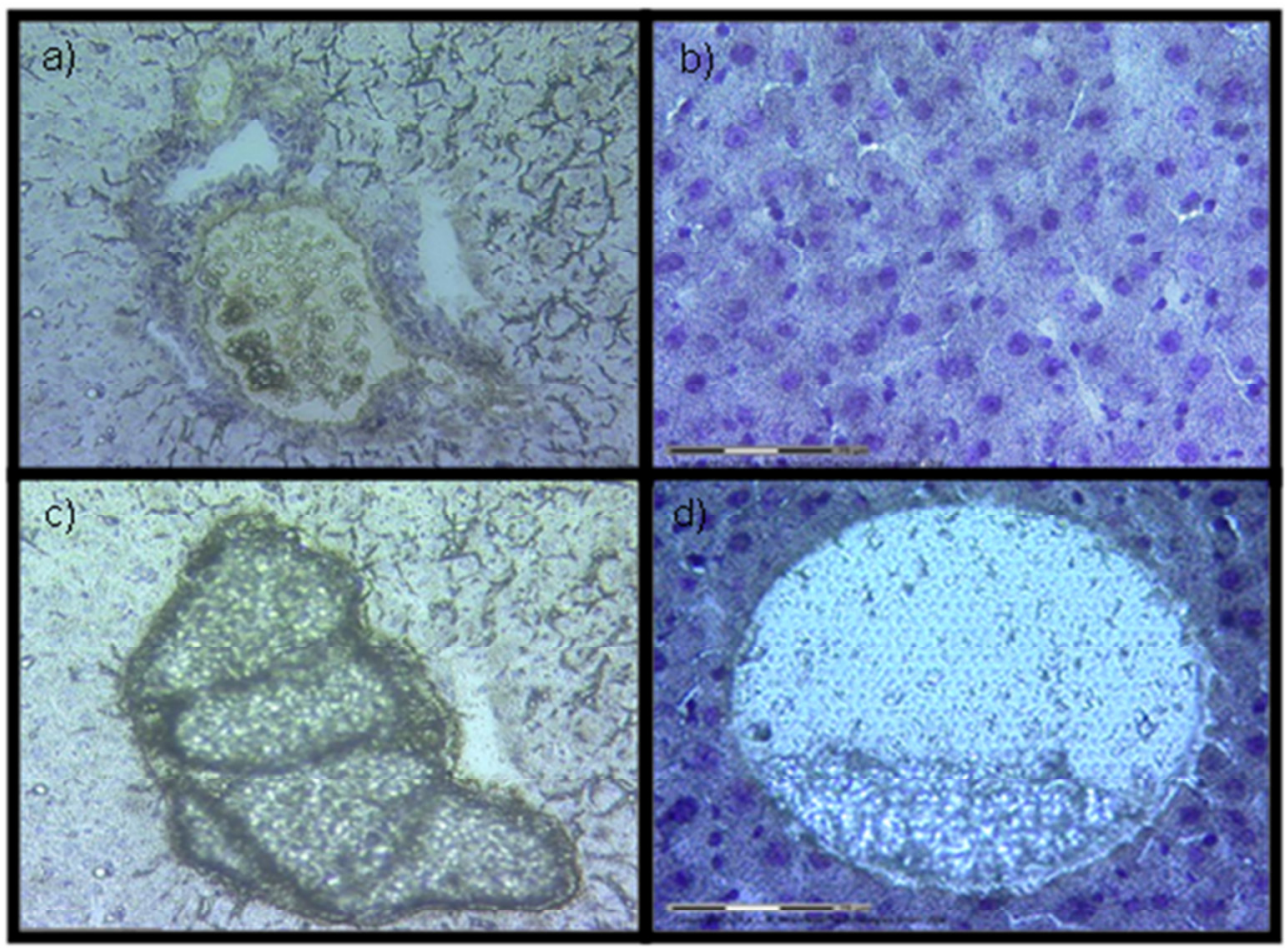

B)

Liver Portal area

Liver Parenchyma tissue

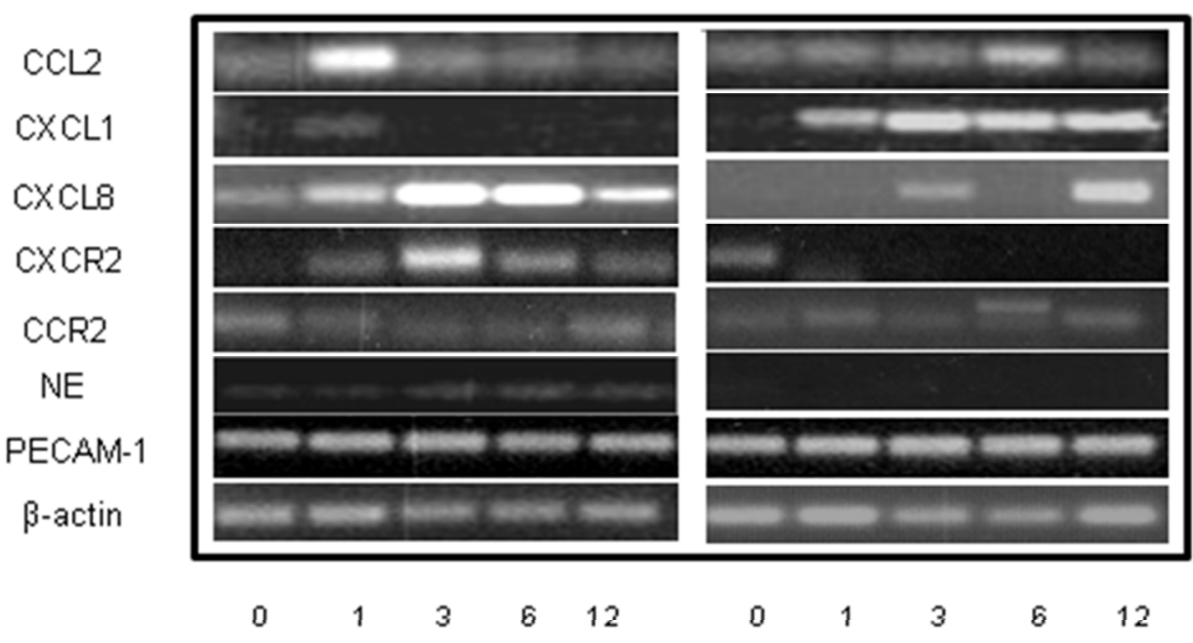

Time (h)

Figure 16: Laser-capture microdissection of rat liver sections after irradiation. A) Liver tissue sections were microdissected in the portal area (a) before cutting and (c) after cutting and in the parenchymal area (b) before cutting and (d) after cutting. B) Polymerase chain reaction (PCR) analysis of the total ribonucleic acid isolated from the microdissected liver tissues from the portal and parenchymal areas. Oligonucleotides specific for the different genes were used. The sizes of the PCR products were analysed using agarose (1\%) gel electrophoresis 
(ultraviolet light picture). Abbreviations: PECAM, platelet/endothelial cell adhesion molecule; NE, neutrophil elastase.

MCP-1/CCL2 and CXCL1 genes peaked at 1 hour after irradiation. The expression of the CXCL8 and CXCR2 genes was upregulated at 1 hour and it peaked in the microdissected cells from the portal area at 3 hours after rat liver irradiation. No changes were evident in relation to the expression of the MCP-1/CCL2 gene within the microdissected parenchymal tissue. The expression of the CXCL1 gene was intensely upregulated in the parenchyma, and it peaked at 3 hours after irradiation. The CXCR2 gene was only detectable in the microdissected parenchymal tissue from the sham-irradiated control animals. The expression of the NE gene increased over time in the microdissected portal area cells and it peaked at 6 hours after irradiation. NE gene expression was not detectable in the parenchymal cells. No changes were evident in relation to CCR2 gene expression in the portal or the parenchymal tissues at any time point. The PECAM-1 was taken as a positive control to enable comparisons of the expression of the chemokine genes. No changes in the expression of the PECAM-1 gene were apparent in either of the regions after irradiation (Fig. 16).

The analysis of the tissues using LCM demonstrated the induction of several chemokines within the periportal area after irradiation. MCP-1/CCL2 showed the earliest and most intense expression of the chemokines studied, which concurred with the observations in the whole liver tissue samples that had been irradiated. These data indicate the critical importance of MCP-1/CCL2 in liver inflammation induced by gamma-irradiation.

\subsection{Influence of an antibody against monocyte chemoattractant protein-1/CCL2 on hepatic gene expression after gamma-irradiation}

The immunohistological and PCR analyses indicated that MCP-1/CCL2 has an important role in neutrophil recruitment. Therefore, it was investigated whether irradiationinduced neutrophil infiltration could be hindered by neutralising MCP-1/CCL2 in the liver. Following the intraperitoneal administration of a monoclonal antibody against MCP-1/CCL2 to the rats shortly before irradiation (G3), there were no immunohistological changes observed in relation to neutrophil migration from 3 hours to 6 hours after irradiation compared with the animals that had been administered a single-dose of irradiation (G1). This was confirmed by counting the numbers of neutrophils in the portal area and measuring the levels of expression of the NE, CXCR2 and ED1 genes using the RT-PCR. The expression of the IFN-gamma gene was clearly downregulated in the livers of the rats that had been 
irradiated only (G1). The livers from the rats that had been administered the MCP-1/CCL2 antibody and had been irradiated (G3) showed an induction of the IFN-gamma gene (4.7 \pm 0.9 -fold) that was detected at 3 hours after irradiation. Similarly, a significant upregulation of IP-10/CXCL10 gene expression was noticed in the rats in G3 compared with those in G1 at 3 hours after irradiation (Fig. 17a, b).

Hence, blocking MCP-1/CCL2 with an antibody did not reduce the neutrophil numbers, but it induced changes in the expression of the hepatic genes. This finding indicates that blocking MCP-1/CCL2 was compensated by another chemokine, namely, IP10/CXCL10.
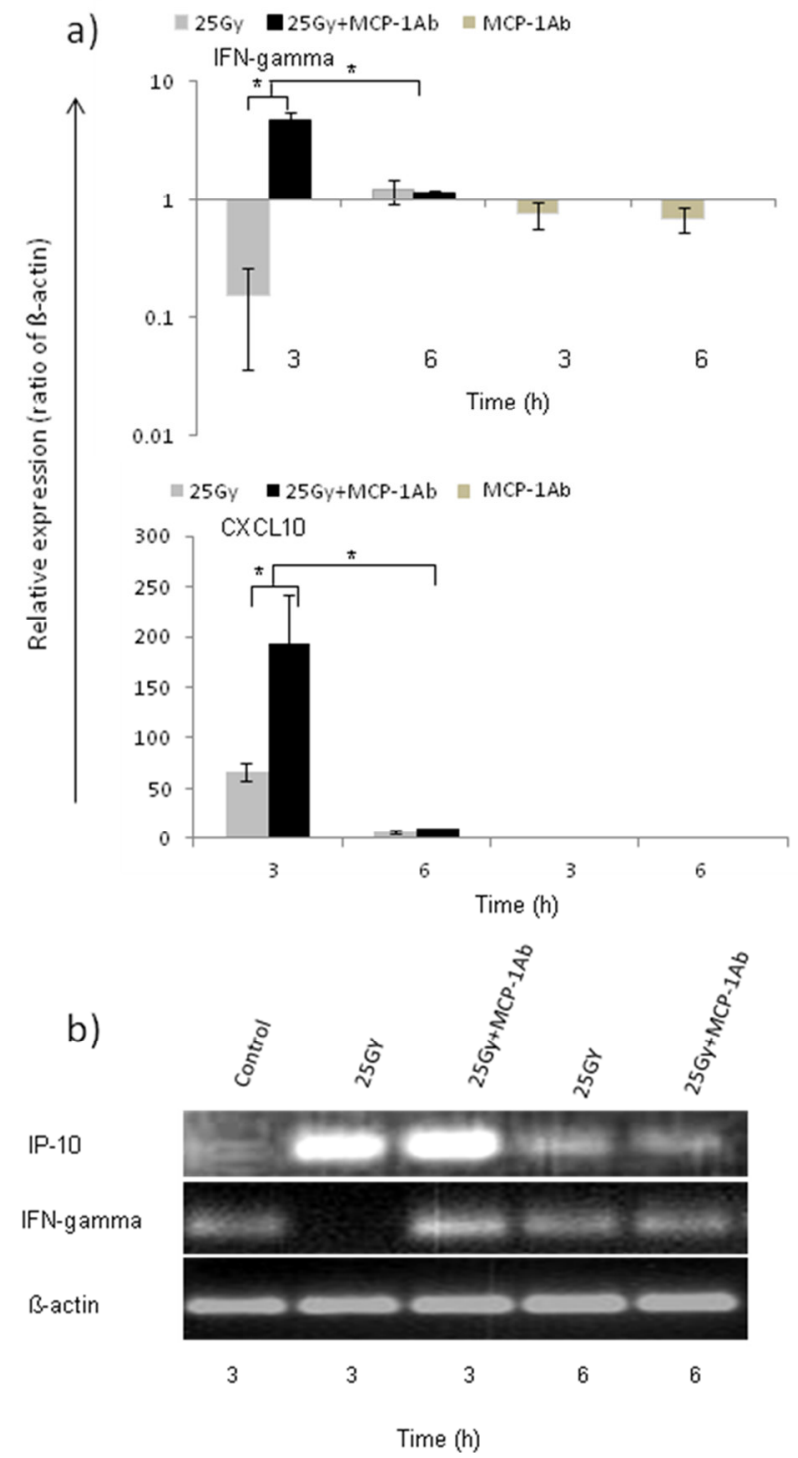

Figure 17: The effect of the intraperitoneal administration of a monoclonal antibody against monocyte chemoattractant protein-1/CCL2 on hepatic gene expression following gamma irradiation. (a) Changes in the levels of messenger ribonucleic acid (mRNA) expression for interferon (IFN)-gamma and for IFN-inducible 
protein CXCL10 in the rat hepatic tissue following irradiation compared to sham-irradiated control animals using the real-time polymerase chain reaction (RT-PCR). (b) The results obtained using RT-PCR analysis were reassured by running agarose-gel of the polymerase chain reaction products. The RT-PCR data is presented as fold changes. Normalisation of the RT-PCR was achieved using the housekeeping genes, namely, beta-actin and ubiquitin. The control value was set at one. The data represent the mean values from two experiments carried out in duplicate, and the means \pm the standard errors of the means were compared with those from sham-irradiated antibody-treated control animals at each time point $\left({ }^{*} P<0.05\right.$, based on a one-way analysis of variance).

Abbreviations: MCP, monocyte chemoattractant protein; IFN, interferon; IP-10, interferon-inducible protein

\subsection{Changes in chemokine and cytokine gene expression in the parenchymal and non- parenchymal cells of irradiated rat livers}

In an attempt to corroborate our in vivo data and to understand the interactions between the liver cells and the recruited neutrophils, changes in the levels of chemokines gene expression in parenchymal cells, that is, the hepatocytes, and non-parenchymal cells, that is, the LMFs, were investigated at 1, 3, 6, 12 and 24 hours after irradiation. They were compared with those in the cells from livers from sham-irradiated control animals. After preliminary experiments that used different doses of radiation, namely, 2, 8 and $25 \mathrm{~Gy}$, a dose of 8 Gy was chosen, because no greater effect was observed when a dose of 25 Gy was used.

\subsubsection{Alterations in chemokine gene-expression in the hepatocytes post-gamma irradiation}

The findings from a previous study showed that gamma-radiation had no direct effect on hepatocyte viability (Christiansen et al. 2004), which suggests that these cells are resistant to the direct action of gamma-radiation. The hepatocytes were examined with respect to chemokine gene expression because they comprise $70-80 \%$ of the cytoplasmic mass of the liver (Grisham et al. 1975), and, when stressed, they can induce several pro-inflammatory mediators (Sheikh et al. 2006a). Upregulation of the CXCL2 (3.3 \pm 0.56 -fold) and CXCL5 $(1.56 \pm 0.1$-fold $)$ genes was detected in the rat parenchymal cells 1 hour after irradiation. The expression of MCP-1/CCL2 gene did not show an obvious change after irradiation, but the CXCR2 (7.7 \pm 1.6 -fold) gene expression was upregulated in the hepatocytes 1 hour after irradiation. The CXCL1 and CCR2 genes were rapidly downregulated at 1 hour and 3 hours after irradiation, respectively, with further declines occurring for up to 24 hours in the irradiated hepatocytes (Fig. 18a, b). Neither the IL-1 beta nor the IL-6 genes were detectable in either the control or the irradiated liver parenchymal cells. 
a) CXC-chemokines

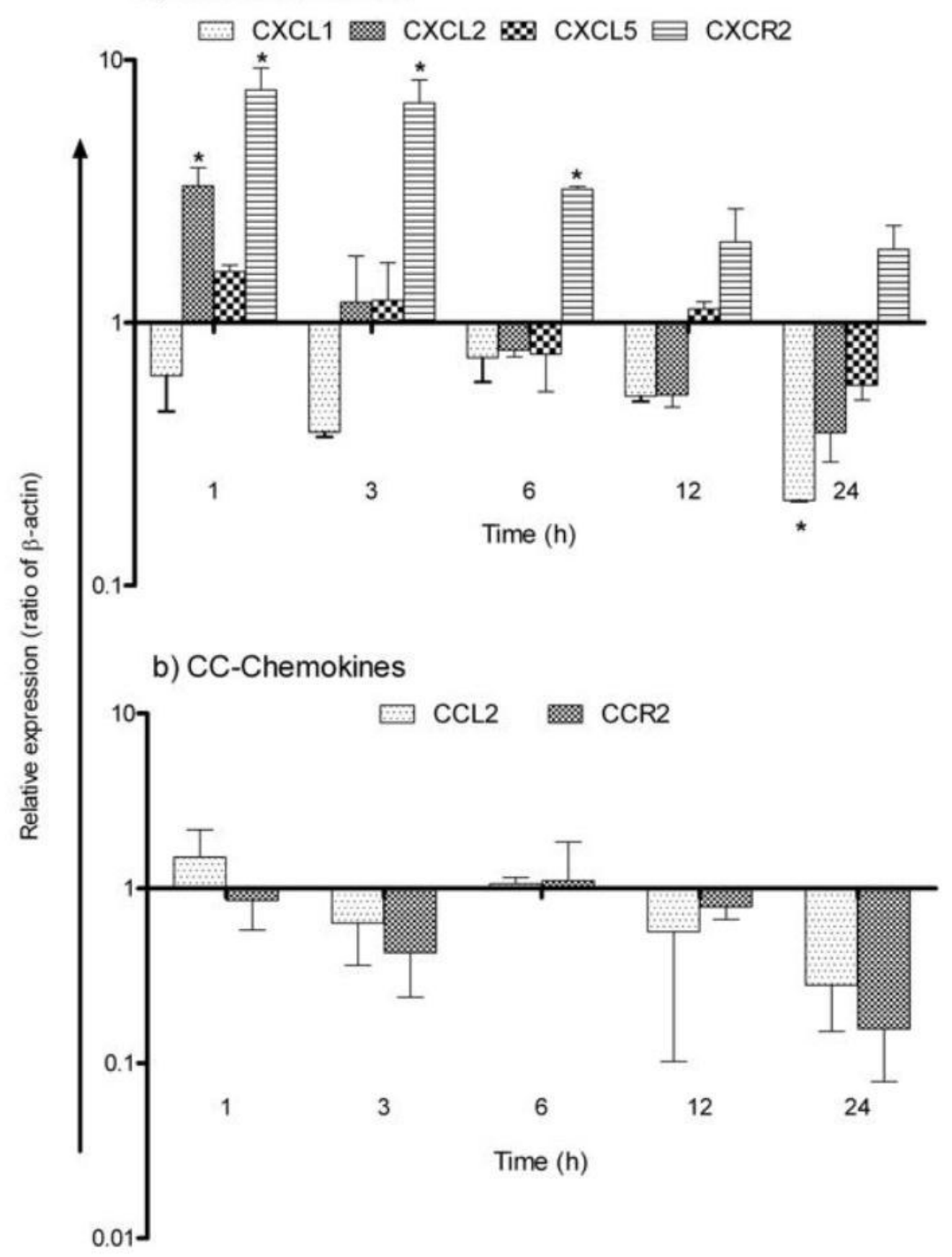

Figure 18: Changes in the levels of messenger ribonucleic acid (mRNA) expression measured by real timepolymerase chain reaction for the (a) CXC chemokines and (b) $\mathrm{CC}$ chemokines at different time points in hepatocytes that were administered radiation at a dose of $8 \mathrm{~Gy}$ relative to the sham-irradiated control hepatocytes. The data are presented as fold changes. Normalisation of the RT-PCR was achieved using the housekeeping genes, namely, beta-actin and ubiquitin. The control value was set at one. The data represent the means \pm the standard errors of the means of three experiments in duplicate, and they were compared with those of non-irradiated cells served as control at each time point $\left({ }^{*} P<0.05\right.$, based on a one-way analysis of variance).

\subsubsection{Changes in chemokine and cytokine gene expression in the liver myofibroblasts after gamma-irradiation}

As described previously, the migration of the neutrophils was only seen around the portal vessels where they were in direct contact with the LMFs. Furthermore, no increase in the numbers of mononuclear phagocytes was detected after gamma-radiation, and radiation is 
known to damage endothelial cells (Maj et al. 2003). The LMFs were isolated and irradiated. In the isolated LMFs, the MCP-1/CCL2 showed a constitutive gene expression which was greater than that in the irradiated liver tissue. The irradiation of the LMFs increased the level of MCP-1/CCL2 (5.7 \pm 1.7-fold) gene expression, and it led to the detection and further increase of other chemokines and cytokines, including IL1 beta and IL-6.

The levels of CXCL1 $(4.8 \pm 0.42$-fold $)$ and CXCL2 $(3.2 \pm 0.44$-fold $)$ mRNA were upregulated quickly and early in the LMFs 1 hour after irradiation. The upregulation of CXCL5 mRNA ( $3.2 \pm 0.44$-fold) was detected in the LMFs at 3 hours after irradiation. The CXCR2 and the CCR2 genes were not detectable in either the control or the irradiated LMFs (Fig. 19).

Therefore, the LMFs were the major source of the chemokines in the liver after irradiation, especially CCL2, which was constitutively expressed by these cells and it was upregulated after irradiation. No major changes were observed in the hepatocytes after irradiation.

\subsection{Comparisons of the chemokines' and cytokines' cycle threshold values in the liver and in the isolated cells}

While the increases in the levels of mRNA specific to particular chemokines and cytokines were indicative of the alterations in expression of single genes that were upregulated after irradiation of liver tissues and cells that had been isolated from the liver, comparisons of the cycle threshold $(\mathrm{Ct})$ values provided indirect indications of the relative levels of gene expression for the chemokines, chemokine receptors and cytokines in the liver tissue, hepatocytes and in the LMFs before treatment. Furthermore, it may provide additional insights into the reasons underlying the discrepancies between the magnitudes of the changes induced by gamma-irradiation in vivo and those induced by irradiating single cell populations in vitro. The $\mathrm{Ct}$ values showed that the relative abundance of specific mRNA in the liver tissue was lower, but that the kinetics of the declines in the $\mathrm{Ct}$ values, that is, the increases in the amounts of specific mRNA, were similar. 


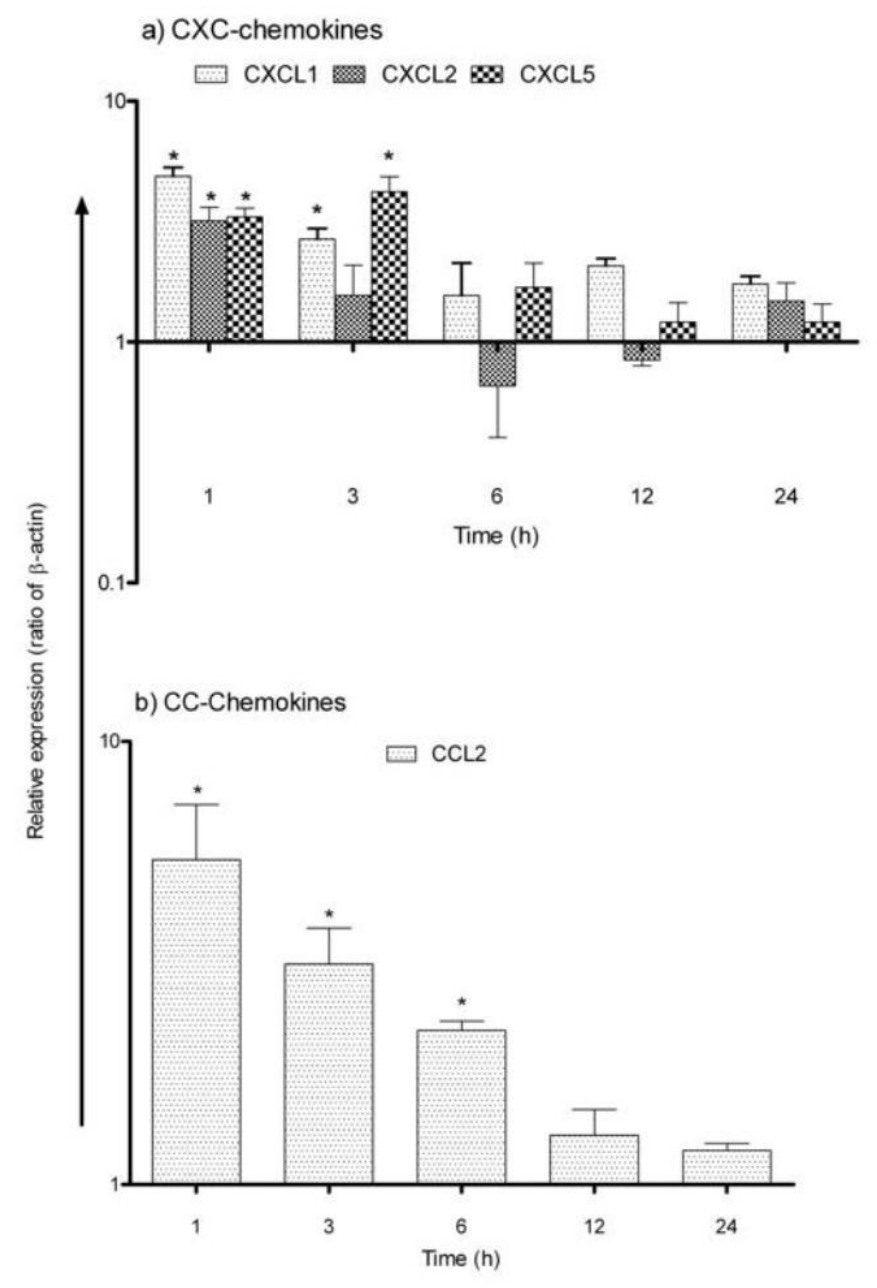

c) Cytokines

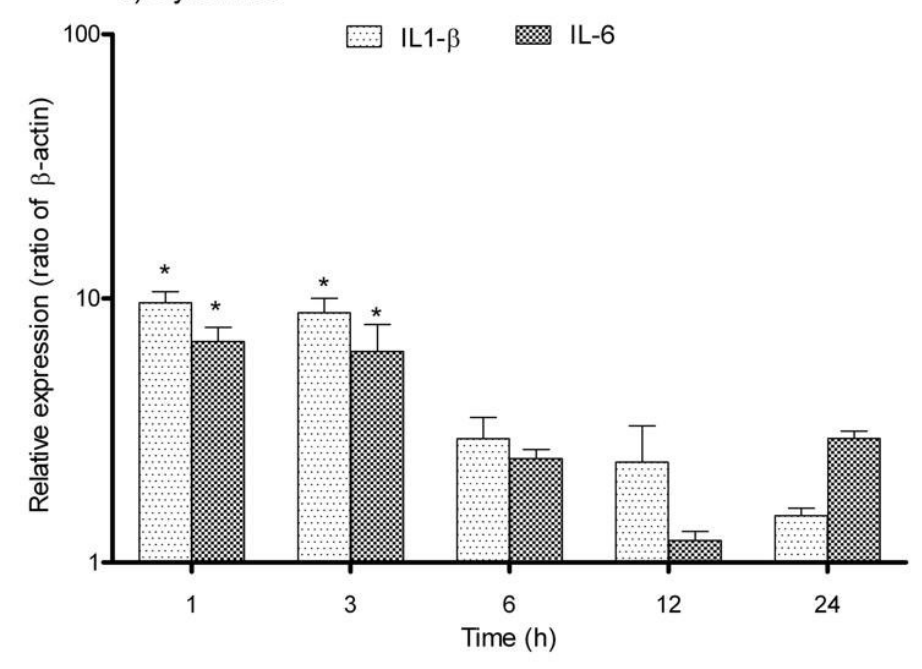

Figure 19: Changes in the levels of messenger ribonucleic acid (mRNA) expression for (a) the CXC chemokines, (b) the CC chemokines and (c) interleukin (IL)- 1 beta and IL-6 in liver myofibroblasts (LMFs) at different time points following irradiation at a dose of 8 Gy compared with sham-irradiated control cells. The 
real time-polymerase chain reaction data that are presented as fold changes in the mRNA expression levels were normalised using two housekeeping genes, namely, beta-actin and ubiquitin $\mathrm{C}$, and the control value was set at one. The data are presented as means \pm standard errors of the mean from three separate experiments carried out in duplicate, and these were compared with those from sham-irradiated cells at each time point $(* P<0.05$, according to a one-way analysis of variance).

Abbreviation: IL, interleukin.

The CXCL2 and CXCL5 genes were constitutively expressed in isolated untreated LMFs with the highest mRNA expression in these cells, followed by hepatocytes and liver tissue. In contrast, the mRNA-level of CXCL1 gene was highest in the untreated hepatocytes, followed by LMFs and the hepatic tissue. The constitutive level of the chemokine genes was low in the liver tissue, and irradiation upregulated the expression of the chemokine genes by about 100-fold in the liver tissue and it upregulated the expression of the chemokine genes by about 10-fold in the LMFs, which was associated with a strong constitutive expression of these genes in isolated LMFs. The transcriptional level of the CXCL1 was higher in the untreated control hepatocytes $(35 \pm 5.2$-fold $)$ and at 3 hours $(21 \pm 3.1$-fold $)$ after the irradiation of the hepatocytes, and it was higher in the untreated control LMFs $(11.5 \pm 2.2-$ fold) and at 3 hours $(33.4 \pm 7.1$-fold $)$ after the irradiation of the LMFs, relative to shamirradiated livers. The CXCL2 gene was more strongly expressed in the untreated control hepatocytes $(15 \pm 1.2$-fold) and at 3 hours $(29 \pm 3.1$-fold $)$ after the irradiation of the hepatocytes, and it was higher in the untreated control LMFs (98 \pm 17 -fold) and at 3 hours $(565 \pm 97.1$-fold) after the irradiation of the LMFs compared with the non-irradiated control livers. The mRNA-level of the CXCL5 gene was higher in the untreated control hepatocytes $(5 \pm 1.2$-fold $)$ and at 3 hours $(9 \pm 3.1$-fold) after the irradiation of the hepatocytes, and it was higher in the untreated control LMFs (65.5 \pm 8.2 -fold) and at 3 hours (668.4 \pm 64 .6-fold) after the irradiation of the LMFs compared with the non-irradiated control livers (Fig. 20). Of the chemokine genes, the MCP-1/CCL2 gene was constitutively expressed and, compared with the sham-irradiated control livers, its level of expression was highest in the untreated control LMFs $(229 \pm 18$-fold $)$, followed by its expression in the untreated control hepatocytes $(2 \pm$ 0.18 -fold). The expression of the gene for MCP-1/CCL2 increased in the LMFs at 3 hours after irradiation (982 \pm 78 -fold) and in the liver (Figs. $15 \& 20$ ) after irradiation, compared with the sham-irradiated livers, but no change was evident in the irradiated hepatocytes. The level of CXCR2 gene expression was higher in the isolated hepatocytes than in the sham- 


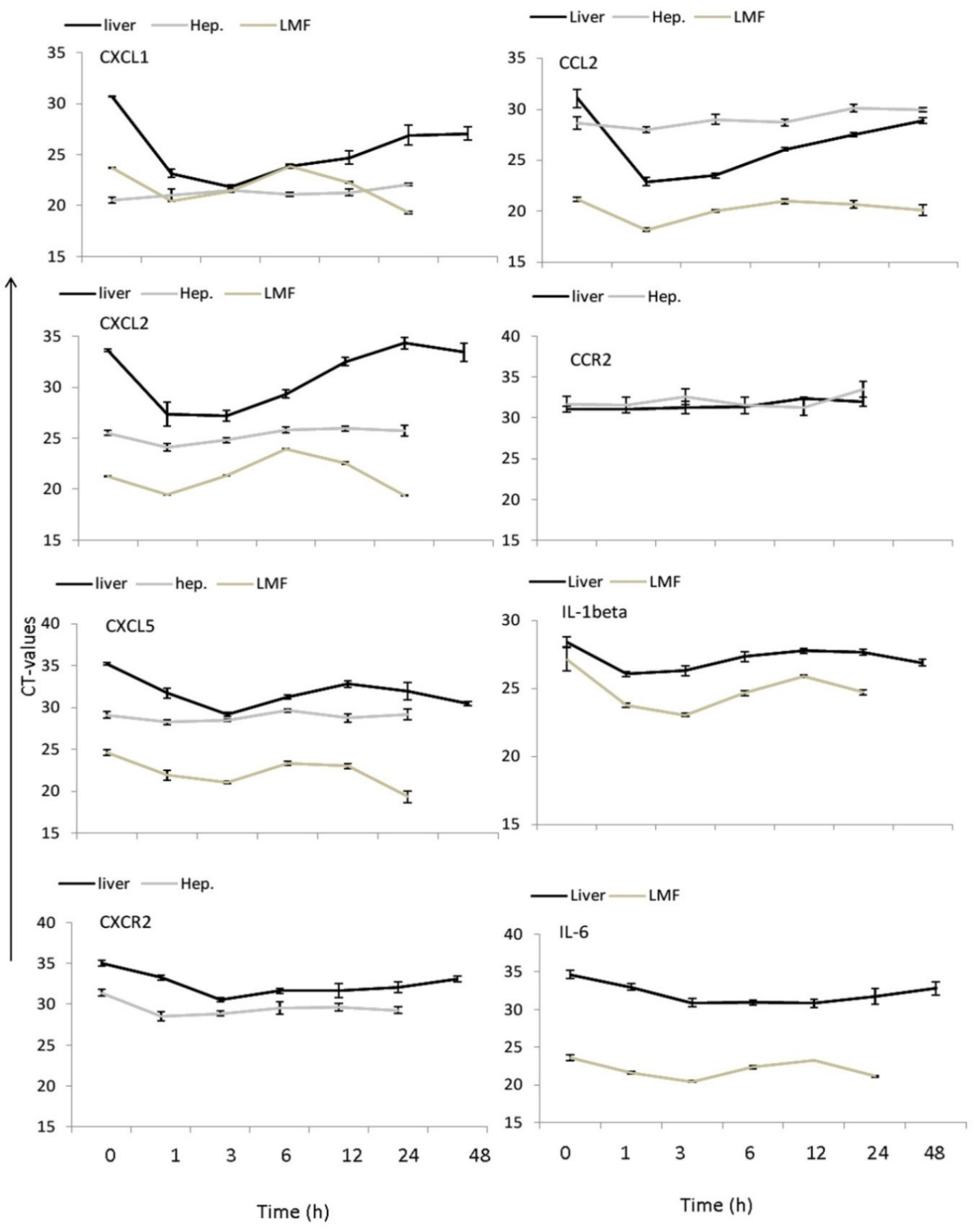

Figure 20: The declines in the cycle threshold (Ct) values for CXCL1, CXCL2, CXCL5, CCL2, CCL7, CXCR2, CCR2, IL-1 beta and IL-6 in irradiated and sham-irradiated control rat livers, hepatocytes and liver myofibroblasts. The data were obtained from real-time polymerase chain reaction analysis of the total ribonucleic acid extracted from control and irradiated livers and from isolated liver cells after gamma irradiation. The data are representative of three experiments performed in duplicate, and the means \pm the standard errors of the means are shown for each time point.

Abbreviations: Ct, cycle threshold; IL, interleukin; Hep, hepatocytes; LMF, liver myofibroblasts. 
irradiated liver tissue whereas no such difference was detected in the expression of the CCR2 gene between the isolated hepatocytes and the sham-irradiated liver tissue. The levels of gene expression within the isolated hepatocytes and within the LMFs differed considerably, but some chemokine genes were constitutively expressed. Moreover, the mRNA-level of these genes induced in hepatic tissue (Fig. 14 \& 15) and in the LMFs (Fig. 19) after irradiation, but no considerable increases in the gene expression levels were observed in the isolated hepatocytes (Fig. 18). Similar observations were apparent in relation to mRNA-levels of the IL-1 beta and IL-6 genes. Compared with the sham-irradiated control liver tissue, the untreated control LMFs displayed a high IL-1 beta (3.6 \pm 0.7 -fold) gene expression level that increased at 3 hours (13.4 \pm 1.2 -fold) after irradiation, and they showed a very high level of expression of the IL-6 gene (159.6 \pm 32-fold) that increased at 3 hours after irradiation (206 \pm 51-fold). The CXCR-2 and CCR2 genes were not detected in the control or in the irradiated LMFs (Fig. 20). 


\section{DISCUSSION}

\subsection{Recent progress in cancer therapy}

The introduction of modern technologies to the medical field has revolutionised treatment options for cancer over the last few years. The main achievements have been associated with improvements in the quality of patients' lives and reducing patients' disease loads. Progress has been made not only with respect to a wide range of monotherapies, but also in relation to combined therapies. Indeed, multi-therapy approaches target the numerous characteristics associated with the biology of cancer, and several of these approaches have been applied to achieve different goals in the hope that from these strategies outstanding solutions will emerge that can treat patients who have cancer that is in its advanced stages (Kamrava et al. 2009).

\subsubsection{Advances in radiation therapy}

Radiation therapy administered in combination with immunotherapy has shown some success, and the effectiveness of this form of combined therapy has been observed in preclinical and clinical settings (Fig. 21).

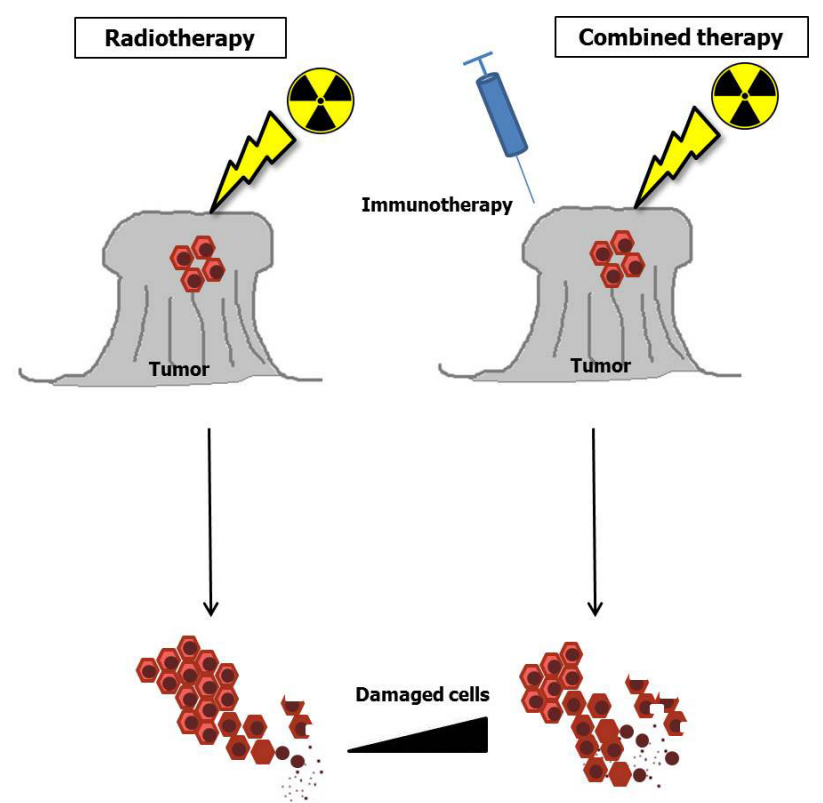

Figure 21: The use of radiotherapy in combination with immunotherapy and anti-angiogenic agents (based on a suggestion by Kamrava et al. 2009).

However, a significant effort is required to increase the success of combined therapies by reducing their side effects. Hence, incorporating other interventions into therapeutic 
combinations may improve tumour treatment (Kamrava et al. 2009). Radiotherapy can be applied to those patients who cannot undergo surgery, and it can be delivered to patients with greater accuracy compared with other therapeutic approaches, including surgery (Dawson and Guha 2008; Mathurin et al. 1998; Seong et al. 2003).

Radiotherapy is used to treat primary and secondary tumours, and there are different forms of radiotherapy, including low-dose whole liver radiotherapy. Of the other types of radiotherapy, confocal local radiotherapy and stereotactic body radiotherapy are the most notable (Dawson and Guha 2008; Hilgard et al. 2009; Park et al. 2005; Seong et al. 2003; Stubbs and Wickremesekera 2004). Clinically, fractionated radiotherapy is the most applied form of radiation therapy, and it has been relatively successful because the radiotoxicity can be controlled, and this is the main limiting factor associated with radiotherapy. The application of this therapy is based on the radiobiological assumption that the mechanisms required to repair reversible damage are more effective in healthy cells than they are in cancer cells (Gregoire 2005).

Hepatic metastases are common in patients with gastrointestinal, breast and lung cancers. Selective ablative radiotherapy is applied to patients who are not candidates for resection, and it aims to achieve better internal control and to improve the long-term survival of patients. This therapy seems to be a better substitute for patients with colorectal carcinomas and for those who have non-colorectal carcinomas that have metastasised to the liver (Dawson and Guha 2008; Stubbs and Wickremesekera 2004).

Toxicity is the main limitation associated with radiotherapy, and the side effects of radiotherapy are both acute and long term. Since the responses of the different organs and tissues to radiotherapy vary, the tolerance dose must be determined for each organ in addition to the factors that cause injuries and induce repairs after radiation therapy (Hawkins and Dawson 2006; Hilgard et al. 2009; Seong et al. 2003; Sharplin and Franko 1989).

\subsection{Radiation-induced liver injury and disease}

Clinical and pathological investigations have shown that RILD is a serious consequence of hepatic radiotherapy (Cheng et al. 2004; Reed and Cox, 1966; Shim et al. 2007). Veno-occlusive disease is a common outcome from RILD, and it is characterised by the obstruction of the small veins in the liver's central portion. Hence, liver enlargement is a characteristic feature of veno-occlusive disease. The obstructions in the veins reduce the blood supply within the liver, which causes hepatic cell damage. Moreover, after radiation 
therapy, transforming growth factor-beta (TGF-beta) is produced (Anscher et al. 1990; Dawson and Guha 2008) which further activates the hepatic stellate cells that could induce hepatic fibrogenesis (Dawson and Guha 2008; Sempoux et al. 1997). RILD patients have high AP levels, which indicates that the hepatocytes as well as the biliary cells are disrupted by radiotherapy (Dawson and Guha 2008; Lawrence et al. 1995). As the mechanism underlying the development of RILD has not yet been fully elucidated, it is assumed that both the parenchymal and non-parenchymal cells are affected by radiation.

Since systematic studies on humans are not possible, several animal models have been established to facilitate investigations into radiation-induced stress. For example, experimental mouse models that have involved the irradiation of organs other than the liver have been established using partial (el-Khatib et al. 1983) and total body (Cui et al. 2002) irradiation. Accordingly, to investigate the consequences of radiation on healthy tissue, a rat model of selective percutaneous liver irradiation was established by Christiansen et al. (2006), which was used in this study.

Irradiated livers are characterised histologically by the presence of steatosis in the periportal hepatocytes, which begins to develop between 24 hours and 48 hours after irradiation (Christiansen et al. 2006). Using the same rat model, alterations in the mRNA level of the genes coding for the adhesion molecules that are largely involved in cell transmigration processes during inflammation were observed (Moriconi et al. 2009). However, the expression of the IFN-gamma gene was downregulated, and no obvious inflammatory infiltrates were detected within the liver parenchyma using light microscopy.

\subsubsection{Markers of inflammation and hepatic injury}

The tissue stress caused by radiation leads to the release of inflammatory cytokines and chemokines (Christiansen et al. 2006; Ramadori et al. 2008), which could be involved in hepatic cell transmigration process (Ramaiah and Jaeschke 2007). In addition to elevating the levels of the AP-mediators, including IL-1 beta, IL-6 and TNF-alpha (Christiansen et al. 2007b), gamma-irradiation additionally induced the very early upregulation of the genes for several pro-inflammatory chemokines, including MCP-1/CCL2, MIP-3 alpha/CCL20, MIP-3 beta/CCL19, IL-8/CXCL8, MIG/CXCL9, IP-10/CXCL10 and ITAC/CXCL11 (Moriconi et al. 2008).

Correspondingly, the early secretion of CXCL2 into the serum was detected following liver irradiation in the present study (Fig. 5). Our previous and current findings suggest that 
early increases in the serum levels of chemokines and cytokines could be the response of stressed liver cells, particularly the hepatocytes, to radiation. Interestingly, radiation-triggered elevations in the serum levels of these mediators were observed before the migration of the leukocytes into the liver. These findings indicate the initiation of an inflammatory response that could be followed by liver damage.

To determine whether selective liver irradiation induces hepatic damage, the levels of liver enzymes (ALT, AST) and AP in the serum was measured, which are considered adequate for detecting liver injury. Increases in the levels of these enzymes have been reported in association with several liver diseases (Rej 1989; Sheikh 2006; Zamara et al. 2007).

Of the liver enzymes that showed elevations in their serum concentrations following irradiation in the current study, only the increase in the AST level was significant (Fig. 5). ALT is a marker of hepatic damage, and it is found exclusively in the cytoplasm of cells. In contrast, AST is present in the cytoplasm, which comprises $20 \%$ of its total activity, and in the mitochondria, which comprises $80 \%$ of its total activity (Rej 1989). Radiation might have a higher impact on the numerous large mitochondria that reside within the hepatocytes, which would lead to a greater release of AST than ALT.

\subsubsection{Leukocytic responses to hepatic irradiation}

Once mild hepatic damage was evident, the next step was to investigate the effect of irradiation on healthy liver by analysing the different regions of the liver tissue to detect the presence of leukocytes in relation to the chemokines known to participate in the recruitment of these leukocytes. A brisk recruitment of neutrophil granulocytes was observed around the portal vessels of the liver after a single high dose (25 Gy) of gamma-irradiation (Fig. 6). Interestingly, these granulocytes were only detectable in the portal area in the vicinity of hepatic Thy $-1^{+}$myofibroblasts (LMFs) (Fig. 10), and they were not present close to sinusoids or the central vein. The levels of $\mathrm{ED}^{+}$or $\mathrm{ED} 2^{+}$mononuclear phagocytes did not increase at any time after irradiation (Fig. 8).

In classical inflammation, macrophages follow the neutrophils to the injured site. The absence of an alteration in mononuclear phagocytes number in the same area in which the increase in granulocytes number was noticed following irradiation in the present study could be associated with radiation-induced structural modifications within these cells that could weaken the defence response. It is possible that mononuclear phagocytes lose their normal 
defence functions after irradiation and that they become unable to increase in number. Furthermore, the post-irradiation releases of the inflammatory mediators that recruit mononuclear phagocytes may not reach the threshold levels that are required to mobilise the macrophages, and inflammatory mediators regulate leukocyte production in the bone marrow. Radiation may also alter the levels of gene expression associated with the inflammatory mediators and adhesion molecules that are required for macrophage transmigration, thereby preventing its occurrence. Moriconi et al. (2008) showed the presence of leukopenia, in the same rat model which supports this finding. The reasons underlying the absence of changes in the macrophage numbers at the injured site following irradiation and the consequences of this phenomenon are topics for future investigation (Moriconi et al. 2008).

Tello et al. (2008) described radiation-induced cell damage that is precipitated by many factors. Together with liver transaminase data, the findings from this experiment suggest that irradiation per se causes only a mild damage to the cells, which could, however, be augmented in the presence of neutrophils.

\subsubsection{Gene expression responses to hepatic insults}

As technology has progressed, new methods, including gene array analysis, have been established that can detect changes at the RNA level on a large scale. Indeed, gene array analysis has been used to study alterations in the genes at the mRNA level, which could play a role in tissue injury caused by irradiation (Christiansen et al. 2006; Kruse et al. 2004). In the current investigation, microarray analysis determined that 31 genes were upregulated in the liver in vivo, including Abcb1b and Ccng1, and that 24 genes were downregulated in the liver 6 hours after the rats had been administered single-dose liver focused gamma-irradiation at 25 Gy (Tables 3 \& 4). The upregulated genes are mainly involved in the cell cycle, DNA synthesis and in transport processes within the liver. However, the microarray gene expression data showed that only a few of the genes detected in the irradiated livers were differentially expressed compared with the livers from the control animals. Therefore, the radiation-induced expression of chemokine-genes had to be studied using the RT-PCR, which is a more sensitive and specific method. The differences in the expression profiles may be a consequence of the different methods of detection used. The RT-PCR is considered to be more sensitive fluorescence-based mRNA detection and quantification method, and it can detect smaller amounts of RNA than the hybridisation-based detection methods (Fehr et al. 2000) that are used in microarray gene expression analysis. This is in accordance with a 
previous investigation in which the authors determined that genes that have very high or low levels of expression, or those that have larger distances between the locations of the PCR primers and the microarray probes tend to have reduced levels of agreement between microarray analyses and the RT-PCR (Etienne et al. 2004).

\subsection{Roles of inflammatory mediators and of endothelial cells in inflammatory cell transmigration}

Inflammatory cells must undergo activation or priming by inflammatory mediators that include cytokines, a large number of chemokines and other biologically active molecules (Ramaiah and Jaeschke 2007), before they adhere to the vessel walls and transmigration can occur. Therefore, the expression of the genes for additional chemokines and the presence of inflammatory cells within the tissues were investigated.

In the current study, the RT-PCR determined that a single dose of gamma-irradiation rapidly upregulated the expression of the genes for CXCL1, CXCL2, CXCL5, CXCL8, MCP1/CCL2, MCP-3/CCL7 and for the CXCR2 receptor in the liver (Figs. $14 \& 15$ ). The upregulation of these genes was observed together with higher neutrophil numbers around the portal vessel walls, but not in the sinusoids within the parenchyma. This may support the notion that the mesenchymal cells of the sinusoidal wall, that is, the hepatic stellate cells, differ from the Thy- $1^{+}$mesenchymal cells in the portal vessels and in the portal area (Dudas et al. 2009), and may explain why the granulocytes do not pass through the fenestrae of the sinusoidal endothelial cells.

Although the role of the endothelial cells in cell recruitment may be minor, which was suggested by Gaugler et al. (1998) who irradiated these cells in vitro and found that chemokine gene expression was not upregulated, these cells are important for the first phase of leukocyte attachment and rolling (Petri et al. 2008). Furthermore, damage to endothelial cells is a primary event that is caused by radiation (Maj et al. 2003).

\subsection{Roles of the chemokines and the liver myofibroblasts in inflammatory cell recruitment}

Immunohistology and LCM determined, for the first time, that the localization and upregulation of MCP-1/CCL2 was confined to the portal area, but was absent in the vicinity of sinusoids of hepatic parenchyma (Figs. $11 \&$ 16). Similar expression patterns were apparent for the CXCL1 and CXCL8 genes, and the CXCL1 gene additionally showed a high 
level of mRNA in the parenchyma (Fig. 16). The upregulation of these genes was accompanied by neutrophil recruitment that was restricted to the portal area; hence, the neutrophils did not appear to be recruited into the parenchyma, suggesting that this process could not be initiated by the induction of a single chemokine. The findings from the LCM analysis directed a key role for MCP-1/CCL2 in the migration of neutrophils into the portal area. MCP-1/CCL2 has been reported to attract neutrophils by inducing the production of the leukotriene $B_{4}$ in a septic peritonitis mouse model (Matsukawa et al. 1999). Additionally, the neutralisation of MCP-1/CCL2 not only reduced the accumulation of monocytes within the intestinal muscularis, but it also significantly reduced the infiltration of polymorphonuclear leukocytes in an LPS-induced model of inflammation (Turler et al. 2002). ROS produced as a consequence of ischemia-reperfusion injury significantly increased the level of MCP-1/CCL2 mRNA in the heart (Morimoto et al. 2008), and the same effect has been shown in rats administered the hepatotoxin, carbon tetrachloride (Czaja et al. 1994), and in mice that underwent whole lung irradiation (Ao et al. 2009). IL-8/CXCL8 is of crucial importance for the recruitment of granulocytes to different tissues (Harris et al. 1996). In fact, Sheikh et al. (2006a) found a massive upregulation of the IL-8/CXCL8 gene in the liver of rats that had been treated with turpentine oil intramuscularly, but granulocyte infiltrations into the liver was not detected.

To explore the link between neutrophil recruitment and MCP-1/CCL2 in the current setting, a monoclonal antibody against MCP-1/CCL2 was intraperitoneally administered to animals 30 minutes before irradiation and the animals were sacrificed at the time when previous experiments had indicated that the highest number of neutrophils would be present. The main changes at the RNA level were observed in relation to IFN-gamma and of IP10/CXCL10, which is one of its targets (Fig. 17), but no changes were observed with respect to the number of neutrophils recruited into the portal area. Hence, MCP-1/CCL2 may have an inhibitory effect on IFN-gamma gene expression, which has been observed in $\mathrm{T}$ cells (Hogaboam et al. 1998), and the increase in the level of IP-10/CXCL10 gene expression may have been compensating for the decline in MCP-1/CCL2 availability, because IP-10/CXCL10 may also participate in neutrophil recruitment (Taub et al. 1996). However, it is possible that a higher amount of antibody administered earlier or later, or before or during irradiation may inhibit neutrophil recruitment into the portal field. These data suggest that the administration of several antibodies that are specific for different chemokines is necessary to reduce neutrophil migration into the portal area. 
The LMFs were studied to determine the cellular source of the upregulated chemokines and cytokines within the portal area, because they are an important source of chemokines and cytokines, including IL-6 (Knittel et al. 1999; Marra 2002), and the portal structures stained positively for MCP-1/CCL2. LMFs that had been isolated from the liver and cultured, and were examined after the first passage showed high levels of constitutive gene expression for the chemokines that were upregulated in the whole liver and within the portal area following irradiation. The irradiation of the LMFs induced the levels of gene expression for CXCL1, CXCL2, CXCL5, MCP-1/CCL2, IL-1 beta and IL-6 (Fig. 19). Like the LMFs, the isolation, culturing and irradiation of the hepatocytes was performed. The hepatocytes are the main population of cells within the liver and are considered to play, together with the Kupffer cells, a key role in hepatic host defense under stress conditions. Irradiated hepatocytes showed an upregulation of the expression of CXCL2 gene and its receptor (Fig. 18), suggesting that these cells are least affected by radiation. A minor role for the Kupffer cells in neutrophil migration can be assumed in the current setting as radiationinduced apoptosis of Kupffer cells has been described (Tello et al. 2008). This information and the current results suggest that the LMFs could be a key target for radiation and the main source of the factors that influence neutrophil migration through the vessel walls.

As LMFs constitutively express most of the chemokine and cytokine genes that are upregulated by administering gamma-irradiation to the liver and to isolated cells (Figs. 19 \& 20), the first steps in the migration of the neutrophils into the portal area of liver, at least, could be attributable to the LMFs.

The data presented here taken together with the findings from previous studies carried out by our research team, suggest that the upregulation of the genes for several chemokines, which occurs mainly in the LMFs, may be necessary to recruit inflammatory cells to the local area, and that this begins around the walls of the portal vessels. The absence of granulocytes in the liver sinusoids may be explained by the absence of such a vessel wall in this area. In addition, the comparably high radioresistance of healthy hepatocytes is in accordance with the observation of mild hepatic damage after irradiation in our model.

\subsection{Post-irradiation reactive oxygen species production and hypoxia}

One of the mechanisms that may underlie the cellular and tissue damage that occurs after irradiation is the production of ROS by irradiated cells and tissues (Leach et al. 2001; Riley 1994), which is linked to higher releases of molecules that include superoxide radicals, 
hydroxyl radicals, and hydrogen peroxide, as a consequence of oxidative stress (Riley 1994). Furthermore, a link between ROS production following whole body exposure to gammairradiation and hepatic injury has been described in mice (An et al. 2004; Riley 1994). It is known that oxidative processes are activated in the mitochondria in response to the stress caused by ionising radiation. Consequently, a local release of calcium ions $\left(\mathrm{Ca}^{2+}\right)$ occurs that is absorbed by the nearby mitochondria and they undergo the mitochondrial permeability transition. The released $\mathrm{Ca}^{2+}$ is passed from one mitochondrion to another to transfer the signal. The increase in the $\mathrm{Ca}^{2+}$ level can lead to the production of ROS within the mitochondria (Leach et al. 2001; Vercesi et al. 1997). Given the indication of mitochondrial damage in our model, this process may have been connected to the liver injury that followed irradiation.

Earlier findings have shown that radiation-induced ROS can trigger the nuclear factorkappa B (NF-kB) pathway, which could further activate the genes for the pro-inflammatory mediators, including chemokines, cytokines and adhesion molecules (Droge 2002; Karin and Delhase 2000; Leach et al. 2001; Lin et al. 2009; Luo et al. 2005).

ROS-dependent signalling regulates several critical processes, including the activation of inflammasomes, which are multiprotein complexes that promote the induction of caspase-1 by releasing pro-inflammatory cytokines (Franchi et al. 2009). Active caspase-1 may be necessary for the activation and maturation of pro-IL-1 beta and pro-IL-18 through cleavage. Whereas biologically mature IL-1 beta is associated with several immune processes, including attracting inflammatory cells, IL-18 is required for IFN-gamma production and to trigger the increase in the cytolytic activity of natural killer cells (Arend et al. 2008).

Indeed, radiation-induced injury in the vascular endothelium may reduce levels of oxygenation in healthy tissue and this could lead to hypoxia (Kiani et al. 2003; Stone et al. 2002). Among other effects, radiation-induced functional and structural modifications in the microvascular network have been reported (Stone et al. 2002), which regulates the delivery of oxygen to the tissues, and it can induce changes in the regulatory mechanisms within the microvascular network (Gaber et al. 2003) and in the inflammatory cascade (Kiani et al. 2002). The microvasculature changes caused by radiation may have a marked effect on tissue oxygenation and lead to the development of acute and/or chronic hypoxia. The subsequent reduction in the oxygen tension within the tissues activates the signalling pathways that support cell survival and are largely mediated by transcription factors such as hypoxia inducible factor-1 (HIF-1) (Semenza et al. 2000). Furthermore, hypoxia-induced ROS could 
stimulate chemokine and cytokine synthesis and upregulate the genes associated with the adhesion molecules (Bremer et al. 1994; Ghezzi et al. 1991; Lin et al. 2009). Tumour oxygenation and its role in tumour progression and therapy have been studied extensively (Masunaga and Ono 2002; Mizumoto et al. 2002), but the influence of hypoxia on the development of radiation damage is poorly understood in normal tissues (Stone et al. 2002). Radiation-induced apoptosis or cell cycle arrest through radiation-induced hypoxia could be proposed as a mechanism to explain the cascade of events that occurs following irradiation and causes long-term tissue damage ( $\mathrm{Li}$ et al. 2001; Vujaskovic et al. 2001).

In addition, ionising radiation destroys cells by damaging their DNA. The mechanisms underlying DNA damage are well understood. Cellular DNA damage can be caused directly through ionisation or indirectly through the radiolysis of the water molecules in the surrounding tissue, that produces hydroxyl radicals and initiates DNA radical formation. Under hypoxic conditions, tumour cells escape this process, which may explain why cancer cells are radioresistant (Wouters et al. 2007).

\subsection{The tumour microenvironment and the impact of radiotherapy}

The radioresistant nature of many tumours is one of the issues that has contributed to the minor success of radiotherapy in treating cancer. The radioresistance of tumours is associated with the TME, and the hypoxic environment (Karar and Maity 2009; Moeller et al. 2004).

The TME comprises endothelial cells, fibroblasts, adipocytes, mesenchymal cells and stem cells, extracellular matrix components, cellular and humoral components of the immune system, and other factors that include ROS, cytokines and growth factors (Bhowmick et al. 2004). The constituents of the TME interact not only with the tumour cells, but they also interact with each other, and, depending upon the circumstances, they can exert promalignancy or anti-malignancy effects (Witz 2009). Together, the tumour cells and the TME define the tumour's survival, growth, proliferation, metastatic potential and response to therapy (Mbeunkui and Johann, 2009).

The TME has not received much attention in the past, because radiation therapy was thought to act directly on tumour cells only. However, the significance of the role of TME has gradually been recognised. Stromal, vascular and immunological changes are observed in the TME following radiation therapy and evidence is accumulating that suggests that the irradiation of tumours may also induce the release of inflammatory cytokines that modify the 
profile and function of the leukocytic infiltrates (Moeller et al. 2004; Spaeth et al. 2008). Postirradiated tumours are characterised by greater levels of hypoxia, upregulated hypoxia inducible factor-1 signalling, the expression of a diverse range of cytokines (Moeller et al. 2004) and a greater recruitment and influx of bone marrow-derived tumour-associated macrophages that can promote angiogenesis (Tjiu et al. 2009).

Hence, it has become clear that the effectiveness of radiotherapy may depend not only on the sensitivity of the tumour cells, but also on the sensitivity of the cells within the TME.

Like these findings, those from the present work propose that radiation alters the microenvironment of healthy cells. Moreover, these alterations are supplemented by an activated hepatic immune response, which gives rise to the possibility of a more efficient immunotherapeutic approach against tumour cells through the release of anti-tumour mediators and the recruitment of immune cells. Greater understanding of the pathways that lead to the responses of the TME and tumours to radiotherapy might uncover several classes of agents that could increase tumor radiosensitivity through the modulation of the TME (Karar and Maity 2009). Accordingly, an in-depth understanding of the radiation-altered microenvironment of healthy tissues could help to develop protective agents that could enhance targeted radiation doses against tumour tissues.

\subsection{Summary of study results}

In summary, single-dose (unfractionated) gamma-irradiation administered percutaneously to healthy rat livers induces mild liver damage and the transient increase in neutrophil numbers within the portal area only, and it does not induce the recruitment of mononuclear phagocytes. The recruitment of the granulocytes is preceded by rapid and early inductions of several chemokine genes, namely, CXCL1, CXCL2, CXCL5, CXCL8, CCL2 and CCL7, and of the chemokine receptor gene, CXCR2, in irradiated liver tissue and especially in microdissected cells from the structures of the portal area. The induction of these mediators was followed by mild liver damage. The processes underlying neutrophil recruitment, adhesion and transmigration following irradiation may be related to differential chemokine expression within the cells of the portal vessels, and the LMFs seem to be a major source of the chemokines that participate in the migration of neutrophils. The intraperitoneal administration of a single dose of an antibody against MCP-1/CCL2 30 minutes before irradiation did not influence granulocyte recruitment into the portal area, but it altered the expression of some genes within the liver. The induction of the mediators following 
irradiation may have occurred via ROS production. As the tissue damage observed after irradiation was minor, the frequently reported sensitivity of the liver tissue to gammairradiation may be caused by a further reduction in the blood flow within the cirrhotic liver because of the pre-existence of a portal blood bypass. The hepatic damage that is observed after the irradiation of liver metastasis within normal liver tissue may be a consequence of the administration of two noxae, namely, gamma-irradiation and the frequent concomitant administration of chemotherapy.

Normal liver tissue seems to be quite resistant to high, single-dose gamma-irradiation. The processes underlying the radiation-induced damage and repair of tissues appear to differ from those associated with the classical inflammation processes. Further studies on this topic could help to determine the mechanisms underlying RILD and to find ways to protect tissues from radiation-induced damage.

\subsection{Review of the recent literature}

Recently, the mechanisms underlying the cell transmigration process and damage caused by radiation have been partially explored. Hepatic TNF-alpha levels increase after irradiation. In these studies, the roles of the inflammatory mediators, for example, TNF-alpha, and the adhesion molecules, including PECAM-1 and intercellular adhesion molecule (ICAM)-1, have been investigated in vivo and in vitro. The findings from an in vitro study demonstrated the anti-inflammatory effects of infliximab, which is an antibody against TNFalpha that binds and neutralises TNF-alpha, prevented the inhibition of PECAM-1 and the upregulation of ICAM-1 in irradiated U-937 promonocytic cells (Malik et al. 2015) as well as in leukocytes following TNF-alpha treatment (Moriconi et al. 2012). Another interesting finding was the regulation of PECAM-1 through TGF-beta, which indicates that TGF-beta may be an additional therapeutic target that has an anti-inflammatory function (Moriconi et al. 2012). A role for PECAM-1 in inflammatory cell recruitment was further supported when the livers of PECAM-1 knockout mice were irradiated, and a higher number of neutrophils were detected in parallel to elevations in inflammatory mediators, including TNF-alpha and chemokines, compared with wild-type mice (Malik et al. 2015), suggesting that the TNFalpha-regulated reduction in PECAM-1 is an important step in regulating the transmigration processes of inflammatory cells (Neubauer et al. 2008; Privratsky et al. 2010). Furthermore, PECAM-1 knockout mice were more sensitive to radiation, because a higher level of inflammation that was more prolonged was noted in the livers of these animals. Blocking 
TNF-alpha seems to be a useful approach towards alleviating hepatic damage (Malik et al. 2015).

Radiation-triggered fat accumulation within the adipose tissue has been described previously (Jo et al. 2011). A link between the inflammatory mediators, such as TNF-alpha, and the accumulation of fat is well established (Manco et al. 2007). In a related investigation, a drug that inhibited TNF-alpha was administered to wild-type mice before they underwent irradiation to reduce the amount of fat that accumulated in the liver. However, the expected results did not occur and the TNF-alpha inhibitor only marginally inhibited radiation-induced CD36, which is one of the key fat transporter proteins (Martius et al. 2015). The shortcomings of this study may have been associated with the optimisation of the dose required or more time may have been needed for the TNF-alpha inhibitor to control steatohepatitis.

The early diagnosis of radiation-induced effects is a limitation associated with radiotherapy, and efforts have been made in the diagnostics field to detect radiation-induced consequences as early as possible. Recently, a new potential biomarker for radiation-induced hepatic damage, lipocalin-2, has been described (Sultan et al. 2012).

Clinically, single-dose irradiation of the entire liver has not been undertaken to date, because the human liver has been considered radiosensitive, and fractionated doses ( $2 \mathrm{~Gy} /$ day) of irradiation that are administered over longer periods of time (6 weeks) are usually applied to patients. Therefore, there was a need to develop an animal model that could be used for comparisons with clinical settings. Such a model was developed in which rats were administered fractionated irradiation doses of $2 \mathrm{~Gy} /$ day for 6 weeks. This model was compared with a model that received single doses of irradiation. Three months after the administration of the irradiation was terminated, an examination of the liver tissue sections did not reveal any substantial differences between the approaches (Rave-Frank et al. 2013). The absence of any severe long-term consequences following fractionated irradiation or a single-dose of irradiation suggests that this treatment could be used safely in the treatment of liver metastasis if drugs are not administered concomitantly.

Progress has also been made in relation to treating patients with $\mathrm{HCC}$ with different radiotherapy sources and doses. The findings from a recent study in which the investigators administered repeated stereotactic ablative radiotherapy, which is directly administered into the tumor showed the effectiveness of this therapy in patients with HCC and liver cirrhosis and that it had an acceptable level of toxicity (Lo et al. 2014). The main challenges of radiotherapy include targeting tumours with greater accuracy while minimising the effects of 
the irradiation on the surrounding healthy tissues. In a very recent study, carbon ion radiotherapy was compared with stereotactic body radiotherapy with photon beams, and the findings from this study showed more target conformity in association with the carbon ion radiotherapy in the treatment of patients with $\mathrm{HCC}$ and cirrhosis. Accordingly, carbon ion radiotherapy reduces the level of damage in healthy tissues (Abe et al. 2015).

These studies have broadened the scope for the application of radiotherapy to hepatic cancer patients by investigating techniques that lessen the side effects of radiation. Moreover, these reports provide a solid base from which potential targets can be investigated in the future.

\subsection{Future perspectives}

The current study focused on the effect of selective single-dose gamma-irradiation on healthy rat livers, and the data indicate that the rapid induction of inflammatory mediators was followed by the recruitment of granulocytes and mild hepatic injury. This was evident from the presence of granulocytes around the portal vessels and the slight elevation of the serum AST level. The LMFs of the portal vessels' walls seem to be primary site for the local attachment of granulocytes following irradiation.

One of the main conclusions from this study is that blocking only one factor may not be sufficient to control the radiation-induced recruitment of the granulocytes. Therefore, more than one factor or target needs to be inhibited in the liver to prevent radiation-induced damage. Future efforts should aim to optimise the suppression of stress induction in liver cells, for example, by administering anti-cytokine- or anti-chemokine-antibodies as well as antibodies against adhesion molecules, as has been established in the therapy of chronic inflammatory diseases. Likewise, the use of antioxidants, for example, superoxide dismutase or melatonin, could be attempted, which may inhibit the release of cytokines and chemokines and the impact of their releases. The preventive administration of such agents could enable the focused doses of radiotherapy that are applied to liver tumours to be increased. On the other hand, understanding the mechanisms underlying the damaging effects of gamma radiation could help us to use this therapeutic option more effectively to destroy tumour tissues. Clinically, patients with liver cancer could be treated with a single high dose of irradiation rather than fractionated irradiation, which would be effective in terms of cost and time. Using radiotherapy while controlling its side effects will expand the scope of the available curative options for patients with liver cancer. 


\section{REFERENCES}

Abe T, Saitoh J, Kobayashi D, Shibuya K, Koyama Y, Shimada H, Shirai K, Ohno T, Nakano T (2015) Dosimetric comparison of carbon ion radiotherapy and stereotactic body radiotherapy with photon beams for the treatment of hepatocellular carcinoma. Radiat Oncol 10:187

Ajuebor MN, Flower RJ, Hannon R, Christie M, Bowers K, Verity A, Perretti M (1998) Endogenous monocyte chemoattractant protein-1 recruits monocytes in the zymosan peritonitis model. J Leukoc Biol 63:108116

Alati T, Eckl P, Jirtle RL (1989a) An in vitro micronucleus assay for determining the radiosensitivity of hepatocytes. Radiat Res 119:562-568

Alati T, Van CM, Jirtle RL (1989b) Radiosensitivity of parenchymal hepatocytes as a function of oxygen concentration. Radiat Res 118:488-501

An JH, Kim J, Seong J (2004) Redox signaling by ionizing radiation in mouse liver. Ann N Y Acad Sci 1030:8694

Anscher MS, Crocker IR, Jirtle RL (1990) Transforming growth factor-beta 1 expression in irradiated liver. Radiat Res 122:77-85

Ao X, Zhao L, Davis MA, Lubman DM, Lawrence TS, Kong FM (2009) Radiation produces differential changes in cytokine profiles in radiation lung fibrosis sensitive and resistant mice. J Hematol Oncol 2:6

Arend WP, Palmer G, Gabay C (2008) IL-1, IL-18, and IL-33 families of cytokines. Immunol Rev 223:20-38

Baggiolini M (1998) Chemokines and leukocyte traffic. Nature 392:565-568

Benjamini Y, Drai D, Elmer G, Kafkafi N, Golani I (2001) Controlling the false discovery rate in behavior genetics research. Behav Brain Res 125:279-284

Bhowmick NA, Neilson EG, Moses HL (2004) Stromal fibroblasts in cancer initiation and progression. Nature 432:332-337

Boring L, Gosling J, Chensue SW, Kunkel SL, Farese RV, Jr., Broxmeyer HE, Charo IF (1997) Impaired monocyte migration and reduced type 1 (Th1) cytokine responses in $\mathrm{C}-\mathrm{C}$ chemokine receptor 2 knockout mice. J Clin Invest 100:2552-2561

Boring L, Gosling J, Cleary M, Charo IF (1998) Decreased lesion formation in CCR2-/- mice reveals a role for chemokines in the initiation of atherosclerosis. Nature 394:894-897

Bozic CR, Kolakowski LF, Jr., Gerard NP, Garcia-Rodriguez C, von Uexkull-Guldenband C, Conklyn MJ, Breslow R, Showell HJ, Gerard C (1995) Expression and biologic characterization of the murine chemokine KC. J Immunol 154:6048-6057

Bremer C, Bradford BU, Hunt KJ, Knecht KT, Connor HD, Mason RP, Thurman RG (1994) Role of Kupffer cells in the pathogenesis of hepatic reperfusion injury. Am J Physiol 267:G630-G636

Butterfield TA, Best TM, Merrick MA (2006) The dual roles of neutrophils and macrophages in inflammation: a critical balance between tissue damage and repair. J Athl Train 41:457-465

Cannon JG, St Pierre BA (1998) Cytokines in exertion-induced skeletal muscle injury. Mol Cell Biochem 179:159-167

Chandrasekar B, Smith JB, Freeman GL (2001) Ischemia-reperfusion of rat myocardium activates nuclear factor-KappaB and induces neutrophil infiltration via lipopolysaccharide-induced CXC chemokine. Circulation 103:2296-2302

Cheng JC, Wu JK, Lee PC, Liu HS, Jian JJ, Lin YM, Sung JL, Jan GJ (2004) Biologic susceptibility of hepatocellular carcinoma patients treated with radiotherapy to radiation-induced liver disease. Int $\mathbf{J}$ Radiat Oncol Biol Phys 60:1502-1509

Chevret S, Trinchet JC, Mathieu D, Rached AA, Beaugrand M, Chastang C (1999) A new prognostic classification for predicting survival in patients with hepatocellular carcinoma. Groupe d'Etude et de Traitement du Carcinome Hepatocellulaire. J Hepatol 31:133-141 
Chirgwin JM, Przybyla AE, MacDonald RJ, Rutter WJ (1979) Isolation of biologically active ribonucleic acid from sources enriched in ribonuclease. Biochemistry 18:5294-5299

Christiansen H, Saile B, Neubauer-Saile K, Tippelt S, Rave-Frank M, Hermann RM, Dudas J, Hess CF, Schmidberger H, Ramadori G (2004) Irradiation leads to susceptibility of hepatocytes to TNF-alpha mediated apoptosis. Radiother Oncol 72:291-296

Christiansen H, Batusic D, Saile B, Hermann RM, Dudas J, Rave-Frank M, Hess CF, Schmidberger H, Ramadori G (2006) Identification of genes responsive to gamma radiation in rat hepatocytes and rat liver by cDNA array gene expression analysis. Radiat Res 165:318-325

Christiansen H, Saile B, Hermann RM, Rave-Frank M, Hille A, Schmidberger H, Hess CF, Ramadori G (2007a) Increase of hepcidin plasma and urine levels is associated with acute proctitis and changes in hemoglobin levels in primary radiotherapy for prostate cancer. J Cancer Res Clin Oncol 133:297-304

Christiansen H, Sheikh N, Saile B, Reuter F, Rave-Frank M, Hermann RM, Dudas J, Hille A, Hess CF, Ramadori G (2007b) x-Irradiation in rat liver: consequent upregulation of hepcidin and downregulation of hemojuvelin and ferroportin-1 gene expression. Radiology 242:189-197

Cui YZ, Hisha H, Yang GX, Fan TX, Jin T, Li Q, Lian Z, Ikehara S (2002) Optimal protocol for total body irradiation for allogeneic bone marrow transplantation in mice. Bone Marrow Transplant 30:843-849

Czaja MJ, Geerts A, Xu J, Schmiedeberg P, Ju Y (1994) Monocyte chemoattractant protein 1 (MCP-1) expression occurs in toxic rat liver injury and human liver disease. J Leukoc Biol 55:120-126

Daly C, Rollins BJ (2003) Monocyte chemoattractant protein-1 (CCL2) in inflammatory disease and adaptive immunity: therapeutic opportunities and controversies. Microcirculation 10:247-257

Dawson LA, Guha C (2008) Hepatocellular carcinoma: radiation therapy. Cancer J 14:111-116

Demaria S, Formenti SC (2007) Sensors of ionizing radiation effects on the immunological microenvironment of cancer. Int J Radiat Biol 83:819-825

Depaolo RW, Lathan R, Rollins BJ, Karpus WJ (2005) The chemokine CCL2 is required for control of murine gastric Salmonella enterica infection. Infect Immun 73:6514-6522

Dijkstra CD, Dopp EA, Joling P, Kraal G (1985) The heterogeneity of mononuclear phagocytes in lymphoid organs: distinct macrophage subpopulations in rat recognized by monoclonal antibodies ED1, ED2 and ED3. Adv Exp Med Biol 186:409-419

Droge W (2002) Free radicals in the physiological control of cell function. Physiol Rev 82:47-95

Dudas J, Mansuroglu T, Batusic D, Ramadori G (2009) Thy-1 is expressed in myofibroblasts but not found in hepatic stellate cells following liver injury. Histochem Cell Biol 131:115-127

Dudas J, Mansuroglu T, Batusic D, Saile B, Ramadori G (2007) Thy-1 is an in vivo and in vitro marker of liver myofibroblasts. Cell Tissue Res 329:503-514

Ebbesen LS, Olesen SH, Kruhoffer M, Ingerslev J, Orntoft TF (2006) Folate deficiency induced hyperhomocysteinemia changes the expression of thrombosis-related genes. Blood Coagul Fibrinolysis $17: 293-301$

el-Khatib E, Sharplin J, Battista J (1983) The density of mouse lung in vivo following X irradiation. Int J Radiat Oncol Biol Phys 9:853-858

Emami B, Lyman J, Brown A, Coia L, Goitein M, Munzenrider JE, Shank B, Solin LJ, Wesson M (1991) Tolerance of normal tissue to therapeutic irradiation. Int J Radiat Oncol Biol Phys 21:109-122

Espina V, Wulfkuhle JD, Calvert VS, VanMeter A, Zhou W, Coukos G, Geho DH, Petricoin EF, III, Liotta LA (2006) Laser-capture microdissection. Nat Protoc 1:586-603

Etienne W, Meyer MH, Peppers J, Meyer RA, Jr. (2004) Comparison of mRNA gene expression by RT-PCR and DNA microarray. Biotechniques 36:618-6

Fehr JE, Trotter GW, Oxford JT, Hart DA (2000) Comparison of Northern blot hybridization and a reverse transcriptase-polymerase chain reaction technique for measurement of mRNA expression of metalloproteinases and matrix components in articular cartilage and synovial membrane from horses with osteoarthritis. Am J Vet Res 61:900-905 
Franchi L, Eigenbrod T, Munoz-Planillo R, Nunez G (2009) The inflammasome: a caspase-1-activation platform that regulates immune responses and disease pathogenesis. Nat Immunol 10:241-247

Frangogiannis NG, Smith CW, Entman ML (2002) The inflammatory response in myocardial infarction. Cardiovasc Res 53:31-47

Gaber MW, Naimark MD, Kiani MF (2003) Dysfunctional microvascular conducted response in irradiated normal tissue. Adv Exp Med Biol 510:391-395

Gaugler MH, Squiban C, Claraz M, Schweitzer K, Weksler B, Gourmelon P, Van der Meeren A (1998) Characterization of the response of human bone marrow endothelial cells to in vitro irradiation. Br $\mathrm{J}$ Haematol 103:980-989

Gentleman RC, Carey VJ, Bates DM, Bolstad B, Dettling M, Dudoit S, Ellis B, Gautier L, Ge Y, Gentry J, Hornik K, Hothorn T, Huber W, Iacus S, Irizarry R, Leisch F, Li C, Maechler M, Rossini AJ, Sawitzki G, Smith C, Smyth G, Tierney L, Yang JY, Zhang J (2004) Bioconductor: open software development for computational biology and bioinformatics. Genome Biol 5:R80

Geraci JP, Mariano MS (1993a) Radiation hepatology of the rat: parenchymal and nonparenchymal cell injury. Radiat Res 136:205-213

Geraci JP, Mariano MS, Jackson KL (1993b) Radiation hepatology of the rat: time-dependent recovery. Radiat Res 136:214-221

Gerard C, Rollins BJ (2001) Chemokines and disease. Nat Immunol 2:108-115

Ghezzi P, Dinarello CA, Bianchi M, Rosandich ME, Repine JE, White CW (1991) Hypoxia increases production of interleukin-1 and tumor necrosis factor by human mononuclear cells. Cytokine 3:189-194

Greco C, Catalano G, Di GA, Orecchia R (2004) Radiotherapy of liver malignancies. From whole liver irradiation to stereotactic hypofractionated radiotherapy. Tumori 90:73-79

Gregoire V (2005) Tumor control probability (TCP) and normal tissue complication probability (NTCP) in head and neck cancer. Rays 30:105-108

Grisham JW, Nopanitaya W, Compagno J, Nagel AE (1975) Scanning electron microscopy of normal rat liver: the surface structure of its cells and tissue components. Am J Anat 144:295-321

Harnacke K, Kruhoffer M, Orntoft TF, Hass R (2005) Down-modulation of poly(ADP-ribose) polymerase-1 (PARP-1) in human TUR leukemia cells restores transcriptional responsiveness for differentiation and cell cycle arrest. Eur J Cell Biol 84:885-896

Harris JG, Flower RJ, Watanabe K, Tsurufuji S, Wolitzky BA, Perretti M (1996) Relative contribution of the selectins in the neutrophil recruitment caused by the chemokine cytokine-induced neutrophil chemoattractant (CINC). Biochem Biophys Res Commun 221:692-696

Hasegawa M, Imai R, Nojima K, Sakurai H, Suzuki Y, Kawashima M, Matsuura M, Nakamura Y, Nakano T (2002) [Radiation-induced apoptosis in vivo: therapeutic significance of apoptosis in radiation therapy]. Nippon Igaku Hoshasen Gakkai Zasshi 62:535-539

Hawkins MA, Dawson LA (2006) Radiation therapy for hepatocellular carcinoma: from palliation to cure. Cancer 106:1653-1663

Helton WS, Di BA, Chari R, Schwartz M, Bruix J (2003) Treatment strategies for hepatocellular carcinoma in cirrhosis. J Gastrointest Surg 7:401-411

Hilgard P, Muller S, Hamami M, Sauerwein WS, Haberkorn U, Gerken G, Antoch G (2009) [Selective internal radiotherapy (radioembolization) and radiation therapy for HCC--current status and perspectives]. Z Gastroenterol 47:37-54

Hogaboam CM, Bone-Larson CL, Steinhauser ML, Matsukawa A, Gosling J, Boring L, Charo IF, Simpson KJ, Lukacs NW, Kunkel SL (2000) Exaggerated hepatic injury due to acetaminophen challenge in mice lacking C-C chemokine receptor 2. Am J Pathol 156:1245-1252

Hogaboam CM, Lukacs NW, Chensue SW, Strieter RM, Kunkel SL (1998) Monocyte chemoattractant protein-1 synthesis by murine lung fibroblasts modulates CD4+ T cell activation. J Immunol 160:4606-4614 
Huang DR, Wang J, Kivisakk P, Rollins BJ, Ransohoff RM (2001) Absence of monocyte chemoattractant protein 1 in mice leads to decreased local macrophage recruitment and antigen-specific $\mathrm{T}$ helper cell type 1 immune response in experimental autoimmune encephalomyelitis. J Exp Med 193:713-726

Irizarry RA, Bolstad BM, Collin F, Cope LM, Hobbs B, Speed TP (2003) Summaries of Affymetrix GeneChip probe level data. Nucleic Acids Res 31:e15

Iwatsuki S, Starzl TE, Sheahan DG, Yokoyama I, Demetris AJ, Todo S, Tzakis AG, Van Thiel DH, Carr B, Selby R, . (1991) Hepatic resection versus transplantation for hepatocellular carcinoma. Ann Surg 214:221-228

Izikson L, Klein RS, Charo IF, Weiner HL, Luster AD (2000) Resistance to experimental autoimmune encephalomyelitis in mice lacking the CC chemokine receptor (CCR)2. J Exp Med 192:1075-1080

Jaeschke H, Hasegawa T (2006) Role of neutrophils in acute inflammatory liver injury. Liver Int 26:912-919

Jaeschke H, Smith CW, Clemens MG, Ganey PE, Roth RA (1996) Mechanisms of inflammatory liver injury: adhesion molecules and cytotoxicity of neutrophils. Toxicol Appl Pharmacol 139:213-226

Jo SK, Seol MA, Park HR, Jung U, Roh C (2011) Ionising radiation triggers fat accumulation in white adipose tissue. Int J Radiat Biol 87:302-310

Ju X, Katiyar S, Wang C, Liu M, Jiao X, Li S, Zhou J, Turner J, Lisanti MP, Russell RG, Mueller SC, Ojeifo J, Chen WS, Hay N, Pestell RG (2007) Akt1 governs breast cancer progression in vivo. Proc Natl Acad Sci U S A 104:7438-7443

Kamrava M, Bernstein MB, Camphausen K, Hodge JW (2009) Combining radiation, immunotherapy, and antiangiogenesis agents in the management of cancer: the Three Musketeers or just another quixotic combination? Mol Biosyst 5:1262-1270

Karar J, Maity A (2009) Modulating the tumor microenvironment to increase radiation responsiveness. Cancer Biol Ther 8:1994-2001

Karin M, Delhase M (2000) The I kappa B kinase (IKK) and NF-kappa B: key elements of proinflammatory signalling. Semin Immunol 12:85-98

Katz J., Golden S, Wals P.A (1979) Glycogen synthesis by rat hepatocytes Biochem.J. 180: 389-402

Kiani MF, Ansari R, Gaber MW (2003) Oxygen delivery in irradiated normal tissue. J Radiat Res (Tokyo) 44:15-21

Kiani MF, Yuan H, Chen X, Smith L, Gaber MW, Goetz DJ (2002) Targeting microparticles to select tissue via radiation-induced upregulation of endothelial cell adhesion molecules. Pharm Res 19:1317-1322

Kiltie AE, Lashford LS, Gattamaneni HR (1997) Survival and late effects in medulloblastoma patients treated with craniospinal irradiation under three years old. Med Pediatr Oncol 28:348-354

Kitagawa K, Wada T, Furuichi K, Hashimoto H, Ishiwata Y, Asano M, Takeya M, Kuziel WA, Matsushima K, Mukaida N, Yokoyama H (2004) Blockade of CCR2 ameliorates progressive fibrosis in kidney. Am J Pathol 165:237-246

Knittel T, Kobold D, Saile B, Grundmann A, Neubauer K, Piscaglia F, Ramadori G (1999) Rat liver myofibroblasts and hepatic stellate cells: different cell populations of the fibroblast lineage with fibrogenic potential. Gastroenterology 117:1205-1221

Knook DL, Sleyster EC (1976) Separation of Kupffer and endothelial cells of the rat liver by centrifugal elutriation. Exp Cell Res 99:444-449

Kruse JJ, te Poele JA, Russell NS, Boersma LJ, Stewart FA (2004) Microarray analysis to identify molecular mechanisms of radiation-induced microvascular damage in normal tissues. Int J Radiat Oncol Biol Phys 58:420-426

Laemmli UK (1970) Cleavage of structural proteins during the assembly of the head of bacteriophage T4.. Nature 227:680-685

Lawrence TS, Robertson JM, Anscher MS, Jirtle RL, Ensminger WD, Fajardo LF (1995) Hepatic toxicity resulting from cancer treatment. Int J Radiat Oncol Biol Phys 31:1237-1248 
Le Y, Zhou Y, Iribarren P, Wang J (2004) Chemokines and chemokine receptors: their manifold roles in homeostasis and disease. Cell Mol Immunol 1:95-104

Leach JK, Van TG, Lin PS, Schmidt-Ullrich R, Mikkelsen RB (2001) Ionizing radiation-induced, mitochondriadependent generation of reactive oxygen/nitrogen. Cancer Res 61:3894-3901

Lee JC, Krochak R, Blouin A, Kanterakis S, Chatterjee S, Arguiri E, Vachani A, Solomides CC, Cengel KA, Christofidou-Solomidou M (2009) Dietary flaxseed prevents radiation-induced oxidative lung damage, inflammation and fibrosis in a mouse model of thoracic radiation injury. Cancer Biol Ther 8:47-53

Lentsch AB, Yoshidome H, Cheadle WG, Miller FN, Edwards MJ (1998) Chemokine involvement in hepatic ischemia/reperfusion injury in mice: roles for macrophage inflammatory protein-2 and Kupffer cells. Hepatology 27:507-512

Leung TW, Tang AM, Zee B, Yu SC, Lai PB, Lau WY, Johnson PJ (2002) Factors predicting response and survival in 149 patients with unresectable hepatocellular carcinoma treated by combination cisplatin, interferon-alpha, doxorubicin and 5-fluorouracil chemotherapy. Cancer 94:421-427

Li YQ, Ballinger JR, Nordal RA, Su ZF, Wong CS (2001) Hypoxia in radiation-induced blood-spinal cord barrier breakdown. Cancer Res 61:3348-3354

Lin BR, Yu CJ, Chen WC, Lee HS, Chang HM, Lee YC, Chien CT, Chen CF (2009) Green tea extract supplement reduces D-galactosamine-induced acute liver injury by inhibition of apoptotic and proinflammatory signaling. J Biomed Sci 16:35

Lin M, Carlson E, Diaconu E, Pearlman E (2007) CXCL1/KC and CXCL5/LIX are selectively produced by corneal fibroblasts and mediate neutrophil infiltration to the corneal stroma in LPS keratitis. J Leukoc Biol 81:786-792

Lin M (2008) Facilitation of Neutrophil Migration Through the Corneal Stroma During Keratitis - MMP-8 and Chemokines. PhD thesis, Case Western Reserve University, Cleveland, Ohio, USA

Lo CH, Huang WY, Lin KT, Lin MJ, Lin TP, Jen YM (2014) Repeated stereotactic ablative radiotherapy using CyberKnife for patients with hepatocellular carcinoma. J Gastroenterol Hepatol 29:1919-1925

Luo JL, Kamata H, Karin M (2005) IKK/NF-kappaB signaling: balancing life and death--a new approach to cancer therapy. J Clin Invest 115:2625-2632

Machtay M, Scherpereel A, Santiago J, Lee J, McDonough J, Kinniry P, Arguiri E, Shuvaev VV, Sun J, Cengel K, Solomides CC, Christofidou-Solomidou M (2006) Systemic polyethylene glycol-modified (PEGylated) superoxide dismutase and catalase mixture attenuates radiation pulmonary fibrosis in the C57/b16 mouse. Radiother Oncol 81:196-205

Maj JG, Paris F, Haimovitz-Friedman A, Venkatraman E, Kolesnick R, Fuks Z (2003) Microvascular function regulates intestinal crypt response to radiation. Cancer Res 63:4338-4341

Malik IA, Moriconi F, Sheikh N, Naz N, Khan S, Dudas J, Mansuroglu T, Hess CF, Rave-Frank M, Christiansen H, Ramadori G (2010) Single-dose gamma-irradiation induces up-regulation of chemokine gene expression and recruitment of granulocytes into the portal area but not into other regions of rat hepatic tissue. Am J Pathol 176:1801-1815

Malik IA, Stange I, Martius G, Cameron S, Rave-Frank M, Hess CF, Ellenrieder V, Wolff HA (2015) Role of PECAM-1 in radiation-induced liver inflammation. J Cell Mol Med 19:2441-2452

Manco M, Marcellini M, Giannone G, Nobili V (2007) Correlation of serum TNF-alpha levels and histologic liver injury scores in pediatric nonalcoholic fatty liver disease. Am J Clin Pathol 127:954-960

Marra F (2002) Chemokines in liver inflammation and fibrosis. Front Biosci 7:d1899-d1914

Marra F, Romanelli RG, Giannini C, Failli P, Pastacaldi S, Arrighi MC, Pinzani M, Laffi G, Montalto P, Gentilini P (1999) Monocyte chemotactic protein-1 as a chemoattractant for human hepatic stellate cells. Hepatology 29:140-148

Martius G, Cameron S, Rave-Frank M, Hess CF, Wolff HA, Malik IA (2015) The Anti-TNF-alpha Antibody Infliximab Inhibits the Expression of Fat-Transporter-Protein FAT/CD36 in a Selective HepaticRadiation Mouse Model. Int J Mol Sci 16:4682-4697 
Masunaga S, Ono K (2002) Significance of the response of quiescent cell populations within solid tumors in cancer therapy. J Radiat Res (Tokyo) 43:11-25

Mathurin P, Rixe O, Carbonell N, Bernard B, Cluzel P, Bellin MF, Khayat D, Opolon P, Poynard T (1998) Review article: Overview of medical treatments in unresectable hepatocellular carcinoma--an impossible meta-analysis? Aliment Pharmacol Ther 12:111-126

Matsukawa A, Hogaboam CM, Lukacs NW, Lincoln PM, Strieter RM, Kunkel SL (1999) Endogenous monocyte chemoattractant protein-1 (MCP-1) protects mice in a model of acute septic peritonitis: cross-talk between MCP-1 and leukotriene B4. J Immunol 163:6148-6154

Matsumura S, Wang B, Kawashima N, Braunstein S, Badura M, Cameron TO, Babb JS, Schneider RJ, Formenti SC, Dustin ML, Demaria S (2008) Radiation-induced CXCL16 release by breast cancer cells attracts effector T cells. J Immunol 181:3099-3107

Mbeunkui F, Johann DJ, Jr. (2009) Cancer and the tumor microenvironment: a review of an essential relationship. Cancer Chemother Pharmacol 63:571-582

Mizumoto K, Qian LW, Zhang L, Nagai E, Kura S, Tanaka M (2002) A nitroimidazole derivative, PR-350, enhances the killing of pancreatic cancer cells exposed to high-dose irradiation under hypoxia. J Radiat Res (Tokyo) 43:43-51

Moeller BJ, Cao Y, Li CY, Dewhirst MW (2004) Radiation activates HIF-1 to regulate vascular radiosensitivity in tumors: role of reoxygenation, free radicals, and stress granules. Cancer Cell 5:429-441

Morgan WF, Sowa MB (2005) Effects of ionizing radiation in nonirradiated cells. Proc Natl Acad Sci U S A $102: 14127-14128$

Moriconi F, Christiansen H, Raddatz D, Dudas J, Hermann RM, Rave-Frank M, Sheikh N, Saile B, Hess CF, Ramadori G (2008) Effect of radiation on gene expression of rat liver chemokines: in vivo and in vitro studies. Radiat Res 169:162-169

Moriconi F, Malik I, Ahmad G, Dudas J, Rave-Frank M, Vorwerk H, Hille A, Hess CF, Ramadori G, Christiansen H (2009) Effect of irradiation on gene expression of rat liver adhesion molecules: in vivo and in vitro studies. Strahlenther Onkol 185:460-468

Moriconi F, Malik IA, Amanzada A, Blaschke M, Raddatz D, Khan S, Ramadori G (2012) The anti-TNF-alpha antibody infliximab indirectly regulates PECAM-1 gene expression in two models of in vitro blood cell activation. Lab Invest 92:166-177

Morimoto H, Hirose M, Takahashi M, Kawaguchi M, Ise H, Kolattukudy PE, Yamada M, Ikeda U (2008) MCP1 induces cardioprotection against ischaemia/reperfusion injury: role of reactive oxygen species. Cardiovasc Res 78:554-562

Moshage H (1997) Cytokines and the hepatic acute phase response. J Pathol 181:257-266

Neubauer K, Lindhorst A, Tron K, Ramadori G, Saile B (2008) Decrease of PECAM-1-gene-expression induced by proinflammatory cytokines IFN-gamma and IFN-alpha is reversed by TGF-beta in sinusoidal endothelial cells and hepatic mononuclear phagocytes. BMC Physiol 8:9

Ortiz-Pineda PA, Ramirez-Gomez F, Perez-Ortiz J, Gonzalez-Diaz S, Santiago-De JF, Hernandez-Pasos J, Del Valle-Avila C, Rojas-Cartagena C, Suarez-Castillo EC, Tossas K, Mendez-Merced AT, Roig-Lopez JL, Ortiz-Zuazaga H, Garcia-Arraras JE (2009) Gene expression profiling of intestinal regeneration in the sea cucumber. BMC Genomics 10:262

Park W, Lim DH, Paik SW, Koh KC, Choi MS, Park CK, Yoo BC, Lee JE, Kang MK, Park YJ, Nam HR, Ahn YC, Huh SJ (2005) Local radiotherapy for patients with unresectable hepatocellular carcinoma. Int J Radiat Oncol Biol Phys 61:1143-1150

Parkin DM (2001) Global cancer statistics in the year 2000. Lancet Oncol 2:533-543

Parola M, Robino G (2001) Oxidative stress-related molecules and liver fibrosis. J Hepatol 35:297-306

Perregaux DG, Gabel CA (1998) Post-translational processing of murine IL-1: evidence that ATP-induced release of IL-1 alpha and IL-1 beta occurs via a similar mechanism. J Immunol 160:2469-2477

Peters W, Scott HM, Chambers HF, Flynn JL, Charo IF, Ernst JD (2001) Chemokine receptor 2 serves an early and essential role in resistance to Mycobacterium tuberculosis. Proc Natl Acad Sci U S A 98:7958-7963 
Petri B, Phillipson M, Kubes P (2008) The physiology of leukocyte recruitment: an in vivo perspective. J Immunol 180:6439-6446

Privratsky JR, Newman DK, Newman PJ (2010) PECAM-1: conflicts of interest in inflammation. Life Sci $87: 69-82$

Proudfoot AE (2002) Chemokine receptors: multifaceted therapeutic targets. Nat Rev Immunol 2:106-115

Qesaraku B, Dudas J, Rave-Frank M, Hess CF, Ramadori G, Saile B, Christiansen H (2009) Effect of tumour necrosis factor-alpha and irradiation alone or in combination on the viability of hepatocellular and biliary adenocarcinoma cell lines in vitro. Liver Int 29:910-921

Ramadori G, Armbrust T (2001) Cytokines in the liver. Eur J Gastroenterol Hepatol 13:777-784

Ramadori G, Christ B (1999) Cytokines and the hepatic acute-phase response. Semin Liver Dis 19:141-155

Ramadori G, Moriconi F, Malik I, Dudas J (2008) Physiology and pathophysiology of liver inflammation, damage and repair. J Physiol Pharmacol 59 Suppl 1:107-117

Ramaiah SK, Jaeschke H (2007) Role of neutrophils in the pathogenesis of acute inflammatory liver injury. Toxicol Pathol 35:757-766

Rave-Frank M, Malik IA, Christiansen H, Naz N, Sultan S, Amanzada A, Blaschke M, Cameron S, Ahmad S, Hess CF, Ramadori G, Moriconi F (2013) Rat model of fractionated (2 Gy/day) 60 Gy irradiation of the liver: long-term effects. Radiat Environ Biophys 52:321-338

Reed GB, Jr., Cox AJ, Jr. (1966) The human liver after radiation injury. A form of veno-occlusive disease. Am J Pathol 48:597-611

Rej R (1989) Aminotransferases in disease. Clin Lab Med 9:667-687

Riley PA (1994) Free radicals in biology: oxidative stress and the effects of ionizing radiation. Int J Radiat Biol 65:27-33

Robbins ME and Zhao M (2007) Oxidative stress and radiation-induced late normal tissue injury. In: Qureshi GA, Parvez SH (Eds.), Oxidative Stress and Neurodegenerative Disorders. Elsevier, Amsterdam, pp. $135-164$

Rocha e Silva (1978) A brief survey of the history of inflammation. Agents Actions 8:45-49

Rogler G, Andus T (1998) Cytokines in inflammatory bowel disease. World J Surg 22:382-389

Rossi DL, Hurst SD, Xu Y, Wang W, Menon S, Coffman RL, Zlotnik A (1999) Lungkine, a novel CXC chemokine, specifically expressed by lung bronchoepithelial cells. J Immunol 162:5490-5497

Roxin T, Sighetea E, Bujar H (1978) The effects of ultrafractionated X-ray therapy in malignant lymphomas. Med Interne 16:285-289

Sakata K, Someya M, Matsumoto Y, Hareyama M (2007) Ability to repair DNA double-strand breaks related to cancer susceptibility and radiosensitivity. Radiat Med 25:433-438

Schecter AD, Berman AB, Yi L, Ma H, Daly CM, Soejima K, Rollins BJ, Charo IF, Taubman MB (2004) MCP1-dependent signaling in CCR2(-/-) aortic smooth muscle cells. J Leukoc Biol 75:1079-1085

Seglen PO (1972) Preparation of rat liver cells. I. Effect of Ca 2+ on enzymatic dispersion of isolated, perfused liver. Exp Cell Res 74:450-454

Semenza GL, Agani F, Feldser D, Iyer N, Kotch L, Laughner E, Yu A (2000) Hypoxia, HIF-1, and the pathophysiology of common human diseases. Adv Exp Med Biol 475:123-130

Sempoux C, Horsmans Y, Geubel A, Fraikin J, Van Beers BE, Gigot JF, Lerut J, Rahier J (1997) Severe radiation-induced liver disease following localized radiation therapy for biliopancreatic carcinoma: activation of hepatic stellate cells as an early event. Hepatology 26:128-134

Seong J, Park HC, Han KH, Chon CY, Chu SS, Kim GE, Suh CO (2003) Clinical results of 3-dimensional conformal radiotherapy combined with transarterial chemoembolization for hepatocellular carcinoma in the cirrhotic patients. Hepatol Res 27:30-35 
Sharplin J, Franko AJ (1989) A quantitative histological study of strain-dependent differences in the effects of irradiation on mouse lung during the intermediate and late phases. Radiat Res 119:15-31

Sheikh N (2006) Regulation of Gene Expression of Hepcidin and of Other Proteins of the Iron Metabolism in the Liver and in the Extrahepatic Tissues: In Vivo and In Vitro Studies in Different Rat Models. PhD thesis, University of Göttingen, Germany

Sheikh N, Tron K, Dudas J, Ramadori G (2006a) Cytokine-induced neutrophil chemoattractant-1 is released by the noninjured liver in a rat acute-phase model. Lab Invest 86:800-814

Shim SJ, Seong J, Lee IJ, Han KH, Chon CY, Ahn SH (2007) Radiation-induced hepatic toxicity after radiotherapy combined with chemotherapy for hepatocellular carcinoma. Hepatol Res 37:906-913

Smith PK, Krohn RI, Hermanson GT, Mallia AK, Gartner FH, Provenzano MD, Fujimoto EK, Goeke NM, Olson BJ, Klenk DC (1985) Measurement of protein using bicinchoninic acid. Anal Biochem 150:76-85

Smyth GK (2004) Linear models and empirical bayes methods for assessing differential expression in microarray experiments. Stat Appl Genet Mol Biol 3:Article3

Spaeth E, Klopp A, Dembinski J, Andreeff M, Marini F (2008) Inflammation and tumor microenvironments: deflining the migratory itinerary of mesenchymal stem cells. Gene Ther 15:730-738

Stone HB, McBride WH, Coleman CN (2002) Modifying normal tissue damage postirradiation. Report of a workshop sponsored by the Radiation Research Program, National Cancer Institute, Bethesda, Maryland, September 6-8, 2000. Radiat Res 157:204-223

Streetz KL, Wustefeld T, Klein C, Manns MP, Trautwein C (2001) Mediators of inflammation and acute phase response in the liver. Cell Mol Biol (Noisy -le-grand) 47:661-673

Stubbs RS, Wickremesekera SK (2004) Selective internal radiation therapy (SIRT): a new modality for treating patients with colorectal liver metastases. HPB (Oxford) 6:133-139

Sultan S, Pascucci M, Ahmad S, Malik IA, Bianchi A, Ramadori P, Ahmad G, Ramadori G (2012) LIPOCALIN-2 is a major acute-phase protein in a rat and mouse model of sterile abscess. Shock 37:191-196

Takahashi H, Nukiwa T, Yoshimura K, Quick CD, States DJ, Holmes MD, Whang-Peng J, Knutsen T, Crystal RG (1988) Structure of the human neutrophil elastase gene. J Biol Chem 263:14739-14747

Taub DD, Longo DL, Murphy WJ (1996) Human interferon-inducible protein-10 induces mononuclear cell infiltration in mice and promotes the migration of human $\mathrm{T}$ lymphocytes into the peripheral tissues and human peripheral blood lymphocytes-SCID mice. Blood 87:1423-1431

Tello K, Christiansen H, Gurleyen H, Dudas J, Rave-Frank M, Hess CF, Ramadori G, Saile B (2008) Irradiation leads to apoptosis of Kupffer cells by a Hsp27-dependant pathway followed by release of TNF-alpha. Radiat Environ Biophys 47:389-397

Tessier PA, Naccache PH, Clark-Lewis I, Gladue RP, Neote KS, McColl SR (1997) Chemokine networks in vivo: involvement of $\mathrm{C}-\mathrm{X}-\mathrm{C}$ and $\mathrm{C}-\mathrm{C}$ chemokines in neutrophil extravasation in vivo in response to TNF-alpha. J Immunol 159:3595-3602

Tjiu JW, Chen JS, Shun CT, Lin SJ, Liao YH, Chu CY, Tsai TF, Chiu HC, Dai YS, Inoue H, Yang PC, Kuo ML, Jee SH (2009) Tumor-associated macrophage-induced invasion and angiogenesis of human basal cell carcinoma cells by cyclooxygenase-2 induction. J Invest Dermatol 129:1016-1025

Towbin H, Staehelin T, Gordon J (1979) Electrophoretic transfer of proteins from polyacrylamide gels to nitrocellulose sheets: procedure and some applications. Proc Natl Acad Sci U S A 76:4350-4354

Tron K (2004) Molecular Mechanisms of the Cytokine-dependent Induction of the Heme Oxygenase-1 Gene: In Vivo and In Vitro Studies. PhD thesis, University of Göttingen, Germany

Tsou CL, Peters W, Si Y, Slaymaker S, Aslanian AM, Weisberg SP, Mack M, Charo IF (2007) Critical roles for CCR2 and MCP-3 in monocyte mobilization from bone marrow and recruitment to inflammatory sites. J Clin Invest 117:902-909

Turler A, Schwarz NT, Turler E, Kalff JC, Bauer AJ (2002) MCP-1 causes leukocyte recruitment and subsequently endotoxemic ileus in rat. Am J Physiol Gastrointest Liver Physiol 282:G145-G155

Ueda T, Sakabe T, Oka M, Maeda Y, Nishida M, Murakami F, Maekawa T (2000) Levels of 
interleukin(IL)-6, IL-8, and IL-1 receptor antagonist in the hepatic vein following liver surgery.

Hepatogastroenterology 47:1048-1051

Vercesi AE, Kowaltowski AJ, Grijalba MT, Meinicke AR, Castilho RF (1997) The role of reactive oxygen species in mitochondrial permeability transition. Biosci Rep 17:43-52

Vujaskovic Z, Anscher MS, Feng QF, Rabbani ZN, Amin K, Samulski TS, Dewhirst MW, Haroon ZA (2001) Radiation-induced hypoxia may perpetuate late normal tissue injury. Int J Radiat Oncol Biol Phys 50:851-855

Wang S, Quadri SM, Tang XZ, Stephens LC, Lollo CP, Bartholomew RM, Vriesendorp HM (1995) Liver toxicity induced by combined external-beam irradiation and radioimmunoglobulin therapy. Radiat Res 141:294-302

Witz IP (2009) The tumor microenvironment: the making of a paradigm. Cancer Microenviron 2 Suppl 1:9-17

Wolpe SD, Sherry B, Juers D, Davatelis G, Yurt RW, Cerami A (1989) Identification and characterization of macrophage inflammatory protein 2. Proc Natl Acad Sci U S A 86:612-616

Woo CW, Siow YL, O K (2008) Homocysteine induces monocyte chemoattractant protein-1 expression in hepatocytes mediated via activator protein-1 activation. J Biol Chem 283:1282-1292

Wouters A, Pauwels B, Lardon F, Vermorken JB (2007) Review: implications of in vitro research on the effect of radiotherapy and chemotherapy under hypoxic conditions. Oncologist 12:690-712

Yoshimura T, Takahashi M (2007) IFN-gamma-mediated survival enables human neutrophils to produce MCP1/CCL2 in response to activation by TLR ligands. J Immunol 179:1942-1949

Zamara E, Galastri S, Aleffi S, Petrai I, Aragno M, Mastrocola R, Novo E, Bertolani C, Milani S, Vizzutti F, Vercelli A, Pinzani M, Laffi G, LaVilla G, Parola M, Marra F (2007) Prevention of severe toxic liver injury and oxidative stress in MCP-1-deficient mice. J Hepatol 46:230-238

Zibrova D (2004) Adenovirus-mediated Gene Transfer of FK506-binding Proteins FKBP12.6 and FKBP12 in Failing and Non-failing Rabbit Ventricular Myocytes. PhD thesis, University of Göttingen, Germany

Zingg D, Riesterer O, Fabbro D, Glanzmann C, Bodis S, Pruschy M (2004) Differential activation of the phosphatidylinositol 3'-kinase/Akt survival pathway by ionizing radiation in tumor and primary endothelial cells. Cancer Res 64:5398-5406 


\section{LIST OF PUBLICATIONS}

\section{Publications:}

1. Moriconi F, Malik I, Ahmad G, Dudas J, Rave-Frank M, Vorwerk H, Hille A, Hess CF, Ramadori G, Christiansen H (2009) Effect of irradiation on gene expression of rat liver adhesion molecules: in vivo and in vitro studies. Strahlenther Onkol 185:460-468

2. Moriconi F, Ahmad G, Ramadori P, Malik I, Sheikh N, Merli M, Riggio O, Dudas J, Ramadori G (2009) Phagocytosis of gadolinium chloride or zymosan induces simultaneous upregulation of hepcidin- and downregulation of hemojuvelin- and Fpn1-gene expression in murine liver. Lab Invest 89:1252-1260

3. Mansuroglu T, Ramadori P, Dudas J, Malik I, Hammerich K, Fuzesi L, Ramadori G (2009) Expression of stem cell factor and its receptor c-Kit during the development of intrahepatic cholangiocarcinoma. Lab Invest 89:562-574

4. Ramadori G, Moriconi F, Malik I, Dudas J (2008) Physiology and pathophysiology of liver inflammation, damage and repair. J Physiol Pharmacol 59 Suppl 1:107-117

5. Malik I, Moriconi F, Sheikh N, Naz N, Khan S, Dudas J, Mansuroglu T, Hess CF, RaveFrank M, Christiansen H, Ramadori G (2010) Single-dose gamma-irradiation induces up-regulation of chemokine- gene expression and recruitment of granulocytes into the portal area but not into other regions of rat hepatic tissue. Am J Pathol. Apr; 176 (4):1801-15.

Note: Most results of the present thesis have been published in this article 


\section{ACKNOWLEDGEMENTS}

First and foremost I would like to thank Allah. In the process of putting this work together I realized how true this gift of writing is for me. You have given me the power to believe in my passion and pursue my dreams. I could never have done this without the faith I have in you, the Almighty.

Afterwards, i want to express gratitude to Professor Dr. Giuliano Ramadori for the opportunity he gave me to work in the Division of Gastroenterology and Endocrinology at the Department of Internal Medicine of Georg August University of Göttingen and for his encouragement to perform this study. I deeply appreciate the time he spent supervising and teaching me.

I express my particular gratitude to my scientific supervisor, Prof. Dr. Michael Kessel, for his outstanding supervision, support and contribution to my success. I would like to thank sincerely Professor Dr. Uwe Groß, for being official reviewer of my thesis. Their constructive criticism and suggestions are highly appreciated.

My sincere thanks to Dr. Hans Christiansen and Miss Margret Rave-Fränk from Department of Radiation Oncology for their cooperation, practical advices and the time they spent for time to time discussion about.

I would like to thank my colleagues from the institute specially Dr. Martina Blaschke for her great peice of help in thesis correction during last days and Dr. Nadeem Sheikh, Dr. Joszef Dudas, Dr. Tümen Mansuroglu and Dr. Silke Cameron for their valuable suggestions to my work. I am also grateful to the technical personnel of the department, especially S. Heyroth, A. Herbst and E. Neumann for their significant help during these years. I would also like to thank all of my friends who shared the ups and downs during my stay in Germany.

I am very grateful for all other colleagues Naila Naz, Sajjad Khan, Gesa Martius and Sadaf Sultan in the department for providing me very nice and friendly working atmosphere and technical assistance.

Mentioning some people by name is not as important as is to admire and admit their valuable role in keeping me mobile and energetic.

Last but not the least; I must not miss the opportunity to acknowledge the role of my parents and family in my success. Without their support it would have been a dream which became a reality today and I finished my Ph.D.

Thanks Mom and Dad. 


\section{CURRICULUM VITAE}

Family Name

First name

Date of birth

Place of birth

Citizenship

$1995-1997$

$1997-2001$

2003-2005

Feb. 2007-Dec. 2009
Malik

Ihtzaz-Ahmed

02.07 .1978

Gujrat, Pakistan

Pakistani

College Education

Lahore, Pakistan

Degree: Bachelors of Science (Hons.)

Agriculture faculty, AJK University, Pakistan

Degree: Master of Science (Agriculture)

Supervisor: Prof. Dr. Petr Karlovsky

Georg-August University, Göttingen

Degree: Ph.D. in biology

Department of Gastroenterology and Endocrinology of

University Hospital, Georg-August University, Göttingen

Project leader: Prof. Dr. Giuliano Ramadori

Title: "Effect of Radiation on Leukocytes Migration and Gene expression of Rat Liver Chemokines: In-vivo and In-vitro Studies" 